EVALUACIÓN DE LA EFICIENCIA DE LA RED PÚBLICA HOSPITALARIA DE BOGOTÁ D.C. 2002-2015

LUIS FERNANDO VANEGAS RIVERA

UNIVERSIDAD SANTO TOMAS

MAESTRIA EN CIENCIAS ECONOMICAS

BOGOTA D.C

2018 
EVALUACIÓN DE LA EFICIENCIA DE LA RED PÚBLICA HOSPITALARIA DE BOGOTÁ D.C. 2002-2015

LUIS FERNANDO VANEGAS RIVERA

Trabajo de grado presentado para optar al título de Magister en Ciencias Económicas

UNIVERSIDAD SANTO TOMAS

MAESTRIA EN CIENCIAS ECONOMICAS

BOGOTA D.C

2018 


\section{Contenido}

RESUMEN 9

INTRODUCCIÓN 10

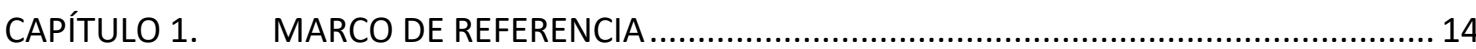

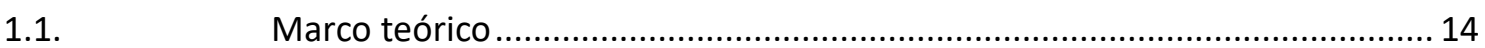

1.1.1. Determinantes de la eficiencia económica desde el punto de vista neoinstitucional 18

1.1.2. Pérdida de eficiencia económica y elasticidad............................................... 19

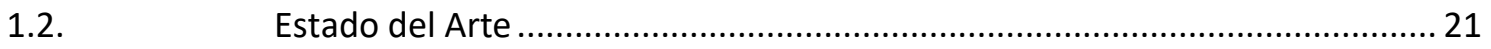

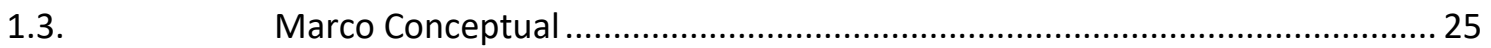

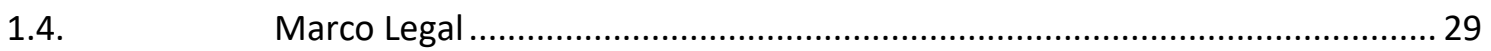

1.5. ANALISIS DEL ENTORNO DE LA RED HOSPITALARIA DE BOGOTA, EN TERMINOS ADMINISTRATIVOS, FINANCIEROS Y GESTIÓN HOSPITALARIA ................................. 34

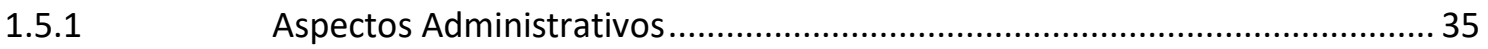

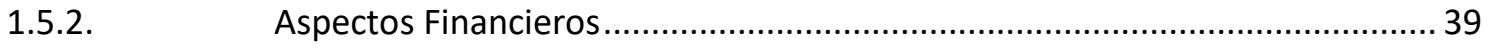

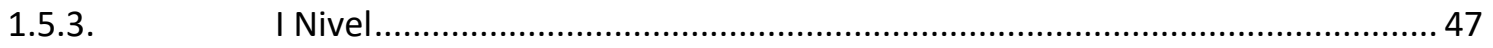

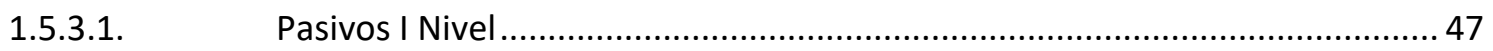

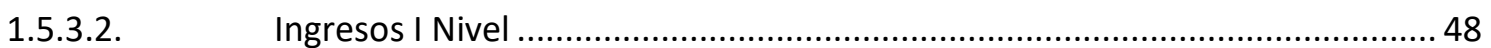

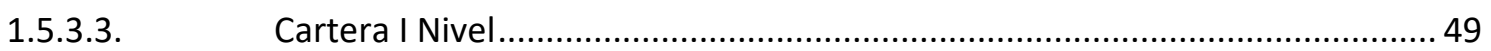

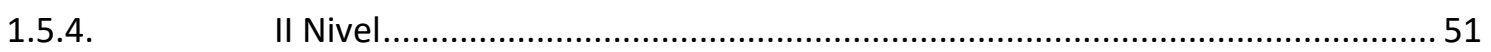

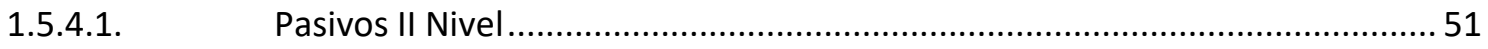

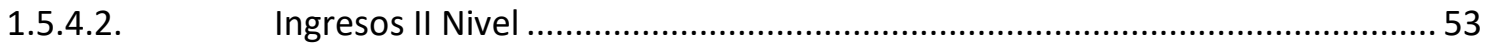

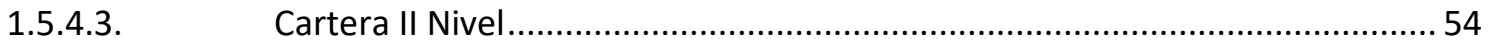

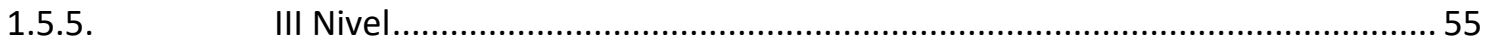

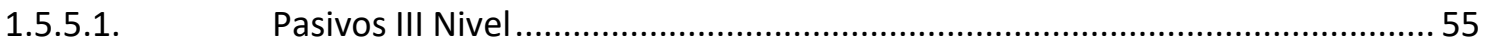

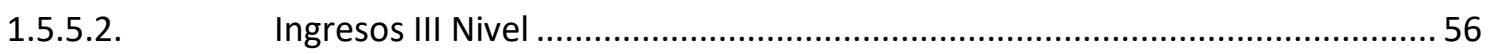

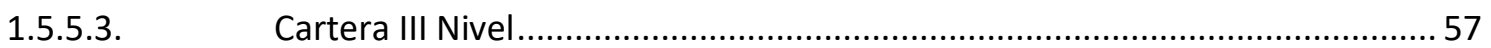

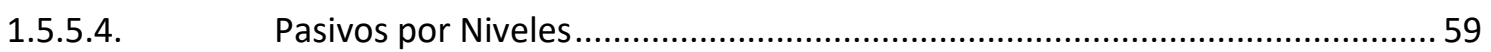

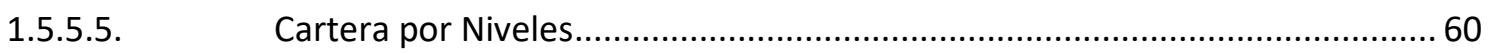

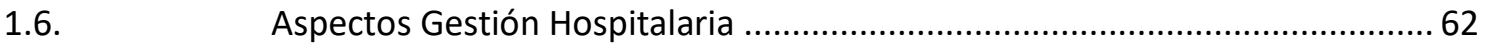

CAPÍTULO 2. EVALUACIÓN DE EFICIENCIA DE LA RED PÚBLICA HOSPITALARIA DE BOGOTÁ 72

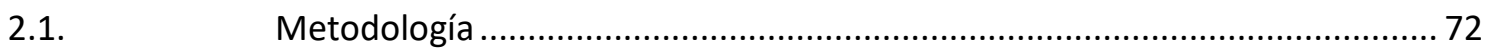

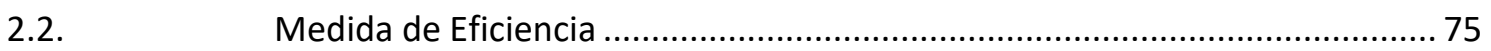

2.3. Criterios de Inclusión, exclusión y clasificación de resultados .......................... 76 


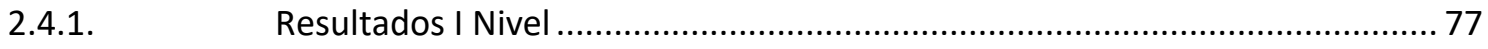

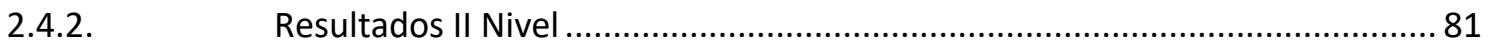

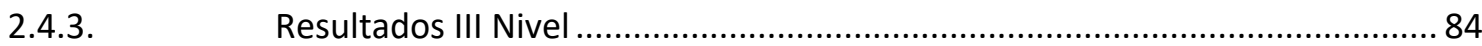

2.4.4. Propuesta de intervención para mejorar la eficiencia y productividad en la prestación de los servicios de la red hospitalaria de Bogotá, a partir de los resultados logrados en la evaluación de la eficiencia de la red pública hospitalaria .................................................... 87

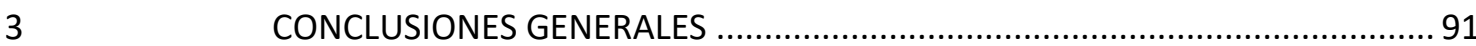

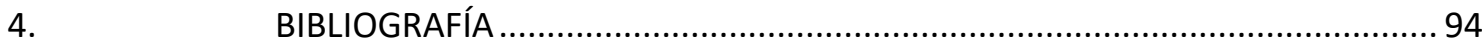

ANEXOS 98 
TABLA DE FIGURAS

Figura 1 Caja de Edgeword de eficiencia de producción ......................................................... 20

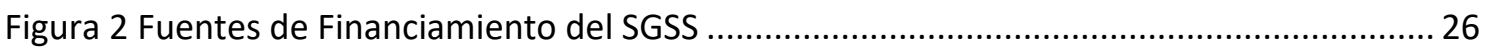

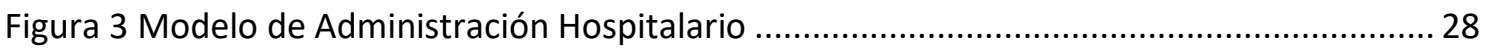

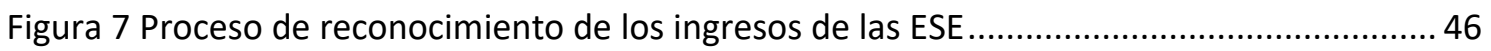

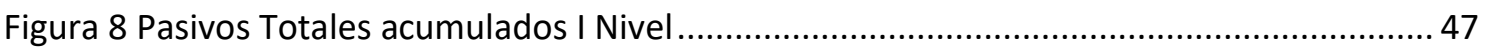

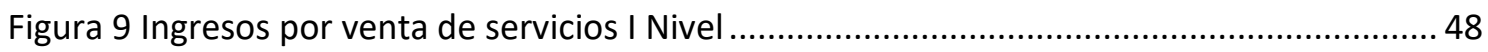

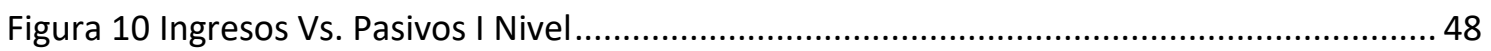

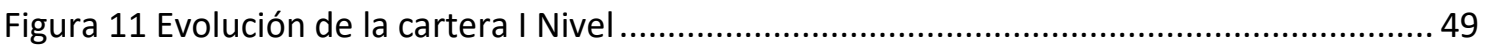

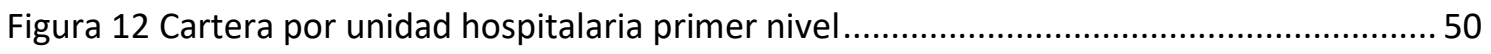

Figura 13 Evolución de los pasivos del II Nivel de Atención...................................................... 51

Figura 14 evolución de los pasivos por unidad hospitalaria de II Nivel ...................................... 52

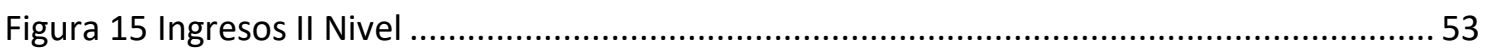

Figura 16 Evolución de la Cartera consolidada de los Hospitales de II Nivel ............................... 54

Figura 17 Evolución de la Cartera de los hospitales de II Nivel...................................................5 55

Figura 18 evolución de los pasivos consolidados de los hospitales de III Nivel ........................... 56

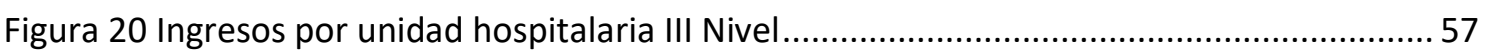

Figura 21 Evolución de la cartera consolidada de los hospitales de III nivel ..............................5 57

Figura 22 Evolución de la cartera por unidad hospitalaria de III Nivel.....................................58

Figura 23 Evolución de los pasivos consolidados por niveles de atención ................................ 59

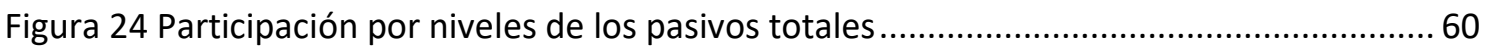

Figura 25 Evolución de la cartera total de los hospitales de Bogotá ........................................60

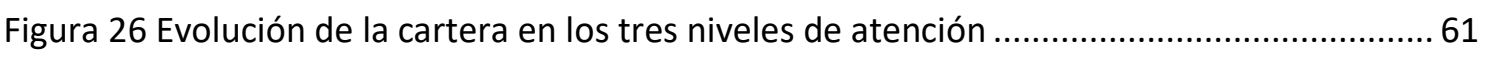

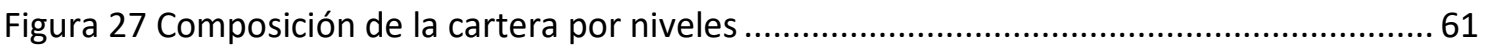

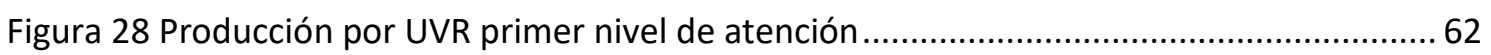

Figura 29 Producción equivalente UVR por Hospital primer nivel............................................63

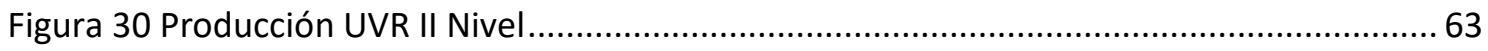

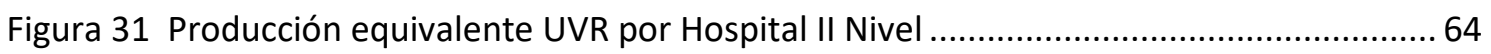

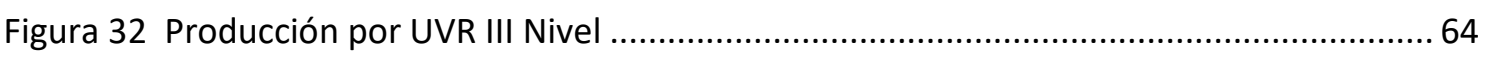

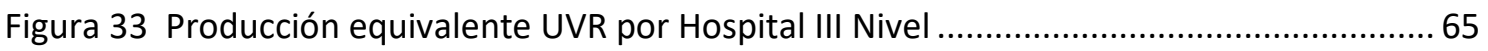

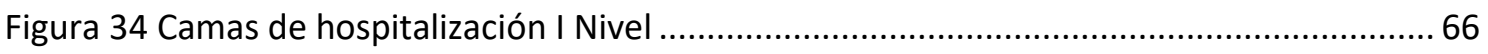

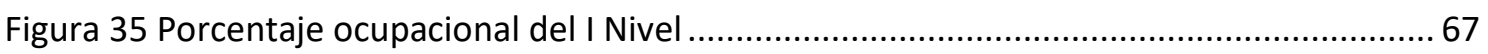

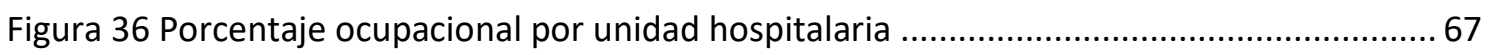

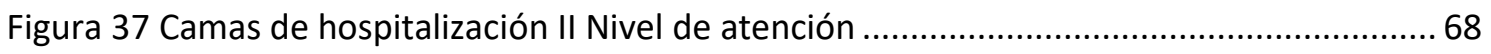

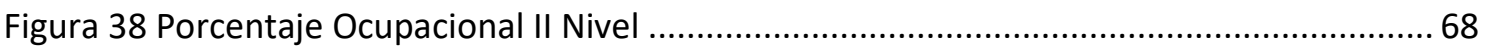

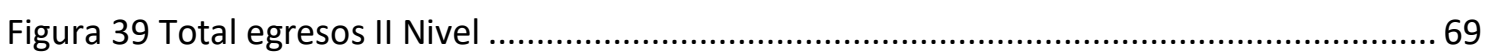

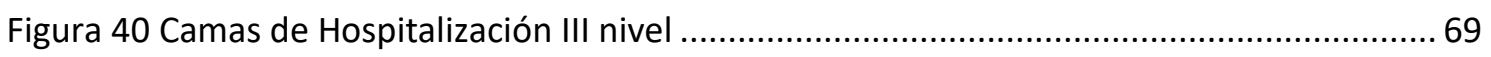

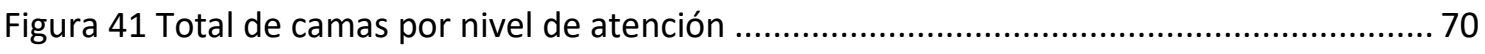

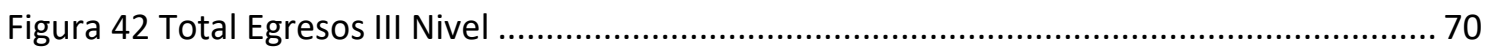

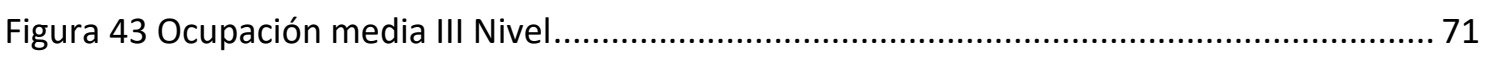

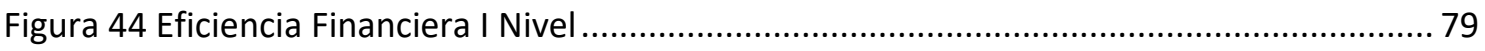

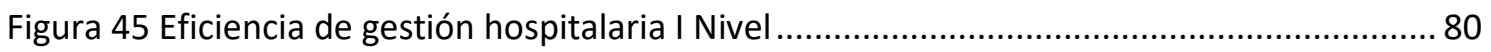

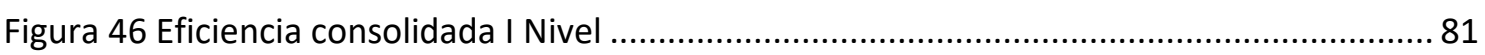

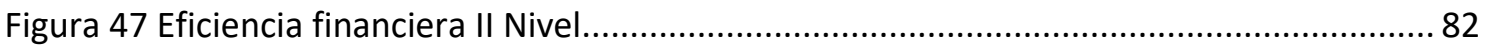

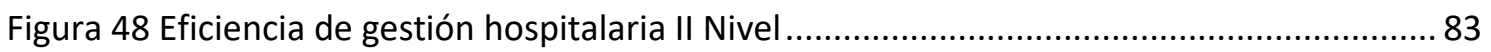




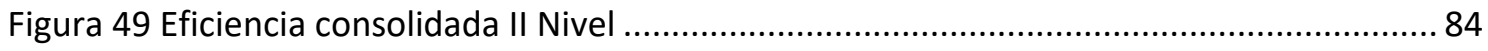

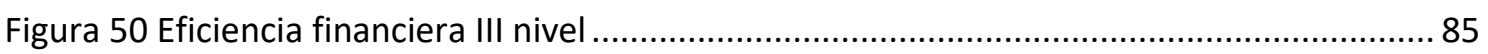

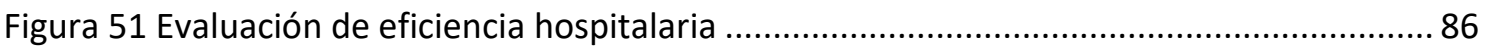

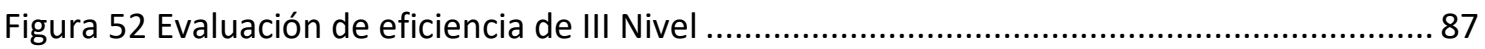




\section{TABLAS DE INFORMACIÓN}

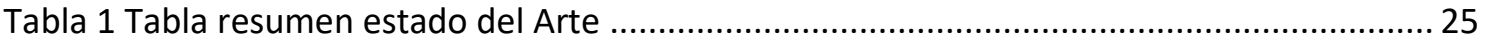

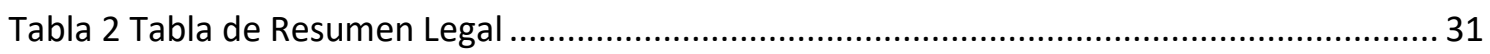

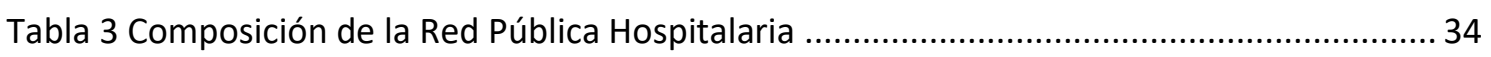

Tabla 4 Composición de la Red Pública de Hospitales por Niveles de Atención.......................... 36

Tabla 5 Resumen de Resoluciones de calificación de riesgo de las ESE Ministerio de Salud ...... 38

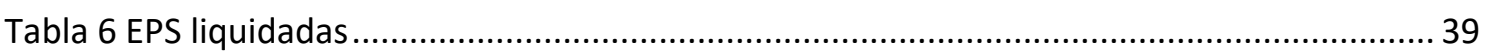

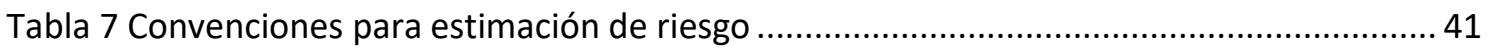

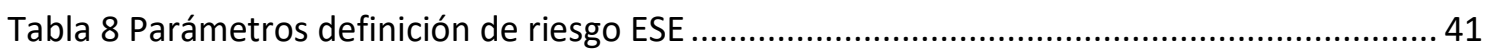

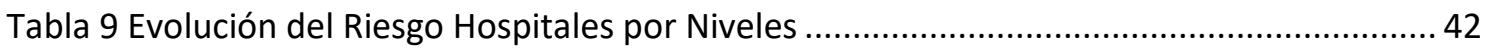

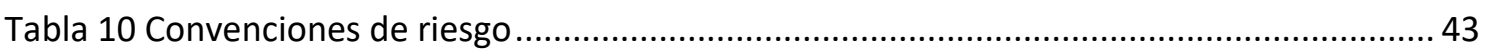

Tabla 11 Evolución indicador 9 de la Resolución 710 de 2012 para I Nivel de Atención............. 44

Tabla 12 Evolución indicador 9 de la Resolución 710 de 2012 para II Nivel de Atención............ 45

Tabla 13 Evolución indicador 9 de la Resolución 710 de 2012 para III Nivel de Atención.......... 45

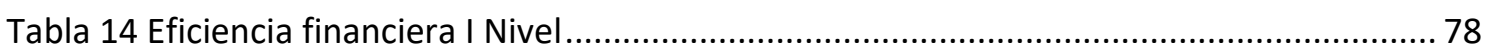

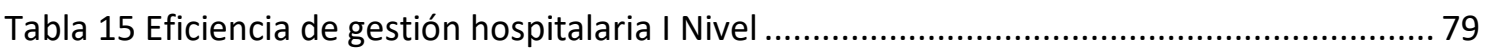

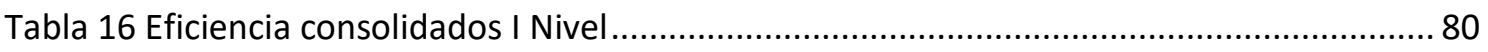

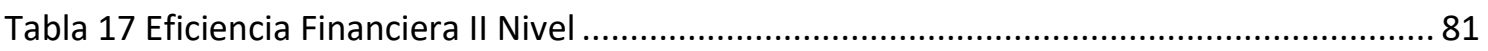

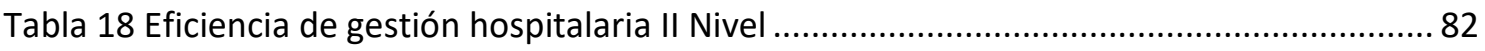

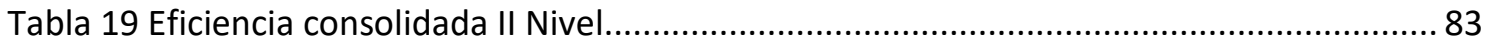

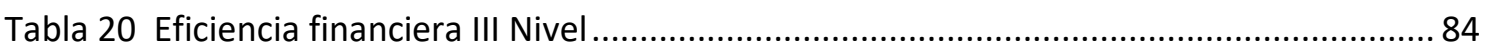

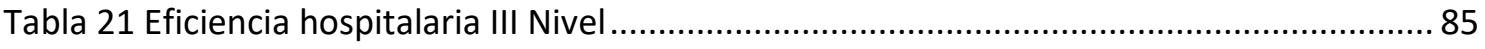

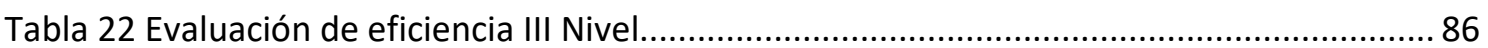

Anexos

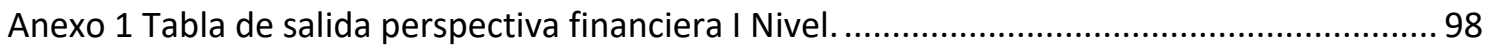

Anexo 2 Tabla de salidas perspectiva científica I Nivel .............................................................. 99

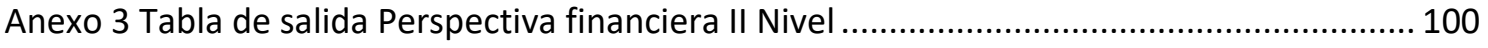

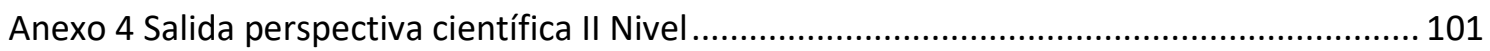

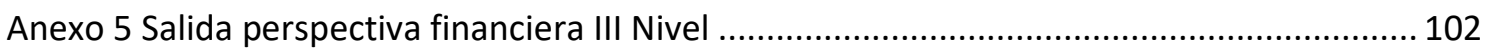

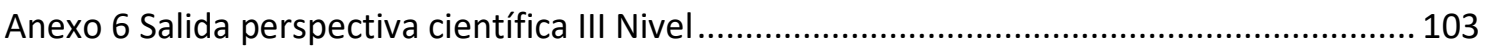


Dedicado al amor más grande del universo Alba Lucy Garzón V, madre de mis hijos e inagotable fuente de inspiración de mi crecimiento personal 


\section{RESUMEN}

La evaluación de la eficiencia de la red pública hospitalaria de Bogotá D.C. 2002-2015 se desarrolló con el fin de revisar el comportamiento de la red hospitalaria de Bogotá por niveles de atención respecto al mejor uso de los recursos disponibles en un mercado regulado. La metodología no paramétrica escogida fue el análisis envolvente de datos, una metodología en la que se revisa el comportamiento de las finanzas de las ESE y los resultados de gestión hospitalaria.

Los resultados obtenidos advierten que los hospitales de II y III nivel de complejidad presentan una pérdida sistemática de eficiencia el periodo objeto de estudio; mientras que para el I nivel los resultados no son concluyentes.

Las limitaciones frente a las conclusiones radican fundamentalmente en que la organización de la red pública hospitalaria cambió su estructura en 2016 y las recomendaciones del estudio deben ser adaptadas.

Las diferencias respecto de otros estudios de optimización radican fundamentalmente en que se tomaron aspectos financieros y también hospitalarios. 


\section{INTRODUCCIÓN}

El uso racional de los recursos garantiza que estos alcancen para hacer más y mejores cosas; en Colombia el Sistema General de Seguridad Social en Salud se articula como un mercado regulado (Flórez Pinilla, 2010); el propósito de regulación se encamina a favorecer la construcción de una sociedad equitativa con una demanda creciente por servicios de salud, que exige del sistema respuestas eficaces.

La provisión de servicios por parte de las empresas públicas es una práctica común, se trata fundamentalmente de que el Estado proporcione servicios de interés público en concordancia con la necesidad de ejercer un control político y de garantizar el acceso, y en el caso del objeto de estudio del presente documento, acceso a la salud.

Se exige sostenibilidad a las Empresas Sociales del Estado, es decir que cubran los gastos de funcionamiento y operación a cambio de que las inversiones de capital sean cubiertas por el Estado; no obstante que existe una fuerte regulación por medio de la cual se busca el control del sistema general de seguridad social en salud y de sus diferentes componentes incluidas por supuesto las Instituciones Prestadoras de Salud, que en la red pública hospitalaria funcionan como empresas sociales del Estado.

El decreto 1876 de 1994 define la naturaleza jurídica de las Empresas Sociales del Estado y expresa que "constituyen una categoría especial de entidad pública, descentralizada, con personería jurídica, patrimonio propio y autonomía administrativa, creadas o reorganizadas por ley o por las asambleas o concejos."

Las Empresas Sociales del Estado se han convertido en un instrumento de desarrollo de las relaciones Estado-Sociedad principalmente por la evolución que ha tenido el concepto de salud. La salud fue considerada, en los grandes cambios de 1991, como un servicio público; la constitución del 91 implementó un instrumento para proteger los Derechos constitucionales que fue la tutela. La sociedad reclamó mediante este mecanismo que el derecho a la salud se reconociera como un derecho conexo con a la vida (que se considera un derecho de primera generación o fundamental) y finalmente la Corte Constitucional mediante sentencia T-016/07 y sentencias sucesivas, es decir crea jurisprudencia sobre la condición de la salud como derecho fundamental, situación que se refrenda mediante la Ley Estatutaria 1751 de 2015 "por medio de la cual se regula el derecho fundamental a la salud y se dictan otras disposiciones"

La Política Nacional de Prestación de Servicios de Salud con las cual se determinaron los lineamientos estratégicos sobre los cuales se debe regir la prestación de los servicios contempla la garantía al acceso a los servicios de salud, el mejoramiento permanente de la calidad de la atención en salud, y la búsqueda y generación de eficiencia en la prestación de los servicios de salud a la población colombiana (Departamento Nacional de Planeación, 2014). 
El Plan Nacional de Desarrollo - PND 2014-2018, en el Capítulo VI Movilidad Social, Objetivo 2 Mejorar las condiciones de salud de la población contempla la necesidad de que las IPS funcionen en condiciones de eficiencia.

Plan Decenal de Salud Pública - PDSP 2012 -2021 que refleja la forma en que el gobierno nacional se alinea con la Organización Mundial de la Salud - OMS y con la Organización Panamericana de la Salud - OPS considera que la "evaluación" es la "valoración o estimación sistemática y objetiva de una intervención en curso o terminada, comprendiendo su diseño, ejecución y resultados. Lo que se busca es determinar la relevancia y satisfacción de objetivos, la eficiencia del desarrollo, su efectividad, impacto y sostenibilidad. La evaluación debe proporcionar información creíble y útil, permitiendo incorporar las lecciones aprendidas de todos los actores involucrados" (DANE, 2009).

Resultado de la propuesta de la política pública en materia de salud, de la propuesta del plan de Desarrollo "Todos por un nuevo país" y del Plan Decenal de Salud Pública surge la necesidad de preguntar ¿Cómo ha sido el comportamiento en materia de eficiencia de la red pública hospitalaria de Bogotá 2002-2015? Debido a que en los lineamientos no existen parámetros sobre la forma en que se debe medir la eficiencia más allá de las revisiones de carácter transversal que se logra con los indicadores de desempeño que se recogen de forma obligatoria por el decreto 2193 de 2004 como se explicará mas adelante.

Las necesidades es establecer condiciones de eficiencia y de evaluar un periodo razonablemente amplio para la red pública hospitalaria de Bogotá se concatenan en el planteamiento del objetivo principal del presente documento; evaluar la eficiencia de la red pública hospitalaria de Bogotá 2002-2015, que es un periodo en el que no se presentaron grandes cambios en la conformación de la red, la cual estuvo constituida por 22 Empresas Sociales hasta 2016 donde se configuraron 4 redes.

La evaluación de eficiencia de la Red Pública hospitalaria de Bogotá no presenta antecedentes recientes, razón por la cual resulta interesante convertirla en objeto de estudio.

En 1999 con corte transversal se analizó la eficiencia de la red pública hospitalaria de Bogotá, en ese momento existían 31 hospitales públicos, mediante la metodología de análisis envolvente de datos (Data Envelopment Analysis, DEA); los autorers encontraron ineficiencias de tipo técnico y de tipo asignativo como se explica en el desarrollo de los antecedentes (Nupia \& Sánchez, 2001).

En el mundo la técnica DEA presenta un amplio conjunto de ejemplos, Japón, la India, Turquía, Inglaterra y Estados Unidos tienen trabajos de investigación en 
optimización hospitalaria, ópticas que fueron tenidas en cuenta para adelantar la presente investigación

Para cumplir con el objetivo General de la presente investigación se han propuesto dos objetivos específicos, en coherencia con el desarrollo capitular, el primero es Analizar la red pública hospitalaria de Bogotá sobre los aspectos administrativos financieros y hospitalarios en el periodo 2002-2015, esto obliga a revisar los antecedentes internacionales y nacionales mediante un estado del arte; adicionalmente para claridad de los alcances, se revisa el marco conceptual y en la parte final del Capítulo I se hace un repaso por la regulación más importante del Sistema General de Seguridad Social en Salud y de los hospitales que se configura en la revisión del Marco Legal.

El segundo objetivo específico es Establecer una estrategia de política pública encaminada a garantizar la eficiencia de la red pública hospitalaria. Se ha definido que la forma más apropiada para aproximarse al objeto de estudio es presentar los análisis descriptivos por niveles de atención y en la parte final del capítulo II y en consecuencia con los hallazgos del análisis del entorno se presenta una propuesta de intervención de la política pública que podría dar respuesta al deterioro de las finanzas de las IPS.

Como resultado de los análisis y para modificar la política pública relacionada con el Sistema General de Seguridad Social en Salud, se propone establecer una cámara de compensación entre IPS y las EPS con el fin de garantizar el flujo de recursos oportunos a las finanzas del sistema, similar a como funciona el sistema financiero colombiano.

La eficiencia fue medida por grupos homogéneos de hospitales ya que las instituciones fueron agrupadas por grado de complejidad en 3 niveles, se encontró una pérdida sistemática de la eficiencia en los hospitales de II y III nivel, mientras que los resultados para el I nivel no son concluyentes.

Como resultado de los análisis y para modificar la política pública relacionada con el Sistema General de Seguridad Social en Salud, se propone establecer una cámara de compensación entre IPS y las EPS con el fin de garantizar el flujo de recursos oportunos a las finanzas del sistema.

Los alcances de los resultados, se espera sirvan para que redes hospitalarias del país copien las mejores estrategias y desechen los comportamientos menos favorables identificados en el trabajo que se presenta a continuación, situaciones que varían con frecuencia por temas de gobernanza. 
Las limitaciones principales radican en que los resultados no se podrán aplicar de manera directa sobre la red pública de Bogotá debido a los cambios estructurales relacionados con la conformación de las redes integradas de servicios de salud. 


\title{
Evaluación de la eficiencia de la red pública hospitalaria de Bogotá D.C. 2002-2015
}

\section{CAPÍTULO 1. MARCO DE REFERENCIA}

\author{
"La planificación a largo plazo no es pensar \\ en decisiones futuras, sino en el futuro de las \\ decisiones presentes." Peter F. Drucker
}

La forma de enfrentar el problema, o la óptica de abordaje, tiene diversas perspectivas con las cuales aproximar al lector al entendimiento del entorno económico de la optimización hospitalaria por ese motivo este capítulo se divide en marco teórico, estado del arte, marco conceptual y marco legal.

En este sentido, el principal objetivo del capítulo es acotar la extensión del conocimiento para estudiar los aspectos teóricos y factuales relacionados con la optimización hospitalaria en particular de la red pública hospitalaria de Bogotá en el periodo 20022015.

\subsection{Marco teórico}

En microeconomía se estudia el comportamiento de los agentes económicos bajo el supuesto de que son racionales, por racionales se acude a los supuestos de que las preferencias de los agentes son reflexivas, transitivas y completas.

Keneth Arrow ${ }^{1}$ en 1951 publica un artículo "Social choice and the individual values" en el que propone dos criterios adicionales que son el principio de no dictadura $^{2}$ y el

\footnotetext{
${ }^{1}$ Economista estadounidense de origen judío galardonado, junto con John Hicks, con el Premio Nobel de Economía, en 1972

${ }^{2}$ Principio bajo el cual no existen individuos que determinen la ordenación de las preferencias sociales con independencia de las preferencias del resto
} 
principio de no imposición ${ }^{3}$ y explica que existe una dificultad cuando se intenta pasar de la elección individual a la elección colectiva, cuando se trata de conservar los supuestos antes descritos y concluye que no existe ninguna regla para concatenar las preferencias que cumpla con los supuestos de racionalidad esperados o requeridos, elementos que derivan en la teoría de la elección social en particular a tesis que se conoce como el teorema de la imposibilidad de Arrow (Arrow, 1951).

La cobertura del sistema de salud es también el resultado del Estado que hemos construido a partir de diversos momentos en la historia en los que se han tomado decisiones que reflejan la aceptación de la mayoría; el hombre de forma natural tiene dos razonamientos básicos, la autoconservación y la repugnancia general que siente cuando otro ser sensible perece o se le hace sufrir, motivo por el cual se espera causar a los demás el mínimo daño posible (Rousseau, 1755).

La anterior posición es el punto de partida para Rawls y es entendida en términos teóricos, es decir, que debe ser entendida como una situación ideal que refleja los óptimos racionales para el establecimiento de una sociedad con unos principios de justicia aceptados por los individuos (González, Amartya Sen y la Elección Social, 1998).

La elección social, sin embargo, ha tenido una poca influencia en la macroeconomía, la cual se sigue fundamentando en el agente representativo, con las dificultades propias que la transición entre la elección individual y la elección colectiva implican ${ }^{4}$.

De acuerdo con Jorge Iván González quien cita a Van Parijs para explicar que con Amartya Sen se ampliaron nuevamente las fronteras o limitaciones expresadas por Arrow, debido a que en palabras de Sen, se deben introducir impurezas en los sistemas de elección colectiva; lo que significa que resulta indispensable pasar por encima del purismo axiomático que limita las posibilidades de transición entre el individuo y el colectivo (González, Amartya Sen y la Elección Social, 1998).

\footnotetext{
${ }^{3}$ Principio bajo el cual la ordenación de las preferencias sociales depende de las ordenaciones individuales y no se impone por otros criterios, como pueden ser la tradición o el azar

${ }^{4}$ El teorema de la imposibilidad fue dado a conocer por primera vez por el premio Nobel de Economía Kenneth Arrow en su tesis doctoral Social Choice and Individual Values en la cual demostró que las preferencias de los agentes económicos individuales, partiendo del hecho de que son racionales deben conservarse normativas deseables de agregación en la elección, como reflexividad, transitividad y completitud; situaciones que no se cumplen salvo la existencia de un dictador.
} 
Por lo tanto, se establece una relación factual entre la construcción de la idea del Estado o del Estado ideal (con limitaciones) que podemos alcanzar en nuestra sociedad y el Sistema General de Seguridad Social en Salud.

La definición de los recursos para atender la demanda de servicios de salud son el resultado de la puesta en marcha de la política pública definida para tema; el profesor Robert J. Barro plantea una interesante discusión que se puede trasladar al sector salud, cuando afirma que el cambio de comportamiento en el gobierno se debe reflejar en el cambio de comportamiento de los privados (Barro, Blinder, \& Nordhaus, 1984), esto plantea a priori, que es posible que la intencionalidad de la política pública puede no ser correctamente interpretada por los privados, cuyo fundamento tiene correspondencia con la Teoría de la Elección Pública.

La elección pública es un marco de referencia para el análisis los problemas políticos, un ejemplo de decisión pública es la Ley Estatutaria de Salud (Ley 1751 de 2015 Por medio de la cual se regula el derecho fundamental a la salud y se dictan otras disposiciones), debido a que la estructura utilizada para aprobar la ley es representativa de la democracia y representativa del Estado que como sociedad se ha erigido, el problema de que agentes representativos (parlamentarios) escojan el modelo de salud, cuando el pueblo espera la mejor elección hace parte de las dificultades de la elección pública.

En lo anterior se fundamenta un problema entre lo que los usuarios esperan recibir como derecho a la salud y los que se construye a partir de las posibilidades de recursos que se decide en el Congreso con ponencia normalmente del Gobierno, es decir que el problema general de la economía (necesidades infinitas, recursos limitados) se traslada al sector de la salud.

La magnitud del gasto público se refleja en la oferta de bienes públicos ofrecidos, el mercado privado complementa y sustituye los vacíos que queden frente a la demanda, de tal manera que la política termina permeándolo todo.

Vickrey es un profeta cuando se anticipa en el tiempo y propone el principio de equiprobabilidad antes que Harsanyi y antes que Rawls en el concepto de posición original de 1971, cuando revela las limitaciones del análisis usual (de utilidad). Vickrey 
es meticuloso, describe su trabajo como una pintura y considera que existen limitaciones de mercado y la lógica de precios para resolver los problemas normativos (González, 2004).

La forma más acertada de distribución de recursos públicos, a partir de la visión de Vickrey, es mediante su propia analogía en la que plantea una suposición en la que algunas naves parten de manera simultánea del puerto de New York a diferentes islas, las cuales han sido dotadas de los bienes necesarios para asegurar una vida adecuada; previamente se discute en condiciones de equiprobabilidad quien va a hacer que, aun sin haber sido asignados, algo así como en los "realities" actuales, lo que asegura una propuesta de solución al problema colectivo (Vickrey W. , 1948), similar al velo de ignorancia propuesto por Rawls tiempo después.

El velo de ignorancia corresponde con una situación hipotética en la cual se acuerdan las condiciones de un contrato como derechos y obligaciones bajo "un velo de ignorancia" en las que los participantes no conocen su posición en el futuro, es como esperar que los parlamentarios piensen y decidan en la respuesta que debe dar el Estado en el caso en que llegaran a enfermarse de tal forma que esa misma respuesta fuera para la sociedad.

Para poder pensar en la satisfacción de la demanda de servicios de salud a partir de la oferta pública, es necesario establecer un imaginario de sobre la utilidad colectiva y la clase de Estado que satisface a las mayorías (si es que este mecanismo de elección social es el mejor), sin embargo, aún tenemos problemas por resolver como "taxation without representation" que se refleja en problemas asociados a la financiación de las necesidades y las asimetrías existentes frente al beneficio (pago o no) de los servicios de salud.

Resulta conveniente revisar microfundamentadamente la mejor utilización de los recursos disponibles en las IPS públicas, como solución parcial a la demanda por servicios de salud en el Distrito Capital, y al uso de los recursos disponibles, es decir que se acepta que existen problemas diversos o no diversificables que pesan en las IPS y no obstante, se considera una obligación manejar de la mejor manera posible, los recursos disponibles. 


\subsubsection{Determinantes de la eficiencia económica desde el punto de vista neoinstitucional}

Según Wiesner (1998) la eficiencia está determinada por el origen y la naturaleza de los recursos que financian un gasto dado. Cuando el financiamiento del gasto proviene de impuestos, de transferencias sin mayor condicionalidad, se dice que hay menor eficiencia que la que pueda lograrse con mediación del mercado (la mano invisible ${ }^{5}$ )

Aunque se tuviera que escoger determinantes de la eficiencia y los del mercado, se concluye que la competencia es el principal determinante toda producción y consumo. De hecho, los demás determinantes de la eficiencia tienen en común la existencia de la competencia, lo cual podría tratarse de una identidad.

En este sentido, si se describe la eficiencia hospitalaria como estrategia de mercado, se da acceso a la competencia, los agentes económicos y los agentes políticos compitiendo entre sí, los cuales inducirán a un mejor uso de los recursos.

$\mathrm{Si}$, por lo contrario, los agentes públicos son la principal modalidad de gestión y no se le da mayor margen a la competencia, entonces, en principio, habrá menos eficiencia en general. Pinzón (2003) analiza el desempeño de los hospitales públicos dentro del mercado de prestación de servicios de salud, analiza el estado de eficiencia de los hospitales de baja complejidad en un caso aplicado para Colombia, (Pinzón, 2003) llega a concluir que los mejores servicios de salud lo prestan los hospitales más grandes y el municipio donde se encuentra ubicado.

Las implicaciones de la debilidad institucional del sistema de salud agravan la debilidad del sistema; la pugna entre una regulación fuerte y un mercado débil debiera ser fácilmente solucionado, sin embargo, ni la regulación es tan fuerte para socorrer a las instituciones, ni el mercado es tan débil para que desaparezca.

\footnotetext{
${ }^{5}$ Metáfora creada en 1776 en "la riqueza de las naciones" por Adam Smith en la que se expresa que el egoísmo de un individuo por lograr su beneficio termina generando bienestar a la sociedad
} 
Esta situación de debilidad Institucional es muy importante para entender el problema de la salud; desde la perspectiva de Douglas North, los cambios tecnológicos son menos importantes que los cambios institucionales para explicar el desarrollo.

Por supuesto es importante notar que las instituciones son el conjunto de reglas, normas y principios con el que se desarrollan las relaciones entre los actores, es decir reglas claras en un sentido de historia, en un lenguaje libre de especulaciones (North, 1995).

En conclusión, la naturaleza de la eficiencia, acudiendo a la paradoja de Jevons ${ }^{6}$ implica que se pueda ampliar las coberturas de los usuarios, aunque se lleve a cuestas una percepción de insatisfacción frente a los servicios de salud, resultado de las asimetrías de información que reposan sobre la responsabilidad de los médicos y sus jueces naturales que son los usuarios siempre que no se detenga la implementación de tecnologías y las instituciones respondan a los requerimientos de la sociedad en materia de salud.

\subsubsection{Pérdida de eficiencia económica y elasticidad}

El proceso productivo hospitalario involucra la utilización de recursos diferenciados por niveles de atención, elementos que son trasformados con el objetivo de satisfacer las necesidades particulares de los usuarios; la diferenciación de los requerimientos y las posibilidades de atención definen los niveles de complejidad en concordancia con la Ley 10 de 1990 por medio de la cual se reorganiza el Sistema Nacional de Salud y se dictan otras disposiciones.

Los términos productividad y eficiencia con frecuencia se utilizan como sinónimos cuando se trata de medir el desempeño de un proceso, sin embargo, es conveniente diferenciarlos. Los métodos eficientes de producción se centran en la utilización del capital y mano de obra para producir un bien o servicio (en este caso particular servicios médicos)

\footnotetext{
${ }^{6}$ La paradoja de William Stanley Jevons, economista inglés, quien dijo que en 1865 que en la medida en la que se perfecciona la tecnología que aumenta la eficiencia del uso de un recurso, lo más probable es que aumente el consumo de dicho recurso, en vez de disminuir.
} 
Si se toma como referencia la frontera de posibilidades de producción, mostrará distintas combinaciones de bienes $\mathrm{x}$ y de y que una empresa puede producir con eficiencia, teniendo recursos fijos. Si tomamos $\mathrm{X}$ como servicios hospitalarios como atención y Y otros servicios de salud, los factores para la producción de x o de y al tiempo se mantendrían las condiciones para la eficiencia.

La figura 1 presentan una pendiente negativa de la curva de posibilidades de producción se denomina tasa de transformación del producto (TTP).

\section{Figura 1 Caja de Edgeword de eficiencia de producción}

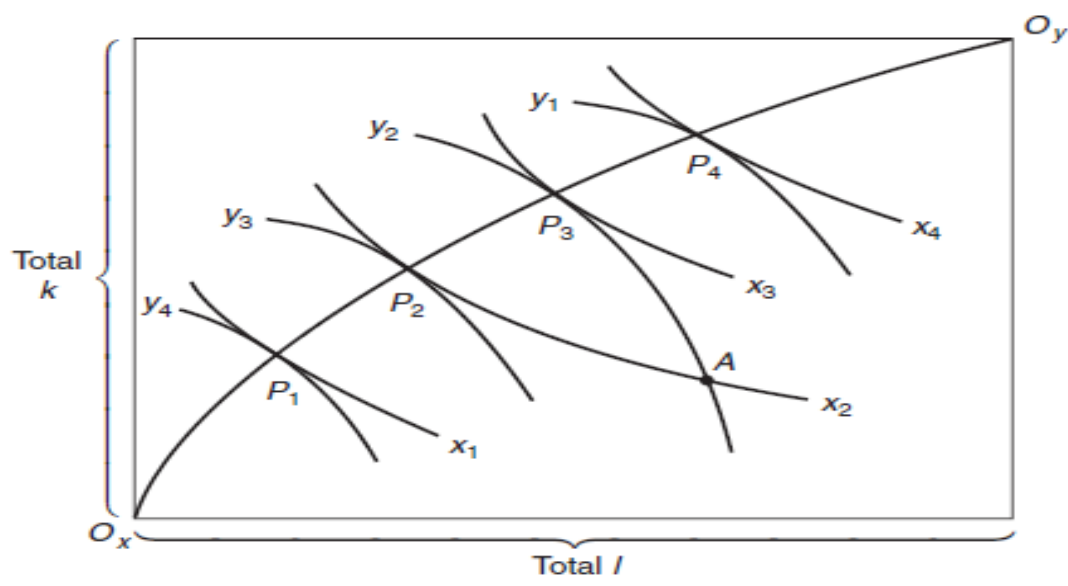

Fuente: Teoría Microeconomía, (Nicolson, 2008) pág 338

En la figura 1, las asignaciones eficientes se encuentran en P1, P2, P3 y P4, que son los precios del mercado, si vemos el servicio hospitalario como una empresa donde las isocuantas son tangentes a la curva de contrato. De acuerdo con el óptimo Paretiano, una asignación eficiente se ubicaría en P4, y es ineficiente cuando las curvas de isocuantas se cortan en el punto A.

Sin embargo, en los puntos tangentes, tal como lo muestra la figura 1, es imposible hacer mejoras inequívocas. Por ejemplo, un sistema hospitalario mejoraría su servicio o su especialización que obligaría pasar un precio de $\mathrm{P} 2$ a P3, por lo cual se estará produciendo mayor cantidad de servicios hospitalarios X. Sin embargo, un precio más alto significa barreras de acceso al servicio por parte de los usuarios. 
También se añade que se produce menos de y, por tanto P3 no será "más eficiente" que $\mathrm{P} 2$, o sea que los dos puntos son eficientes. La tangencia de las isocuantas del bien $\mathrm{x} \mathrm{y}$ del bien y implica que sus pendientes son iguales. Es decir, la Tasa Marginal de sustitución Técnica -TMST de capital por trabajo, es igual en la producción de x y de y.

Se debe tener en cuenta que la productividad se afecta por los costos hospitalarios entre los salarios de los trabajadores y el valor del capital, el cual no compensa en el crecimiento de la producción, por ende, esto es de gran influencia en el desempeño de los hospitales. Asimismo, la eficiencia (técnica), válido para el sector Hospitalario, se entiende como la capacidad de una empresa para conseguir la máxima producción a partir de un conjunto de insumos. La eficiencia técnica varía entre 0 y 1 , un valor de 1 indica que la empresa es completamente eficiente y opera en la frontera de producción; un valor menor que uno refleja que la empresa opera por debajo de la frontera y la diferencia entre 1 y el valor observado mide la ineficiencia técnica (Coelli, Estanche, Perelman, \& Trujillo, 2003). Para medir la eficiencia de las organizaciones se han utilizado diferentes métodos, tales como: índices basados en precios mediante técnicas econométricas (frontera estocástica) ${ }^{7}$ (Canay, 2001), y análisis envolvente de datos.

Para el caso colombiano, se han hecho investigaciones como la realizada por los profesores (Meza Carvajalino \& Isaza Castro, 2005) donde describe la reforma que dio lugar al Sistema General de Seguridad Social en Salud -SGSSS en Colombia (Ley 100 de 1993), sus implicaciones, sus problemas y sus virtudes. El documento deja ver las estadísticas de la población cotizante al régimen contributivo del SGSSS, Total nacional (1996-2003) y así otros cuadros y sus respectivos análisis.

\subsection{Estado del Arte}

En el Mundo la información con modelos de análisis envolvente de datos es abundante, se presentan algunos ejemplos que llamaron la atención del autor de la presente propuesta.

\footnotetext{
${ }^{7}$ Las estimaciones de fronteras de producción son una extensión de las típicas estimaciones de funciones de producción microeconómicas, basadas en la premisa de que esta función de producción representa algún tipo de ideal, la máxima cantidad de producto asequible dados los insumos De esta forma, en la práctica las fronteras de producción no son más que una regresión que se ajusta a los datos reconociendo la restricción de que todas las observaciones deben encontrarse debajo de la frontera y al menos una debe estar sobre ella
} 
En el estudio desarrollado por Kawaguchi, Tone y Tsutsui proponen evaluar el efecto político de la reforma de los hospitales municipales en Japón; se centran en la mejora de la eficiencia no sólo del hospital en sí sino también separar las organizaciones internas de un hospital. Los hospitales tienen dos organizaciones internas heterogéneas: la sección de examen médico y la sección de administración. La sección de administración lleva a cabo la administración de negocios y la sección de examen médico proporciona servicios de atención médica. Se empleó un modelo dinámico y de red de análisis envolvente de datos que hace posible estimar tanto la eficiencia de las organizaciones separadas como los cambios dinámicos de las eficiencias simultáneamente. (Kawaguchi, Tone, \& Tsutsui, 2013)

Se utiliza un modelo DEA dinámico, que permite evaluar el rendimiento de eficiencia de las unidades de toma de decisiones (DMU, por su sigla en inglés), "La función objetivo de la sección de administración es lograr una situación financiera sólida a través de insumos de mano de obra e insumos de capital. La función objetiva de la sección de exámenes médicos es proporcionar una cierta cantidad de servicio médico usando camas de hospital que son mantenidas por la administración en el mismo hospital”.

Los autores de este documento analizaron la eficiencia y el crecimiento de la productividad de una muestra representativa de hospitales portugueses de 1997 a 2004 , utilizando un enfoque que califican de innovador empleando la función de distancia direccional y el indicador de productividad de Luenberger. Para ellos la principal ventaja del enfoque es que se consideran las contracciones de entrada y las expansiones de salida. En este sentido el modelo genera un indicador de productividad que se desagrega en los componentes habituales del crecimiento de la productividad: cambio tecnológico y cambio de eficiencia.

Los resultados muestran que, en promedio, los hospitales portugueses no experimentaron crecimiento de productividad durante el período analizado. Además, la incidencia del crecimiento positivo de la productividad en Hospitales fue notablemente baja (Pestana Barros, Gomes de Menezes, Cabral Vieira, Peypoch, \& Solonandrasana , 2007).

Este estudio se centra en analizar la eficiencia hospitalaria de los hospitales gubernamentales de distrito y los hospitales subvencionados en Gujarat. El estudio intenta proporcionar una visión general de la situación general de los servicios de atención de 
salud prestados por los hospitales en el estado de Gujarat en términos de su eficiencia técnica y de asignación.

Uno de los dos objetivos de la cuestión de la eficiencia era hacer un balance del estado de los servicios de salud (en términos de eficiencia) proporcionado por los hospitales de subvención en la ayuda y los hospitales de distrito en Gujarat. La motivación detrás de abordar la cuestión de la eficiencia es proporcionar un análisis empírico de la política del gobierno para proporcionar subvenciones a las instituciones sin fines de lucro que a su vez proporcionan atención hospitalaria en el estado. El estudio aborda la cuestión de si los hospitales de subvención son relativamente más eficientes que los hospitales públicos.

Esta comparación entre los hospitales de subvención y los hospitales de distrito en términos de su eficacia ha sido de interés para muchos investigadores de países distintos de la India y no se ha llegado a un consenso en cuanto a qué categoría es más eficiente (Bhat, Bhushan Verma, \& Reuben, 2001).

Para Almula y Karsak es importante desarrollar metodologías específicas para evaluar el desempeño de los hospitales gana cada vez más importancia para la mayoría de los países, ya que los crecientes gastos en salud, el aumento de la calidad y la competencia en el sector salud requieren que los hospitales utilicen sus recursos de manera eficiente. En su trabajo se presenta un análisis envolvente de datos imprecisos (DEA) evaluando el desempeño de los hospitales estatales ubicados en 26 distritos de Estambul, la ciudad más grande de Turquía, que también figura entre las ciudades más concurridas del mundo. La metodología propuesta tiene en cuenta tanto los datos cuantitativos como los cualitativos representados como variables lingüísticas para la evaluación del desempeño. Después de realizar un análisis exhaustivo, identifican distritos eficientes e ineficientes en cuanto a la provisión de servicios de salud. (Almula Karadayi \& Karsak , 2014)

En Colombia existe evidencia de trabajos que preceden la presente propuesta, por ejemplo en marzo de 2014 Antonio Orozco presentó una aproximación regional de eficiencia y productividad mediante el indicador de Luenberger para evaluar la eficiencia y productividad de los hospitales públicos en Colombia. El análisis empírico se realizó para una muestra de 336 hospitales durante el periodo 2003-2011. Los resultados indican una caída en la productividad de los hospitales públicos colombianos, sustentada en el 
deterioro del cambio tecnológico como resultado de una escasa inversión en tecnología (Orozco Gallo, 2014).

En 2007 acudiendo al Sistema de Información Hospitalario SIHO, Norman Maldonado y Ana Tamayo, evaluaron el periodo 2002-2005 con varios resultados. Primero, se logra realizar una clasificación de hospitales diferente a la relacionada con el nivel de complejidad. En esta clasificación, se obtienen cinco grupos conformados por hospitales relativamente homogéneos al interior de cada grupo, y cada grupo con ciertas características particulares que lo diferencian de los otros.

Segundo, se obtiene una medición de eficiencia técnica comparable en el tiempo, superando estudios anteriores que no permitían tal comparación. Finalmente, se propone una metodología que supera parcialmente los problemas de medida relativa que tiene el DEA tradicional, y si bien no se puede aplicar a los datos disponibles por problemas de dimensionalidad y de pocas observaciones, si puede ser implementada en años futuros, donde se cuente con mayor número de observaciones en el tiempo (Maldonado \& Tamayo, 2007).

Pinzón en 2003 se concentró en los hospitales de baja complejidad, La investigación fue desarrollada bajo dos etapas empíricas. En la primera etapa, se recolectó información de 203 hospitales públicos de baja complejidad para el año 2001, y se estimaron los coeficientes de eficiencia técnica relativa en los hospitales de la muestra mediante la metodología Data Envelopment Analysis - DEA-. De acuerdo con los resultados, el 70\% de las instituciones analizadas operan bajo la frontera de posibilidades de producción, con un coeficiente de eficiencia en promedio de 72,2 (Pinzón, 2003).

En 1999 se revisó la eficiencia de los 31 hospitales públicos de Bogotá, utilizando información de producción e insumos de los hospitales en 1999. Mediante la aplicación de técnicas DEA (Data Envelopment Analysis), se obtuvieron medidas de eficiencia, tanto técnicas como asignativas, usando funciones de producción multiproducto y uniproducto, respectivamente, con diferentes supuestos de rendimientos a escala. Para explicar sus determinantes, se utilizan modelos de regresión lineal con técnicas de componentes principales, con la inclusión de variables exógenas que recogen aspectos de la estructura de ingresos y gastos de los hospitales, la estructura de mercado, la estructura 
laboral, la demanda de servicios y la localización geográfica. Los resultados sugieren que aproximadamente la mitad de los hospitales del distrito poseen ineficiencias de tipo técnico y la gran mayoría afronta ineficiencias de tipo asignativo. Como posibles causas de tal situación se encuentran la estructura sindical, de contratación y de subsidios a la oferta (Nupia \& Sánchez, 2001).

Tabla 1 Tabla resumen estado del Arte

\begin{tabular}{|c|c|c|c|c|c|}
\hline AÑO & LUGAR & AUTORES & OBJETO DE ESTUDIO & INSTRUMENTO & CONCLUSIONES \\
\hline 2001 & India & $\begin{array}{l}\text { Bhat, Bhushan, } \\
\text { Verna \& Reuben }\end{array}$ & $\begin{array}{l}\text { Hospitales de distrito } \\
\text { de Gujarat } \\
\text { subvencionados y NO }\end{array}$ & $\begin{array}{l}\text { DEA eficiencia } \\
\text { técnica y asignación }\end{array}$ & No concluyente \\
\hline 2004 & Portugal & $\begin{array}{l}\text { Barros, Gomes de } \\
\text { Meneses, Cabral } \\
\text { Vieira, Peypoch \& } \\
\text { Sononandrasana }\end{array}$ & $\begin{array}{l}\text { Muestra representativa } \\
\text { de hospitales } \\
\text { portugueses }\end{array}$ & $\begin{array}{l}\text { DEA Productividad, } \\
\text { cambio tecnológico } \\
\text { y crecimiento de la } \\
\text { productividad }\end{array}$ & $\begin{array}{l}\text { Los hospitales no } \\
\text { experimentaron } \\
\text { crecimiento de } \\
\text { productividad }\end{array}$ \\
\hline 2013 & Japón & $\begin{array}{l}\text { Kawaguchi, Tone y } \\
\text { Tsutsui }\end{array}$ & Hospitales Municipales & $\begin{array}{l}\text { DEA dinámico para } \\
\text { lograr una situación } \\
\text { financiera sólida }\end{array}$ & $\begin{array}{l}\text { No se encontraron } \\
\text { efectos positivos de } \\
\text { productividad a pesar } \\
\text { del aumento del } \\
\text { apoyo financiero del } \\
\text { gobierno central. }\end{array}$ \\
\hline 2014 & Turquia & $\begin{array}{l}\text { Almula, Karadayi } \\
\text { \& Karsak }\end{array}$ & $\begin{array}{l}\text { Hospitales de } 26 \\
\text { distritos de Estambul }\end{array}$ & $\begin{array}{l}\text { DEA Uso eficiente de } \\
\text { los Recursos }\end{array}$ & $\begin{array}{l}\text { Se identifican distritos } \\
\text { eficientes e } \\
\text { ineficientes }\end{array}$ \\
\hline & & & & & \\
\hline 2014 & Colombia & $\begin{array}{l}\text { Antonio Orozco } \\
\text { Gallo }\end{array}$ & 336 hospitales públicos & $\begin{array}{l}\text { DEA eficiencia y } \\
\text { productividad 2003- } \\
2011\end{array}$ & $\begin{array}{l}\text { Caída en la } \\
\text { productividad } \\
\text { sustentada en el } \\
\text { cambio tecnológico }\end{array}$ \\
\hline 2007 & Colombia & $\begin{array}{l}\text { Maldonado \& } \\
\text { Tamayo }\end{array}$ & $\begin{array}{l}\text { Hospitales por nivel de } \\
\text { complejidad }\end{array}$ & $\begin{array}{l}\text { DEA eficiencia } \\
\text { técnica }\end{array}$ & $\begin{array}{l}\text { No concluyente por } \\
\text { problemas de } \\
\text { dimensionalidad y } \\
\text { pocas observaciones } \\
\text { en el tiempo }\end{array}$ \\
\hline 2003 & Colombia & Pinzón & $\begin{array}{l}\text { Hospitales de baja } \\
\text { complejidad }\end{array}$ & $\begin{array}{l}\text { DEA eficiencia } \\
\text { técnica relativa }\end{array}$ & $\begin{array}{l}\text { 70\% operan bajo la } \\
\text { frontera de } \\
\text { posibilidades de } \\
\text { producción }\end{array}$ \\
\hline 2001 & Bogotá D.C. & Nupia \& Sánchez & $\begin{array}{l}\text { Hospitales públicos de } \\
\text { Bogotá (31) }\end{array}$ & $\begin{array}{l}\text { DEA con funciones } \\
\text { de producción } \\
\text { multiproducto }\end{array}$ & $\begin{array}{l}\text { Los resultados } \\
\text { sugieren que } \\
\text { aproximadamente la } \\
\text { mitad de los } \\
\text { hospitales del distrito } \\
\text { poseen ineficiencias } \\
\text { de tipo técnico y la } \\
\text { gran mayoría afronta } \\
\text { ineficiencias de tipo } \\
\text { asignativo }\end{array}$ \\
\hline
\end{tabular}

Fuente: Elaborado por el Autor, 2017

\subsection{Marco Conceptual}


El proceso productivo hospitalario involucra la utilización de recursos diferenciados por niveles de atención, elementos que son trasformados con el objetivo de satisfacer las necesidades particulares de los usuarios; la diferenciación de los requerimientos y las posibilidades de atención definen los niveles de complejidad que se acusan mediante el Acuerdo 20 de 1990 del Concejo de Bogotá.

Los recursos de la salud fluyen de acuerdo con las prioridades establecidas para la salud y cuenta con las siguientes fuentes de financiamiento:

\section{Figura 2 Fuentes de Financiamiento del SGSS}

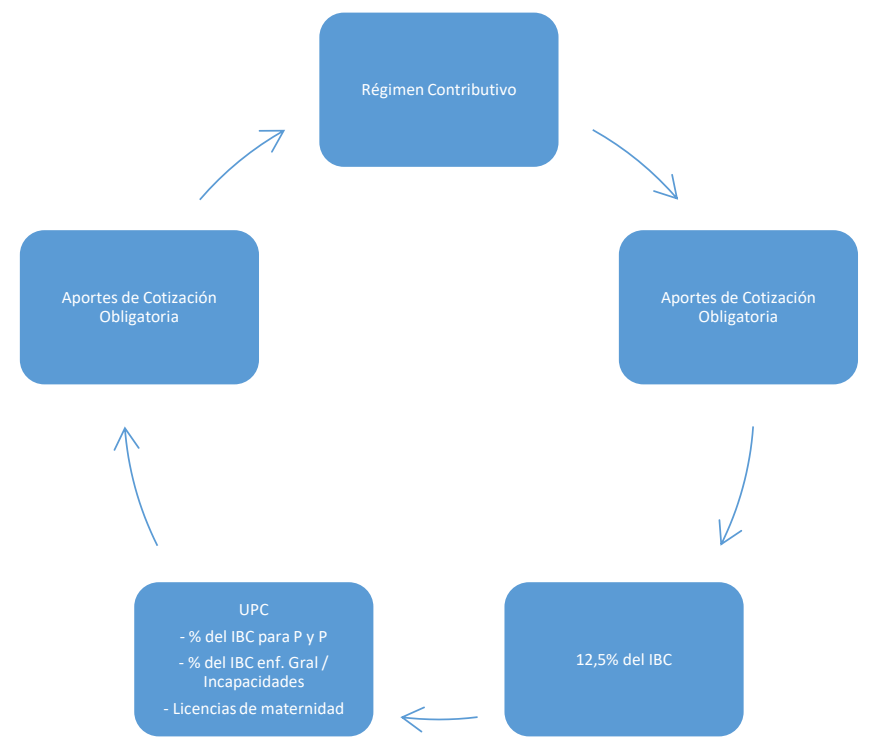

Fuente: Elaborado por el Autor con base en fuentes y usos del Ministerio de Salud

La posibilidad de comparar varias unidades de servicios hospitalarios (Decision Making Unit) como unidades donde se toman decisiones en condiciones de mercado comparables despiertan el interés por encontrar, en el ámbito de la gestión pública, la maximización de la utilización de los insumos, teniendo en cuenta la demanda de servicios.

La eficiencia de los hospitales depende de la disposición que se haga de los recursos disponibles y por supuesto del costo de estos, por lo tanto, desde el punto de vista neoinstitucional se conforma una combinación específica de capital (K) y trabajo (L) que se puede definir en una función de producción que presenta combinaciones de los insumos, por ejemplo: consultorios y médicos cuyo producto es la cantidad de consultas. 
Según Pinzón (2003), la productividad de los servicios de salud de los hospitales se ve afectada por los altos costos (crecimiento en el gasto), los cuales se asocian con la nivelación salarial, la cual no se refleja en el crecimiento de la producción, y termina afectando el desempeño de los hospitales

Asimismo, Wiesner (1998) señala que el Estado, como institución, es responsable de la competencia y los mercados, es el moderador del impulso que permite maximizar el bienestar individual y colectivo. Razón por la cual, sin la intervención de este, se sacrificaría tanto la eficiencia hospitalaria como una mayor equidad desde el punto de vista social. Por ello el estudio de las instituciones es indispensable para entender los resultados reales que surgen de los mercados.

Los beneficios se definen como los ingresos menos los costos (Varian, 1999), se pueden expresar de la siguiente forma:

$$
\prod=\sum_{i=1}^{n} p_{i} y_{i}-\underbrace{m} w_{i=1}^{m} x_{i}
$$

Ingresos Costos

Los términos productividad y eficiencia con frecuencia se utilizan como sinónimos cuando se trata de medir el desempeño de un proceso, sin embargo, es conveniente diferenciarlos. La productividad se entiende como la relación entre lo producido y los medios empleados, en sentido técnico se entiende como el incremento de la cantidad de outputs cuando varían los elementos que hacen parte de los imputs; la primera vez que se hizo referencia a este concepto fue en 1766 en la obra de Quesnay, economista francés, pionero del pensamiento económico, quien afirmó que "la regla de conducta fundamental es conseguir la mayor satisfacción con el menor gasto o fatiga"8.

La eficiencia técnica varía entre 0 y 1 , un valor de 1 indica que la empresa es completamente eficiente y opera en la frontera de producción; un valor menor que uno refleja que la empresa opera por debajo de la frontera y la diferencia entre 1 y el valor observado mide la ineficiencia técnica (Coelli, Estanche, Perelman, \& Trujillo, 2003).

\footnotetext{
${ }^{8}$ Quisney, médico de profesión, economista de la escuela fisiocrática del siglo XVIII
} 
Para medir la eficiencia de las organizaciones se han utilizado diferentes métodos, tales como: índices basados en precios mediante técnicas econométricas (frontera estocástica) ${ }^{9}$ (Canay, 2001), y análisis envolvente de datos.

Los hospitales requieren de una revisión más ajustada de las técnicas descritas, debido a que cuentan con un modelo de administración que requiere que tres áreas se conjuguen armónicamente, estas son:

Figura 3 Modelo de Administración Hospitalario

\section{Administración $=$ \\ Parte financiera Financiera

Fuente:Elaborado por el autor, 2017

La parte más visible e importante frente al objeto social es por supuesto el área científica, pero para que esta pueda operar con solvencia, requiere se le proporcionen los recursos de personal, insumos, dispositivos y equipos médicos, y áreas habilitadas y dispuestas para llevar a cabo los procedimientos habilitados de acuerdo con el nivel de atención.

Esto significa que cada uno de estos aportes es susceptible de ser optimizado, en cuyo caso los hospitales deben ajustar los modelos de optimización a los siguientes parámetros.

$$
O H=\beta_{1} \text { Financiera }+\beta_{2} \text { Científica }+\varepsilon
$$

\section{$\mathrm{OH}=$ Optimización Hospitalaria}

El marco conceptual da señales de que existe evidencia de se requieren tres factores por revisar.

\footnotetext{
${ }^{9}$ Las estimaciones de fronteras de producción son una extensión de las típicas estimaciones de funciones de producción microeconómicas, basadas en la premisa de que esta función de producción representa algún tipo de ideal, la máxima cantidad de producto asequible dados los insumos De esta forma, en la práctica las fronteras de producción no son más que una regresión que se ajusta a los datos reconociendo la restricción de que todas las observaciones deben encontrarse debajo de la frontera y al menos una debe estar sobre ella
} 


\subsection{Marco Legal}

El Artículo 49 de La Constitución Política de Colombia modificado por el Artículo 1 del Acto Legislativo 2 de 2009, establece que "la atención de la salud y el saneamiento ambiental son servicios públicos a cargo del Estado"

Sin embargo, la Ley Estatutaria 1751 de 2015 (desarrollada para regular un derecho fundamental para un derecho considerado de tercera generación, no por ser menos importante, sino porque fue considerado derecho fundamental tiempo después) considera en el Artículo 5. Obligaciones del Estado, considera en el inciso d) Establecer mecanismos para evitar la violación del derecho fundamental a la salud en todo el territorio nacional...

La salud como derecho fundamental de la Ley Estatutaria es una refrendación de la Sentencia de la Corte Constitucional T-760 de 2008 en la cual la Corte Constitucional con el fin inicial de generar jurisprudencia, sobre las recurrentes tutelas de los usuarios decidió pronunciarse sobre la salud (derecho conexo con la vida hasta antes de la sentencia) y definirlo como un derecho fundamental.

Las implicaciones económicas de los alcances de la salud como derecho fundamental no se vislumbran en los alcances de la ley estatutaria, es decir que la jurisprudencia se encargó del problema de la cobertura del servicio (como estaba considerada antes) pero no se preocupó por establecer nuevas fuentes de financiamiento por eso es que se considera una refrendación del derecho fundamental a la salud, finalmente la Corte Constitucional la declaró exequible mediante la sentencia C-634 de 2015.

Antes de la Ley 1751 de 2015 se habían hecho modificaciones importantes al Sistema General de Seguridad Social en Salud - SGSSS, por ejemplo en 2011 la Ley 1438 modificó parcialmente la Ley 100 de 1993 por medio de la cual se creó el Sistema de Seguridad Social Integral, principalmente se establecieron algunos cambios orgánicos, por ejemplo, se constituyó el Consejo Nacional de Seguridad Social, como organismo de dirección del SGSSS, se integraron en todos los niveles territoriales, las instituciones de dirección, las entidades de promoción y prestación de servicios de salud, así como el conjunto de acciones de salud y control de los factores de riesgo en su respectiva 
jurisdicción y ámbito de competencia, se establecieron fuentes de financiación de transferencias de inversión social y de impuestos a las armas y municiones.

La Ley 100 de 1993 "Por la cual se crea el sistema de seguridad social integral y se dictan otras disposiciones" es la ley que se encarga de dar forma al Sistema General de Seguridad Social en Salud como hoy se conoce, por lo menos en la parte estructural. La ley 100 define cuatro grandes elementos que son:

El Sistema general de Pensiones,

El Sistema General de Seguridad Social en Salud

El Sistema General de Riesgos Profesionales

Los servicios sociales complementarios.

La ley permite la participación del sector privado en la prestación de servicios de salud a los trabajadores, que hasta 1991 era monopolio del Seguro Social, crea el Fondo de Solidaridad y Garantía para asegurar el sector subsidiado y admite la intervención de un mercado regulado cuando le permite a los trabajadores escoger la Entidad Prestadora de Salud.

El concepto fundamental del sistema se reconoce en un conjunto de actividades, procedimientos, suministros que se brinda a los usuarios para mantenerse saludables o para recuperar la salud

La Ley 100 también establece el régimen jurídico para las empresas sociales de salud en el artículo 195, las definiciones establecen por ejemplo que en materia contractual las ESE se rigen por el derecho privado, que el régimen presupuestal será el que prevea en función de su especialidad, en la ley orgánica de presupuesto y que podrá recibir transferencias directas de los presupuestos de las entidades territoriales; en materia tributaria se someterá al régimen previsto para los establecimientos públicos; y dispone los términos generales en materia de gobernanza.

En materia de cobertura con la ley 1438 de 2011 se admitieron planes complementarios en las que el afiliado paga por los servicios de salud que requiere y en ese sentido, los usuarios que pagan acceden a servicios con mayor celeridad que quienes no están en condiciones de hacerlo, es decir que se legalizaron los gastos de bolsillo y tratamiento para diferencial para los usuarios. 
Tabla 2 Tabla de Resumen Legal

\begin{tabular}{|c|c|c|}
\hline AÑO & Ley o Norma & Alcance \\
\hline 1993 & Ley 100 de 1993 & Creación y Organización del SGSSS \\
\hline 1997 & Acuerdo 17 de 1997 & $\begin{array}{l}\text { Por el cual se transforman los } \\
\text { Establecimientos Públicos Distritales } \\
\text { Prestadores de Servicios de Salud Como } \\
\text { Empresas Sociales del Estado }\end{array}$ \\
\hline 2008 & Sentencia T-760 de 2008 & $\begin{array}{l}\text { Jurisprudencia sobre las recurrentes tutelas } \\
\text { en la cual define la salud como un derecho } \\
\text { fundamental }\end{array}$ \\
\hline 2009 & $\begin{array}{l}\text { Artículo } 49 \text { de la Constitución } \\
\text { Política, modificado por el } \\
\text { Acto legislativo } 2 \text { de } 2009\end{array}$ & La Salud un servicio público \\
\hline 2011 & Ley 1438 de 2011 & Modificación parcial de la ley 100 de 1993 \\
\hline 2011 & Decreto 971 de 2011 & $\begin{array}{l}\text { Se define el instrumento por medio del cual se } \\
\text { girarán los recursos del régimen subsidiado a } \\
\text { las EPS }\end{array}$ \\
\hline 2012 & Resolución 710 de 2012 & $\begin{array}{l}\text { Metodología para la elaboración del plan de } \\
\text { gestión por parte de los Gerentes }\end{array}$ \\
\hline 2013 & Ley 1608 de 2013 & $\begin{array}{l}\text { Por medio del cual se adoptan medidas para } \\
\text { mejorar la liquidez y el uso de algunos } \\
\text { recursos del sector salud }\end{array}$ \\
\hline 2013 & Resolución 743 de 2013 & $\begin{array}{l}\text { Modificación a la resolución } 710 \text { de } 2012 \\
\text { sobre calificación al plan de gestión }\end{array}$ \\
\hline 2015 & Ley Estatutaria 1751 & La Salud y derecho fundamental \\
\hline 2015 & Sentencia C-634 de 2015 & Exequibilidad de la Ley 1751 \\
\hline
\end{tabular}

Fuente: Elaborado por el autor con base en información de la Secretaría Jurídica Distrital de la Alcaldía Mayor de Bogotá

Como conclusiones preliminares se puede explicar que parte de la crisis de la salud se fundamenta en las asimetrías que se generan con los planes complementarios; los desarrolladores de política solucionaron sus problemas particulares, los principios de equidad y justicia que requiere el "public choice" fueron violentados, por ese motivo ante la crisis de la salud, no existe una urgente necesidad de respuesta, se violentó el "velo de ignorancia" debido a que de acuerdo con la propuesta de Rawls, "El velo de ignorancia", 
que quienes toman la decisión (en este caso la decisión pública), conocen el funcionamiento de las instituciones, conocen las leyes fundamentales de la economía y lo único que no conocen es el lugar que cada uno ocupará en la sociedad, en cuyo caso ninguno debiera delinear principios que favorezcan su condición particular (Rawls, 1971). Sin embargo, los "police maker" resolvieron en buena medida el acceso a servicios de salud, pagando por recibir atención de calidad, en la que terminan de todas maneras facturando a las EPS los servicios que tengan cobertura, situación a la que no puede acceder la mayoría.

Se vulneró el principio mínimo de equidad porque se permitió que existieran pacientes con categorías diversas y por supuesto, calidades de atención diversas. Pacientes de primera, segunda y tercera categoría.

La Constitución Política de Colombia, Título II, Artículo 13 establece que "Todas las personas nacen libres e iguales ante la ley, recibirán la misma protección y trato de las autoridades y gozarán de los mismos derechos, libertades y oportunidades sin ninguna discriminación por razones de sexo, raza, origen nacional o familiar, lengua, religión, opinión política o filosófica.", motivo por el cual es difícil explicar las categorías de los pacientes del párrafo anterior, la distancia entre lo normativo y lo positivo tiene la siguiente consonancia.

Frente al Sistema General de Seguridad Social todos somos iguales, frente a la forma de solucionar los problemas de salud es que surgen las diferencias, quienes cuentan con recursos, con gastos de bolsillo cubren las deficiencias del sistema o pagan por acceder a mejores servicios, estos serían los pacientes de primera; quienes pueden con recursos de bolsillo hacer pagos parciales, a cambio de velocidad de respuesta en sus requerimientos, principalmente para recuperar su estado saludable, son pacientes de segunda y quienes están a merced del sistema y tienen que esperar son quienes en esta lógica se consideran pacientes de tercera.

Los fallos en la regulación eficiente de los servicios públicos pueden derivar una situación peor que el punto de partida; ocurre cuando no se cuenta con un marco regulatorio adecuado y se termina promoviendo actividades poco eficientes para la mayoría, esta situación puede darse cuando se materializa el riesgo de captura del regulador, sea por 
cuenta de los grupos de presión que se pueden beneficiar con la decisión o cuando el regulador detecta beneficios (Stigler, 1961).

La crisis del sistema de salud es cada vez más pronunciada, la lenta respuesta del regulador obedece al confort sobre la posición que frente al sistema tiene el regulador y la presión que ejercen los grupos de interés por mantener el "Statu Quo"; la principal causa de la crisis fiscal radica en la contradicción de subordinar a fuerzas del mercado de un derecho fundamental como es el acceso a la salud mientras los medios de producción con mayor desarrollo se encuentran en manos de los privados con una conclusión importante, se socializan las pérdidas pero se privatizan las utilidades (O Connor, 2002) 


\title{
1.5. ANALISIS DEL ENTORNO DE LA RED HOSPITALARIA DE BOGOTA, EN TERMINOS ADMINISTRATIVOS, FINANCIEROS Y GESTIÓN HOSPITALARIA
}

\author{
"Los mercados no siempre operan de forma eficaz, \\ porque compradores y vendedores no siempre tienen \\ acceso a la información que requieren para tomar \\ decisiones óptimas”. Joseph Stiglitz
}

Ampliando la propuesta de Canay para medir la eficiencia institucional resulta conveniente explicar la situación de las Instituciones Prestadoras de Servicios de salud en el Distrito en el periodo 2006-2015 para aproximar las pretensiones del objetivo principal al objeto de estudio.

La Red Hospitalaria puede ser organizada de varias formas; por distribución geográfica, se conoce como red norte, red sur, red sur occidente y red centro oriente. La distribución garantiza que exista al menos un hospital de tercer nivel de atención (alta complejidad) y uno de segundo nivel de atención que sirven como centros de referencia para la asistencia especializada y complementaria de la población, con un total de 22 ESE distribuidos en las 20 localidades del Distrito. Adicionalmente al Red Pública también pertenece el Hemocentro Distrital, institución que actúa como apoyo y garante del suministro de sangre y hemoderivados (Salud Capital, 2017).

Tabla 3 Composición de la Red Pública Hospitalaria

\begin{tabular}{|c|c|c|c|}
\hline $\begin{array}{l}\text { Red Centro } \\
\text { Oriente }\end{array}$ & Red Norte & Red Sur & $\begin{array}{l}\text { Red Sur } \\
\text { Occidente }\end{array}$ \\
\hline $\begin{array}{l}\text { - Centro } \\
\text { Oriente } \\
\text { - La Victoria } \\
\text { - San Blas } \\
\text { - San Cristobal } \\
\text { Rafael Uribe } \\
\text { - Santa Clara }\end{array}$ & $\begin{array}{l}\text { - Usaquén } \\
\text { - Simon Bolivar } \\
\text { - Chapinero } \\
\text { - Engativa } \\
\text { - Suba }\end{array}$ & $\begin{array}{l}\text { - Tunjuelito } \\
\text { - Tunal } \\
\text { - Vista } \\
\text { Hermosa } \\
\text { - Meissen } \\
\text { - Usme } \\
\text { - Nazareth }\end{array}$ & $\begin{array}{l}\text { - Pablo VI Bosa } \\
\text { - Bosa } \\
\text { - Occidente de } \\
\text { Kennedy } \\
\text { - Fontibon } \\
\text { - Sur }\end{array}$ \\
\hline
\end{tabular}

Fuente: Elaborado por el autor con información del Fondo Financiero Distrital de Salud, 2017. 


\subsubsection{Aspectos Administrativos}

El Sistema General de Seguridad Social para el Distrito de acuerdo con la condición de aseguramiento de la población, y orientada a la universalización de la protección social en salud atiende población con diferencias en la vinculación al sistema de los regímenes contributivo, subsidiado y especiales (Fuerzas armadas, Magisterio, Ecopetrol)

Al régimen contributivo pertenecen los trabajadores dependientes (con contrato de trabajo), los independientes con capacidad de pago (se incluyen los contratistas por Orden de Prestación de Servicios) y los pensionados; cotizantes y beneficiarios afiliados a una Empresa Promotora de Salud (EPS-C).

En el régimen subsidiado se atienden las personas sin capacidad de pago, a quienes se les financia a través del Estado con afiliación a una Empresa Promotora de servicios de Salud (EPS-S)

En los regímenes de excepción se atienden los empleados de las Fuerzas Armadas, Magisterio y Ecopetrol, entre otros que cuentan con coberturas y procedimientos diferentes.

Las afiliaciones de los usuarios a estos regímenes son mutuamente excluyentes, es decir que un usuario no debería estar afiliado sino a un solo régimen.

Aún queda una parte de la población que por diferentes circunstancias (generalmente en condiciones de pobreza extrema) no se encuentra afiliada al sistema de salud, esta se atiende en la Red Pública de Hospitales del Distrito, y se factura por una cuenta de población pobre no asegurada que el ente territorial traslada como pago a las IPS, sin embargo, en los años recientes se ha promovido la vinculación al régimen subsidiado y los giros por este concepto han bajado de forma importante como se visualizará en los aspectos financieros.

Las personas pertenecientes a poblaciones especiales son certificadas por los organismos competentes y se encuentran exentas de aplicar la encuesta SISBEN para acceder al Régimen Subsidiado, así como del pago de cuotas de recuperación y copagos los cuales se reportan mediante los "listados Censales" como: 
Población infantil abandonada o desvinculada del conflicto armado, a cargo del Instituto Colombiano de Bienestar Familiar (ICBF); Población víctima del conflicto armado (Unidad Nacional para la Atención y Reparación Integral a las Víctimas); Población desmovilizada (Agencia Colombiana para la Reintegración); Personas mayores en centros de protección (Secretaría Distrital de Integración Social); Población Gitana-ROM (autoridad legítimamente constituida). - Comunidades Indígenas: Muisca de Suba, Muisca de Bosa, Inga, Ambiká-Pijao y Kichwa (autoridades tradicionales y legítimas).

Habitantes de la Calle (Secretaría Distrital de Integración Social). • Población reclusa. (Cárcel Distrital). - Personas con discapacidad en protección (Secretaría Distrital de Integración Social).

Una de las formas de organizar los Hospitales del Distrito es por Niveles de atención. Como el propósito del presente documento es evaluar la eficiencia de la Red Pública Hospitalaria, la forma más conveniente es agrupar mediante características similares, motivo por el cual se agrupan por niveles de atención de la siguiente manera.

Tabla 4 Composición de la Red Pública de Hospitales por Niveles de Atención

$\quad$ I Nivel
-Usme
-Vista Hermosa
-San Cristobal
-Chapinero
-Usaquén
-Del Sur
- Nazareth
-Pablo VI Bosa
-Uribe Uribe
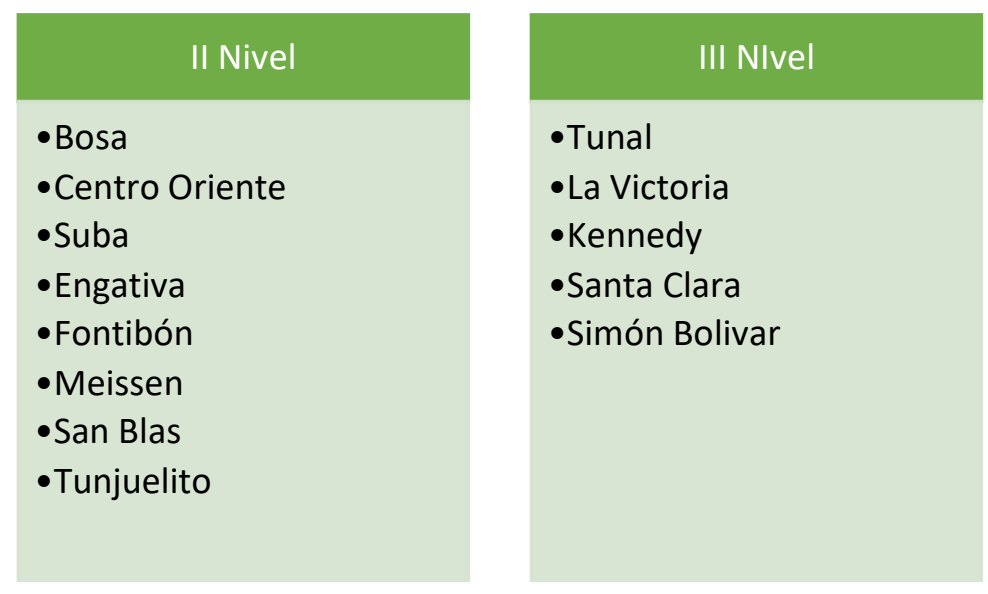

Fuente: Elaborado por el autor con base en el Fondo Financiero Distrital de Salud 2015

Los hospitales públicos fueron transformados en ESE y en consecuencia gozan de autonomía administrativa, patrimonio propio, personería jurídica, y de independencia financiera, esto significa que deben atender leyes de mercado en la generación de sus ingresos y adoptar el peso de la representación del Estado frente a la demanda insatisfecha de servicios de salud de los usuarios, en una dinámica en la que el que recibe el servicio no paga, el que paga no ordena y el que ordena elude prestar el servicio con el fin de que 
su cartera no se desborde. En esa medida los incentivos naturales de mercado son inexistentes; las asimetrías de información, riesgo moral y selección adversa no permiten configurar papeles bien definidos de oferta y demanda.

Las Empresas Sociales del Estado se convierten en agentes que no logran reflejar las características que se les exige en su nombre corporativo (Verbi gracia "Hospital Meissen II Nivel ESE), es decir, no son propiamente una empresa porque el objetivo básico financiero está subordinado a la prestación de un servicio público ahora considerado derecho fundamental, tal como se reza la Sentencia T121 de 2015 de La Corte Constitucional (Sentencia T-121/15, 2015).

Adicionalmente la administración pública se diferencia de la administración privada en cuanto al fin perseguido y a los medios de acción que utiliza. Mientras la administración privada se propone un fin egoísta, representado en un interés particular, la administración pública persigue un interés general, representado en el bien común (Rodríguez R., 2008).

Las ESE no se pueden considerar Sociales por la disyuntiva que existe en el concepto al que se acude en la creación de las mismas; se consideran sociales por cuanto por medio de ellas se prestan servicios de salud y constituyen una "categoría especial de entidad pública descentralizada”, con personería jurídica, patrimonio propio y autonomía administrativa (Congreso de la República de Colombia, 1993), las características del régimen especial según el Artículo 15 del Decreto 1876 de 1994 establece que estas empresas estarán sujetas, por regla general, al régimen jurídico propio de las personas de derecho público, mientras que el artículo 16 ibid. estipula que en materia de contratación se aplicarán las normas del derecho privado y los contratos estarán sujetos a la contratación ordinaria, el propósito (interpretación del autor) es facilitar los mecanismos de contratación para adquirir materiales e insumos médicos de los cuales pueda depender una vida, y sin embargo, transfiere la obligación del Estado a manos de la administración de turno, la obligación de velar por la vida de los usuarios con una clara asimetría entre transferencia de obligaciones y flujo de recursos.

Las ESE tampoco se pueden considerar del Estado en el estricto sentido, ya que la Ley 1438 de 2011, como dice Franco, "le podría a uno hacer pensar, a la ligera, que la ley 1438 nos permite, a diferencia del pasado, mostrar una transición de un sistema de salud 
basado en el mercado hacia otro más estatista" (Franco Giraldo, 2011); adicionalmente en las disposiciones generales de la Ley se establece que los lineamientos relacionados con las finanzas son en un marco de sostenibilidad, por lo tanto, en buena medida controvierten el espíritu de lo social y la posibilidad de que el Estado haga presencia con transferencia de recursos (aunque los canales legales de presupuesto lo permitan), mediante subsidios a la oferta con el fin de garantizar la prestación de servicios. Estas consideraciones hacen parte del Statu Quo de las Empresas Sociales del Estado, que se obligan en el marco de autosostenibilidad que se les exige, a generar sus propios ingresos; con los esfuerzos que supone lograr recursos suficientes para atender la demanda espontánea de servicios de salud en donde existe una regulación que repele las fuerzas del mercado.

Con la entrada en vigencia de la Ley 1438 de 2011, "Por medio de la cual se reforma el Sistema General de Seguridad Social en Salud y se dictan otras disposiciones", amparado en el artículo 80, el Ministerio de Salud y Protección Social inició la evaluación y divulgación de riesgo de las Empresas Sociales del Estado, y adicionalmente, se dispuso que las que fueren categorizadas con riesgo medio o alto deberían someterse a un Programa de Saneamiento Fiscal y Financiero ante el Ministerio de Hacienda y Crédito Público.

El Ministerio de Salud ha expedido las siguientes resoluciones:

Tabla 5 Resumen de Resoluciones de calificación de riesgo de las ESE Ministerio de Salud

\begin{tabular}{|l|l|}
\hline Resolución 2509 de 2012 & Evalúa resultados de 2011 \\
\hline Resolución 1877 de 2013 & Evalúa resultados de 2012 \\
\hline Resolución 2090 de 2014 & Evalúa resultados de 2013 \\
\hline Resolución 1893 de 2015 & Evalúa Resultados de 2014 \\
\hline Resolución 2184 de 2016 & Evalúa Resultados de 2015 \\
\hline
\end{tabular}

Fuente: Ministerio de Salud y Protección Social

Los resultados de 2016 se evaluaron con la resolución 1755 de 2017, en los considerandos cuenta con un párrafo en el que se explica que la Red Pública Hospitalaria de Bogotá ha entrado en un proceso de reorganización en el que se han fusionado los 22 Hospitales en 
4 Empresas Sociales del Estado, motivo por el cual, el 26 de mayo de 2017 no se publicaron resultados del Distrito Capital.

\subsubsection{Aspectos Financieros}

Se considera que el principal problema de los hospitales desde la perspectiva financiera es el crecimiento y deterioro de la cartera; en el presente trabajo se presentan en pesos constantes de 2015, es decir eliminada la distorsión de la inflación en las cifras, se puede apreciar un incremento de la cartera en la mayoría de los hospitales, visualizado a partir de las cifras agregadas y como consecuencia de la salida del mercado de importantes EPS que no han pagado por los servicios recibidos por sus abonados, y que los términos legales de una liquidación señalan un orden para atender con los recursos disponibles a los acreedores, en los que las IPS van en el tercer orden de importancia, primero van los impuestos, luego los trabajadores y por último los acreedores (Congreso de la República de Colombia, 2006).

Del mercado han salido las siguientes EPS

Tabla 6 EPS liquidadas

\begin{tabular}{|c|c|c|c|c|}
\hline CODIGO & NIT & ENTIDAD PROMOTORA DE SALUD EPS & & VIGENCIA \\
\hline EPS020 & $\begin{array}{l}899999026- \\
0\end{array}$ & $\begin{array}{l}\text { Caja de Previsión Social de Comunicaciones } \\
\text { Caprecom Entidad Promotora de Salud }\end{array}$ & Liquidada & \\
\hline EPS013 & $\begin{array}{l}800250119- \\
1\end{array}$ & Saludcoop E.P.S & Liquidada & $\begin{array}{l}\text { Fecha de liquidación: } \\
\text { 01/12/2015 } \\
\text { Para pagos de liquidaciones con } \\
\text { periodo } 2015-11 \text { o anteriores en } \\
\text { salud se puede seguir realizando } \\
\text { el aporte a SaludCoop. }\end{array}$ \\
\hline EPS024 & $\begin{array}{l}830130800- \\
4\end{array}$ & Cajanal E.P.S. & Liquidada & \\
\hline EPS006 & $\begin{array}{l}860013816- \\
1\end{array}$ & Instituto de Seguros Sociales & Liquidada & \\
\hline EPS035 & $\begin{array}{l}830096513- \\
1\end{array}$ & Redsalud Atención Humana EPS S.A. & Liquidada & $\begin{array}{l}\text { Fecha de liquidación: } \\
01 / 07 / 2011 \\
\text { Para pagos de liquidaciones con } \\
\text { periodo anterior en salud hacia } \\
\text { atrás dirigir el aporte al Fosyga } \\
\text { MIN001. }\end{array}$ \\
\hline EPS034 & $\begin{array}{l}805021984- \\
2\end{array}$ & Saludcolombia EPS S.A & Liquidada & \\
\hline EPS015 & $\begin{array}{l}860512237- \\
6\end{array}$ & Salud Colpatria S.A. EPS & Liquidada & $\begin{array}{l}\text { Fecha de liquidación: } \\
01 / 08 / 2013 \\
\text { Para pagos de liquidaciones con } \\
\text { periodo anterior en salud hacia } \\
\text { atrás dirigir el aporte al Fosyga } \\
\text { MIN001. }\end{array}$ \\
\hline
\end{tabular}




\begin{tabular}{|c|c|c|c|c|}
\hline EPS038 & $\begin{array}{l}900112778- \\
7\end{array}$ & Multimédicas Salud con Calidad EPS S.A. & Liquidada & \\
\hline EPS014 & $\begin{array}{l}830006404- \\
0\end{array}$ & Humana Vivir EPS & Liquidada & $\begin{array}{l}\text { Fecha de liquidación: } \\
01 / 09 / 2013 \\
\text { Para pagos de liquidaciones con } \\
\text { periodo anterior en salud hacia } \\
\text { atrás dirigir el aporte al Fosyga } \\
\text { MIN001. }\end{array}$ \\
\hline EPS009 & $\begin{array}{l}890900842- \\
6\end{array}$ & Comfenalco Antioquia EPS & Liquidada & \\
\hline EPS026 & $\begin{array}{l}804001273- \\
5\end{array}$ & Solidaria de Salud Solsalud S.A & Liquidada & \\
\hline EPS039 & $\begin{array}{l}900074992- \\
3\end{array}$ & Golden Group EPS & Liquidada & \\
\hline EPSC20 & 899999026 & $\begin{array}{l}\text { Caja de Previsión Social de Comunicaciones } \\
\text { EPS Caprecom }\end{array}$ & Liquidada & \\
\hline CCFC10 & 860007336 & $\begin{array}{l}\text { Caja Colombiana de Subsidio Familiar } \\
\text { Colsubsidio EPSS }\end{array}$ & Liquidada & $\begin{array}{l}\text { Fecha de liquidación: } \\
01 / 11 / 2015 \\
\text { Para pagos de liquidaciones con } \\
\text { periodo anterior en salud hacia } \\
\text { atrás dirigir el aporte al Fosyga } \\
\text { MIN001. }\end{array}$ \\
\hline CCFC18 & 860013570 & Caja de Compensación Familiar Cafam EPS & Liquidada & $\begin{array}{l}\text { Fecha de liquidación: } \\
01 / 11 / 2015 \\
\text { Para pagos de liquidaciones con } \\
\text { periodo anterior en salud hacia } \\
\text { atrás dirigir el aporte al Fosyga } \\
\text { MIN001. }\end{array}$ \\
\hline
\end{tabular}

Fuente: Elaborada por el autor con base en información de aportes en línea del Ministerio de Salud y

Protección Social.

Las EPS que no cuentan con resolución de liquidación es porque aún se encuentran en proceso de liquidación, la resolución de liquidación se considera el último acto jurídico que desarrollan las empresas.

Existe una metodología de evaluación de riesgo para las ESE que fue definida en la Resolución 2509 de 2012, que fue la primera, luego de que la Ley 1438 de 2011 entrara en vigor y se define como información para la estimación de riesgo el siguiente procedimiento:

Primero, el déficit o superávit corriente:

$$
D S O C=I O C R-G O C C
$$

Segundo, el déficit o superávit no corriente:

$$
D S O N C=E R C-P A V A
$$

Tercero, el Superávit o déficit total:

$$
D S O T=S D O C+S D O N C
$$

Con esta información se estima el índice de riesgo

$$
I R=D S O T \div \text { Ingresos operacionales totales recaudados }
$$


Tabla 7 Convenciones para estimación de riesgo

\begin{tabular}{|l|l|}
\hline DSOC & Déficit o Superávit Operacional Corriente \\
\hline IOCR & Ingresos Operacionales Corrientes Recaudados \\
\hline IOTR & Ingresos Operacionales Totales Recaudados \\
\hline GOCC & Gastos Operacionales Corrientes Comprometidos \\
\hline DSONC & Déficit o superávit operacional no corriente \\
\hline ERC & Estimación de recaudo de cartera \\
\hline PAVA & Pasivos adquiridos en vigencias anteriores \\
\hline DSOT & Déficit o superávit operacional total \\
\hline IR & Índice de Riesgo \\
\hline
\end{tabular}

Fuente: Elaborado por el Autor

Y la categorización se desarrolla con base en la siguiente tabla

Tabla 8 Parámetros definición de riesgo ESE

\begin{tabular}{|c|c|}
\hline Índice de riesgo & Categorización de riesgo \\
\hline a. Mayor o igual que 0,0 & Sin riesgo \\
\hline b. Entre menos 0,01 y 0,10 & Riesgo bajo \\
\hline c. Entre menos 0,11 y menos 0,2 & RM \\
\hline $\begin{array}{l}\text { d. Ingresos reconocidos menos } \\
\text { gastos comprometidos menor que } \\
\text { cero }(0)\end{array}$ & RA \\
\hline
\end{tabular}

Fuente: Resolución 710 de 2012 del Ministerio de Salud y Protección

La metodología de medición de riesgo se considera acertada desde la perspectiva financiera debido a que tal como se va a presentar en lo sucesivo del presente capítulo, las Empresas Sociales del Estado tienen la obligación de adelantar la información financiera como cualquier empresa, es decir en cinco estados financieros, que son el balance, el estado de resultados, estado cambios en la situación del patrimonio, el estado de cambios en la situación financiera y el estado de flujos de efectivo los cuales deben ir acompañados de las notas a los estados financieros o revelaciones tal como lo exige el artículo 22 del Decreto 2469 de 1993 que establece el reglamento general de contabilidad, sin embargo, la medición de riesgo se remite a aspectos que buscan claramente establecer 
el nivel de déficit o superávit que se remite a la caja, es decir, a temas de presupuesto el cual es definido por el Decreto 111 de 1996.

Con base en las Resoluciones expedidas se ha extraído y adelantado una clasificación de riesgo por código de colores (emulando código NFPA) ${ }^{10}$, resultado de las evaluaciones de riesgo, para la red hospitalaria del Distrito Capital por niveles de atención:

Tabla 9 Evolución del Riesgo Hospitales por Niveles

\begin{tabular}{|l|c|c|c|c|c|}
\hline \multicolumn{1}{|c|}{ PRIMER NIVEL } & $\begin{array}{c}2012 \\
\text { Resolución- } \\
2509\end{array}$ & $\begin{array}{c}2013 \\
\text { Resolución- } \\
1877\end{array}$ & $\begin{array}{c}2014 \\
\text { Resolución- } \\
2090\end{array}$ & $\begin{array}{c}2015 \\
\text { Resolución- } \\
1893\end{array}$ & $\begin{array}{c}2016 \\
\text { Resolución- } \\
2184\end{array}$ \\
\hline Hospital Usme & RA & RA & RA & SR & RB \\
\hline Hospital del Sur & RA & RA & RA & SR & RB \\
\hline Hospital Usaquén & RM & RM & RM & SR & RB \\
\hline Hospital Chapinero & RM & RM & RM & RM & SR \\
\hline $\begin{array}{l}\text { Hospital Rafael Uribe } \\
\text { Uribe }\end{array}$ & RM & RM & RM & SR & RB \\
\hline Hospital San Cristobal & RB & SR & SR & SR & RB \\
\hline Hospital Vista Hermosa & RB & RB & SR & SR & SR \\
\hline Hospital Nazareth & RB & RB & SR & SR & RB \\
\hline Hospital Pablo VI Bosa & RB & RB & SR & SR & SR \\
\hline
\end{tabular}

\begin{tabular}{|l|c|c|c|c|c|}
\hline \multicolumn{1}{|c|}{ SEGUNDO NIVEL } & $\begin{array}{c}2012 \\
\text { Resolución- } \\
2509\end{array}$ & $\begin{array}{c}2013 \\
\text { Resolución- } \\
1877\end{array}$ & $\begin{array}{c}2014 \\
\text { Resolución- } \\
2090\end{array}$ & $\begin{array}{c}2015 \\
\text { Resolución- } \\
1893\end{array}$ & $\begin{array}{c}2016 \\
\text { Resolución- } \\
2184\end{array}$ \\
\hline Hospital Centro Oriente & RA & RA & RA & SR & SR \\
\hline Hospital Engativá & RA & RA & RA & SR & SR \\
\hline Hospital Meissen & RA & RA & RA & SR & SR \\
\hline Hospital San Blas & RA & RA & RA & RB & RB \\
\hline Hospital Bosa & RM & RM & RM & RA & RA \\
\hline Hospital de Suba & RM & RM & RM & SR & SR \\
\hline Hospital Fontibón & RM & RM & RM & SR & SR \\
\hline Hospital Tunjuelito & RB & RB & SR & SR & SR \\
\hline
\end{tabular}

\begin{tabular}{|l|c|c|c|c|c|}
\hline \multicolumn{1}{|c|}{ TERCER NIVEL } & $\begin{array}{c}2012 \\
\text { Resolución- } \\
2509\end{array}$ & $\begin{array}{c}2013 \\
\text { Resolución- } \\
1877\end{array}$ & $\begin{array}{c}2014 \\
\text { Resolución- } \\
2090\end{array}$ & $\begin{array}{c}2015 \\
\text { Resolución- } \\
1893\end{array}$ & $\begin{array}{c}2016 \\
\text { Resolución- } \\
2184\end{array}$ \\
\hline Hospital Simón Bolívar & RA & RA & RA & SR & SR \\
\hline Hospital La Victoria & RM & RM & RM & SR & SR \\
\hline Hospital Kennedy & RB & RB & SR & SR & SR \\
\hline Hospital Santa Clara & RB & SR & SR & SR & SR \\
\hline Hospital Tunal & SR & SR & SR & SR & SR \\
\hline
\end{tabular}

${ }^{10}$ NFPA 704 es la norma que explica el "diamante de materiales peligrosos" establecido por la Asociación Nacional de Protección contra el Fuego (inglés: National Fire. Protection Association), utilizado para comunicar los riesgos de los materiales peligrosos. 
Fuente: Elaborado por el autor con base en las Resoluciones del Ministerio de Salud y Protección Social 2509 de 2012, 1877 de 2013, 2090 de 2014, 1893 de 2015 y 2186 de 2016

Las convenciones para la lectura de los niveles de riesgo es la siguiente:

Tabla 10 Convenciones de riesgo

Fuente: Elaborado por el autor

\begin{tabular}{|l|l|}
\hline RIESGO ALTO & RA \\
\hline RIESGO MEDIO & RM \\
\hline RIESGO BAJO & RB \\
\hline SIN RIESGO & SR \\
\hline
\end{tabular}

Con los colores se puede apreciar más fácilmente que en 2012, 2013 y 2014, la situación de las ESE comprometía la continuidad de algunos hospitales y amenazaba la estabilidad de la red pública hospitalaria.

En general antes de la Ley 1438, o de manera coincidente, se aprecia que existe un marcado deterioro del comportamiento financiero de los hospitales, principalmente para el II Nivel de atención ya que la mitad de los hospitales se calificaron con RA, tres mas con riesgo medio y tan solo uno, el Hospital Tunjuelito fue calificado sin riesgo. Esta situación del Hospital Tunjuelito se explica parcialmente porque esta ESE atendió el Plan de Intervenciones Colectivas que coadyuvo a mitigar el impacto del comportamiento de sus gastos con recursos frescos, generalmente pagados por anticipado. Esta misma explicación se extiende a los hospitales de I Nivel de atención, motivo por el cual solo dos hospitales fueron categorizados en riesgo alto.

La situación en la evaluación de riesgo en concordancia con el artículo 81 de la Ley 1438 de 2011, obliga a las ESE categorizadas en riesgo alto o medio a someterse y presentar un programa de saneamiento fiscal y financiero tutelado por el ente territorial, en los siguientes 60 días calendario a la expedición de la resolución.

La Secretaría de Salud tuvo que definir roles de cada ESE en la red pública con el fin de consolidar el diseño de la red distrital de servicios y que los hospitales pudieran proyectar servicios e ingresos.

El programa de saneamiento fiscal y financiero requirió la adopción de una serie de medidas como son, fortalecimiento de los ingresos, racionalización del gasto, 
reorganización administrativa, saneamiento de pasivos y reestructuración de la deuda. La intencionalidad de los programas es buena, pero en general en términos de gobernanza el fin principal era logar un respiro para la operación con la inyección de recursos de las cuentas maestras ya que las medidas descritas no constituyeron cambios realmente importantes, aunque si fueron fundamentales las definiciones de roles para consolidar lo que hoy se conoce de las redes.

La razón por la cual la situación de riesgo en general cambia para las ESE es porque los programas fueron viabilizados el 14 de marzo de 2014 por la Dirección General de Apoyo Fiscal del Ministerio de Hacienda y Crédito Público, programas que fueron acompañados por una solicitud de financiamiento de cuentas maestras por valor de $\$ 151.167$ millones de pesos (a precios corrientes de 2013), de estos recursos $\$ 72.734$ millones fueros utilizados para cubrir los pasivos de las ESE relacionados con proveedores de bienes y servicios, acreedores, litigios y demandas (Secretaría Distrital de Salud, 2015).

Existe una aproximación a la calificación de riesgo que presenta el Ministerio de Salud y es uno de los indicadores de la Resolución 710 de 2012 "Por medio de la cual se adoptan las condiciones y metodología para presentación del plan de gestión por parte de los gerentes o directores de las Empresas Sociales del Estado del orden territorial, su evaluación por parte de la Junta Directiva y se dictan otras disposiciones cuenta con un promedio de 20 indicadores; el indicador 9 es el resultado del equilibrio operacional con recaudo que se asimila bastante a la metodología del índice de riesgo. Ingresos Operacionales totales $\div$ gastos totales comprometidos

Este indicador mide la relación entre los ingresos corrientes mas cuentas por cobrar versus los gastos mas las cuentas por pagar.

Esto significa que los hospitales que no alcancen un cociente mayo o igual a 1 estarán en dificultades para cubrir los gastos comprometidos.

La evolución del indicador por niveles de atención es la siguiente:

Tabla 11 Evolución indicador 9 de la Resolución 710 de 2012 para I Nivel de Atención

\begin{tabular}{|l|r|r|r|r|r|r|r|r|r|r|r|r|r|r|}
\hline \multicolumn{1}{|c|}{ HOSPITAL } & 2002 & 2003 & 2004 & 2005 & 2006 & 2007 & 2008 & 2009 & 2010 & 2011 & 2012 & 2013 & 2014 & 2015 \\
\hline CHAPINERO & 1,01 & 1,08 & 1,1 & 0,98 & 1,49 & 1,21 & 1,25 & 1,21 & 1,35 & 1,16 & 1,12 & 1,08 & 1 & 1,04 \\
\hline USAQUEN & 0,99 & 1,01 & 1,12 & 1,06 & 0,94 & 1,04 & 1,03 & 1,02 & 1,01 & 1,01 & 0,95 & 1,06 & 1,03 & 1,04 \\
\hline HOSPITAL DEL SUR & 0,91 & 1,02 & 1,04 & 1,03 & 0,98 & 0,96 & 0,96 & 0,82 & 0,89 & 0,84 & 1,07 & 1,11 & 1,36 & 1,15 \\
\hline USME & 1,16 & 1,25 & 1,31 & 1,36 & 1,13 & 1,34 & 1,31 & 1,31 & 1,18 & 1,22 & 1,55 & 1,47 & 1,56 & 1,48 \\
\hline
\end{tabular}




\begin{tabular}{|c|c|c|c|c|c|c|c|c|c|c|c|c|c|c|}
\hline RAFAEL URIBE URIBE & 1,1 & 1,07 & 1 & 1,05 & 0,99 & 0,94 & 0,94 & 0,94 & 1 & 0,92 & 0,89 & 1,03 & 1,2 & 1,02 \\
\hline VISTA HERMOSA & 1,11 & 1,14 & 1,21 & 1,15 & 1,03 & 1,1 & 1,16 & 1,09 & 1,06 & 1,04 & 1,01 & 1,09 & 1,16 & 1,14 \\
\hline PABLO VI BOSA & 1,09 & 1,08 & 1,11 & 1,04 & 1,1 & 1,07 & 1,11 & 1,11 & 1,16 & 1,09 & 1,03 & 1,09 & 1,11 & 1,14 \\
\hline SAN CRISTOBAL & 1,05 & 1,09 & 1,2 & 1,29 & 1,19 & 1,11 & 1,11 & 1,15 & 1,3 & 1,3 & 1,28 & 1,39 & 1,52 & 1,39 \\
\hline NAZARETH & 1,05 & 1,07 & 0,97 & 1,19 & 1,03 & 1,01 & 0,89 & 0,97 & 0,95 & 1,09 & 1,32 & 1,16 & 1,14 & 1,07 \\
\hline
\end{tabular}

Fuente: Elaborado por el autor con base en la Ficha técnica SIHO

La tabla anterior explica parcialmente por qué algunos hospitales terminaron con calificación de RA, como el Hospital del Sur, Hospital Rafael Uribe Uribe, Hospital de Usaquén y Hospital de Chapinero

Tabla 12 Evolución indicador 9 de la Resolución 710 de 2012 para II Nivel de Atención

\begin{tabular}{|l|r|r|r|r|r|r|r|r|r|r|r|r|r|r|r|r|r|r|}
\hline \multicolumn{1}{|c|}{ HOSPITAL } & \multicolumn{1}{|c|}{2002} & 2003 & 2004 & 2005 & 2006 & 2007 & 2008 & 2009 & 2010 & 2011 & 2012 & 2013 & 2014 & 2015 \\
\hline Hospital Bosa & 1,03 & 1 & 0,96 & 0,94 & 0,92 & 0,93 & 0,96 & 0,91 & 0,96 & 0,88 & 2,06 & 1,75 & 1,69 & 1,72 \\
\hline Hospital Centro Oriente & 0,94 & 0,96 & 0,92 & 0,94 & 1,28 & 1,22 & 1,03 & 1,03 & 1,05 & 0,92 & 0,9 & 0,93 & 0,9 & 0,92 \\
\hline Hospital de Suba & 0,96 & 1,04 & 1,05 & 1,07 & 0,98 & 0,9 & 0,94 & 0,78 & 0,83 & 0,77 & 0,71 & 0,8 & 0,94 & 0,86 \\
\hline Hospital Engativa & 0,97 & 0,92 & 0,95 & 0,86 & 0,93 & 0,88 & 0,83 & 0,81 & 0,86 & 0,76 & 0,71 & 0,86 & 1,01 & 0,97 \\
\hline Hospital Fontibon & 0,94 & 0,98 & 1,01 & 1,15 & 0,98 & 0,96 & 0,85 & 0,71 & 0,81 & 0,86 & 0,92 & 0,93 & 1 & 0,96 \\
\hline Hospital Meissen & 0,82 & 0,93 & 0,88 & 0,91 & 0,91 & 1,08 & 0,82 & 0,76 & 0,8 & 0,68 & 0,64 & 0,74 & 1 & 0,85 \\
\hline Hospital San Blas & 0,94 & 0,97 & 1,01 & 0,98 & 0,95 & 0,89 & 0,91 & 0,9 & 1 & 0,81 & 0,79 & 0,85 & 0,87 & 0,85 \\
\hline Hospital Tunjuelito & 0,87 & 0,89 & 0,86 & 0,93 & 1,01 & 0,91 & 0,93 & 0,97 & 1 & 0,95 & 0,85 & 0,93 & 0,96 & 0,96 \\
\hline
\end{tabular}

Fuente: Elaborado por el autor con base en la Ficha técnica SIHO

La situación del II nivel es más crítica, tan solo el Hospital de Tunjuelito fue calificado sin riesgo, aunque el Hospital de Bosa es el que en la tabla dispara el comportamiento superlativo del indicador. En los segundos niveles la crisis del indicador de ingresos vs compromisos es mayor debido a que la facturación depende exclusivamente de los servicios atendidos por demanda. Adicionalmente el comportamiento de las unidades hospitalarias frente a las EPS fue desarticulada frente a la estrategia de precios. Los gerentes negociaron contratos para la prestación de servicios en una competencia poco sana para las IPS a tasas de descuento diferenciales sobre el tarifario SOAT, hasta cuando la administración distrital tomó cartas en el asunto y mediante intervención de las Juntas Directivas se establecieron topes de descuento para contratar con las ESE.

Tabla 13 Evolución indicador 9 de la Resolución 710 de 2012 para III Nivel de Atención

\begin{tabular}{|l|r|r|r|r|r|r|r|r|r|r|r|r|r|r|r|}
\hline \multicolumn{1}{|c|}{ HOSPITAL } & \multicolumn{1}{|c|}{2002} & 2003 & 2004 & 2005 & 2006 & 2007 & 2008 & 2009 & 2010 & 2011 & 2012 & 2013 & 2014 & 2015 \\
\hline Hospital Tunal & 0,85 & 0,93 & 0,91 & 0,97 & 0,89 & 0,92 & 0,97 & 0,82 & 0,74 & 0,77 & 0,72 & 0,81 & 0,85 & 0,79 \\
\hline Hospital La Victoria & 0,92 & 0,91 & 0,89 & 0,92 & 0,87 & 0,83 & 0,82 & 0,84 & 0,86 & 0,8 & 0,81 & 0,95 & 1,1 & 1,16 \\
\hline Hospital Kennedy & 0,88 & 0,89 & 0,93 & 0,91 & 0,93 & 0,89 & 0,83 & 0,79 & 0,77 & 0,77 & 0,72 & 0,86 & 0,95 & 0,83 \\
\hline Hospital Santa Clara & 0,83 & 0,97 & 0,99 & 0,93 & 0,85 & 0,88 & 0,79 & 0,85 & 0,8 & 0,73 & 0,74 & 0,91 & 1,05 & 1,02 \\
\hline Hospital Simón Bolivar & 0,89 & 0,96 & 0,95 & 0,94 & 0,89 & 0,9 & 0,9 & 0,92 & 0,97 & 0,85 & 0,76 & 0,87 & 0,97 & 0,83 \\
\hline
\end{tabular}

Fuente: Elaborado por el autor con base en la Ficha técnica SIHO 
Las ESE que en 2011 tuvieron el indicador por debajo de uno, se perfilaron para ser declarados en "RIESGO" por parte del Ministerio de Salud, en cumplimiento de la Ley 1438 de 2011; debido a que finalmente la Resolución 710 mide el déficit fiscal total (corriente + no corriente).

El ser declarado en RM o RA tuvo como consecuencia la obligación de adoptar un Programa de Saneamiento Fiscal y Financiero, que de acuerdo con la Ley 1438 de 2011, lo cual, implicaba optimizar los recursos disponibles y acceder a recursos para pagar acreencias declaradas en los estados financieros de la vigencia en la que se nominaron en riesgo. Sin embargo, nadie conocía exactamente como desarrollar un ejercicio de optimización, empezando por el calificador de riesgo.

Las situaciones particulares de gobierno corporativo de las instituciones son importantes, pero no se han tenido en cuenta porque requieren una evaluación cualitativa que no corresponde con el objeto de estudio del presente documento, motivo por el cual se pasa a la descripción de aspectos financieros que revelan las prioridades de las administraciones de turno.

Los aspectos más relevantes de la situación de riesgo de los hospitales se encuentran en la relación de incremento sistemático y deterioro de la cartera, y un consecuente incremento de las cuentas por pagar, aunque las ESE que fueron calificadas con RM o alto en 2012, tuvieron que adoptar un Programa de Saneamiento Fiscal y Financiero que con recursos de "cuentas maestras" aliviaron el peso de las cuentas por pagar que se acumularon como se aprecia en las siguientes figuras y tablas

El proceso de reconocimiento de los ingresos consiste fundamentalmente en los siguientes pasos.

Figura 4 Proceso de reconocimiento de los ingresos de las ESE

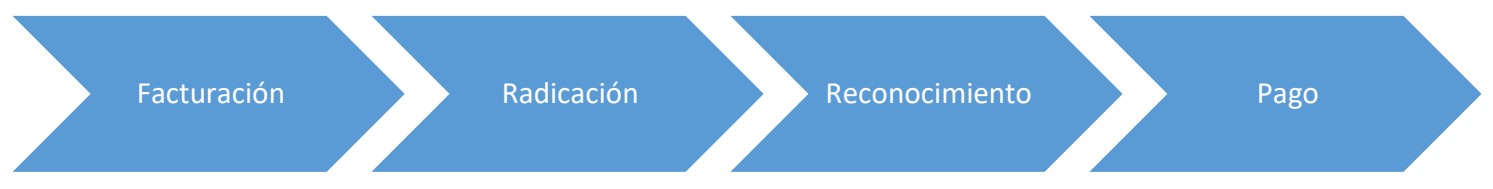

Fuente: Elaborado por el Autor 
En este procedimiento pueden ocurrir varias cosas en detrimento de la facturación. Problemas técnico-científicos o problemas administrativos.

En los problemas técnico-científicos se puede olvidar incluir en la historia médica procedimientos menores que pudieran ser cobrados. Se puede no hacer un reporte sin el cual la EPS no reconozca un procedimiento o se puede desarrollar un procedimiento sin contar con la debida habilitación (lo cual por supuesto puede ser también reconocido como un error administrativo). Desde el punto de vista administrativo, la demora en la radicación sin los soportes exigidos deriva en una de las figuras más controversiales del sistema, la glosa. La glosa de acuerdo con el proceso administrativo de la figura anterior se presenta en las dificultades del reconocimiento por parte de las EPS; consiste en la objeción sobre los servicios prestados y que se ha convertido en un tamizaje importante sobre los recursos esperados de las IPS, que sin excepción constituyen cuentas por cobrar sobre los servicios prestados.

La demora en la respuesta de glosas por parte de las IPS también se constituye en un problema administrativo para lograr el reconocimiento de los ingresos, estas se dan por varios motivos, incorrecta identificación del usuario, afiliaciones dobles, insuficiente descripción de los procedimientos, adecuada justificación de los procedimientos etc.

\subsubsection{Nivel}

El ente territorial inspirado en la mitigación de riesgo de salud de la población y respondiendo a estrategias de la Nación implementó la contratación con los Hospitales de I nivel, durante la administración del Doctor Gustavo Petro, la administración del Plan de Intervenciones Colectivas fue administrado por Hospitales de primer nivel de atención, lo que permitió que a partir de los desembolsos del ente territorial se apalancara la normal operación.

\footnotetext{
1.5.3.1. Pasivos I Nivel

Figura 5 Pasivos Totales acumulados I Nivel
} 


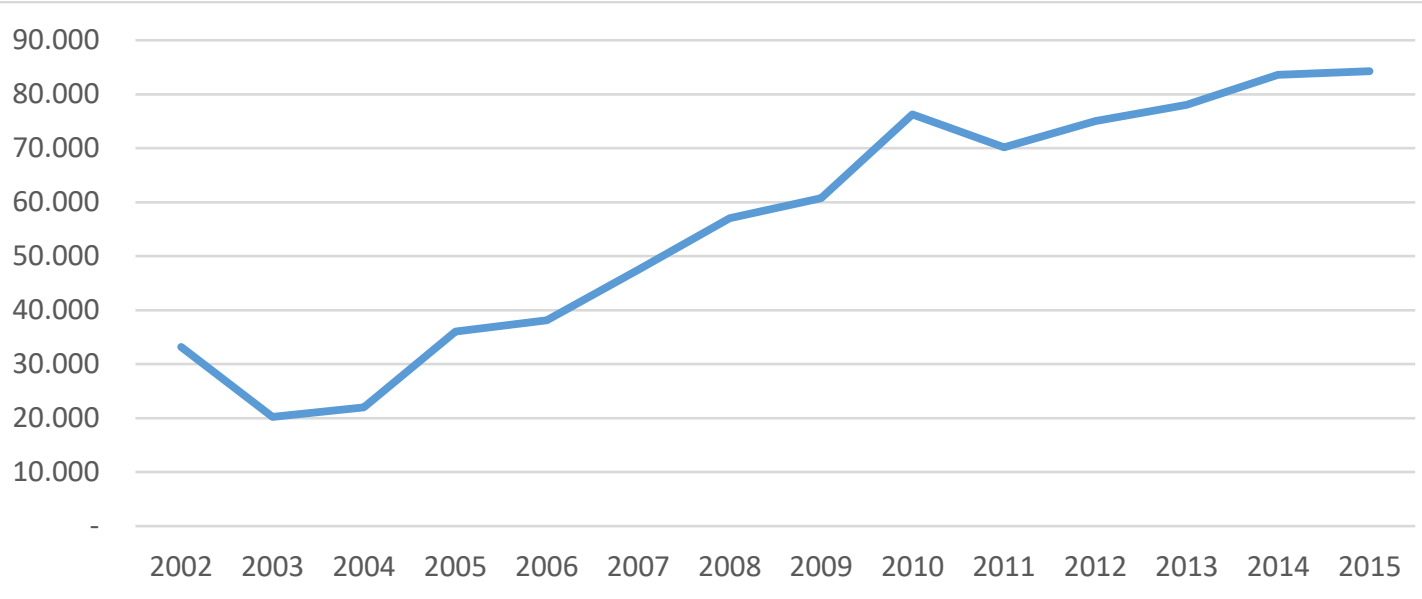

Fuente: Elaborado por el autor con base en información de las fichas técnicas del sistema de Información Hospitalario del Ministerio de Salud y Protección Social a precios constantes de 2015

El crecimiento de los pasivos es notable y en parte se explica por el incremento de la operación, tal como se muestra en la siguiente figura que refleja el comportamiento de los ingresos por venta de servicios.

\subsubsection{Ingresos I Nivel}

Figura 6 Ingresos por venta de servicios I Nivel

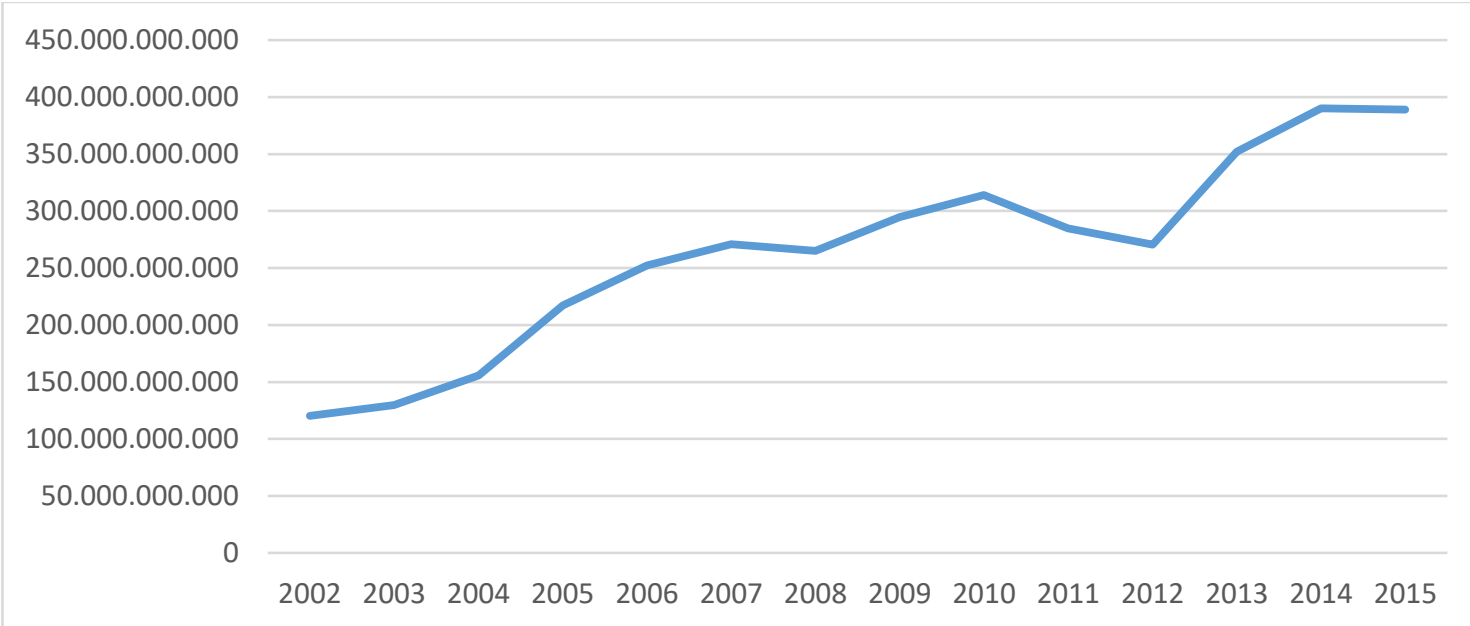

Fuente: Elaborado por el autor con base en información de las fichas técnicas del sistema de Información Hospitalario del Ministerio de Salud y Protección Social a en millones de pesos constantes de 2015

Dado que los ingresos se encuentran debidamente deflactados a precios de 2015, el crecimiento de los ingresos es real y se aproxima al $8 \%$ anual, por ajustes reales de los precios y por un incremento en los servicios facturados.

Figura 7 Ingresos Vs. Pasivos I Nivel 


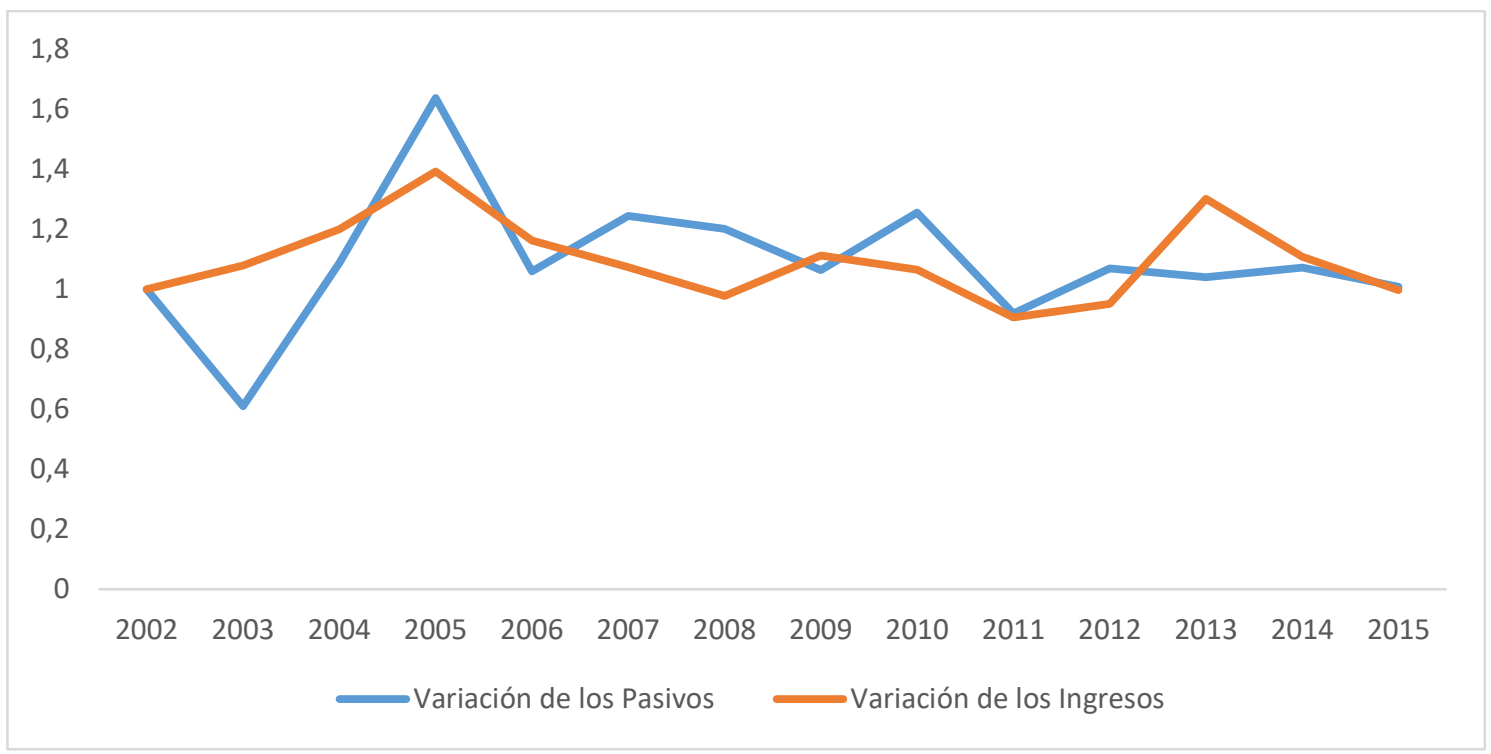

Fuente: Elaborado por el autor con base en información de las fichas técnicas del sistema de Información Hospitalario del Ministerio de Salud y Protección Social

La figura anterior explica que en la medida en que aumentan las ventas, los pasivos también lo hacen, a excepción del año 2013 cuando se recibieron recursos en los Hospitales que adoptaron un programa de saneamiento fiscal y financiero que permite un incremento de la operación sin afectación de los pasivos, debido a que se utilizaron recursos de cuentas maestras, previo a una adopción de medidas del programa de saneamiento fiscal y financiero indicado en la ley 1438 de 2011.

La cartera agrupada del primer nivel en millones de pesos es la siguiente:

\subsubsection{Cartera I Nivel}

Figura 8 Evolución de la cartera I Nivel

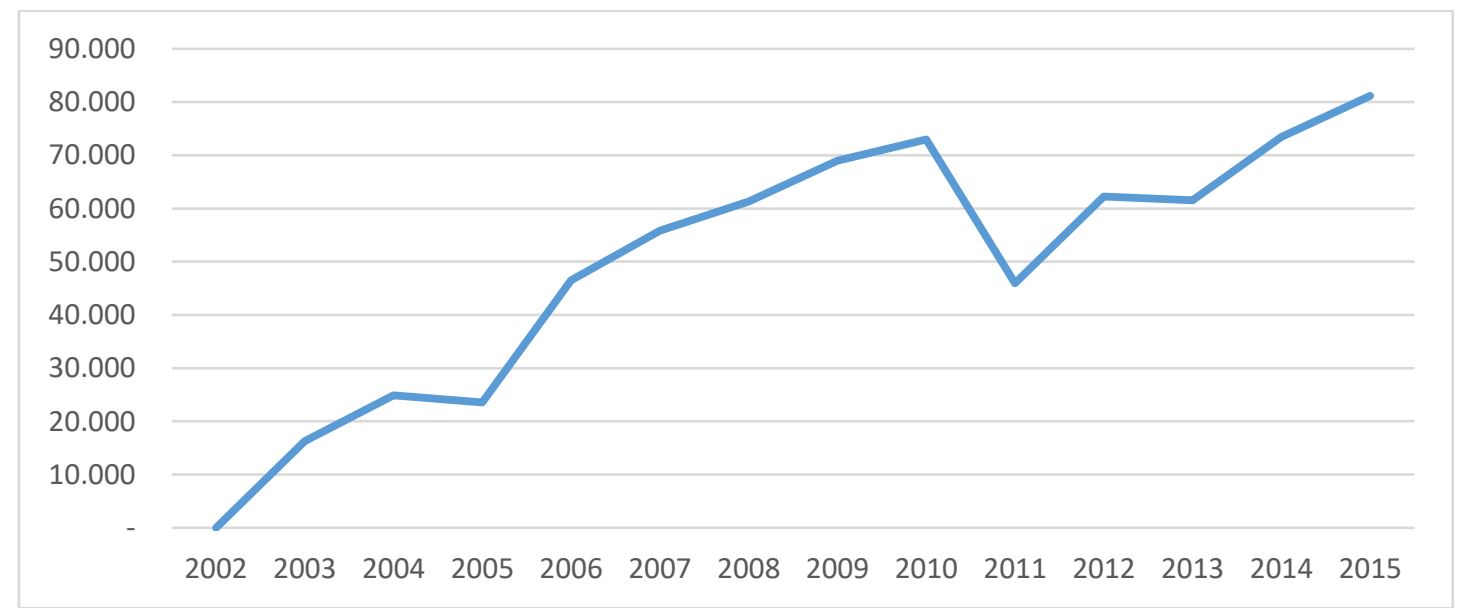

Fuente: Elaborado por el autor con base en información de las fichas técnicas del sistema de Información Hospitalario del Ministerio de Salud y Protección Social en millones de pesos constantes de 2015 
Las dificultades manifiestas en la operación de los hospitales de primer nivel son consecuencia de la detención del flujo de los recursos por parte de las EPS, la figura anterior es desarrollada a precios constantes de 2015 y se puede apreciar como de 2011 a 2015 pasa de poco más de $\$ 40$ mil millones a más de $\$ 80$ mil millones.

El crecimiento de la cartera por unidad hospitalaria de primer nivel se aprecia en la siguiente figura.

\section{Figura 9 Cartera por unidad hospitalaria primer nivel}

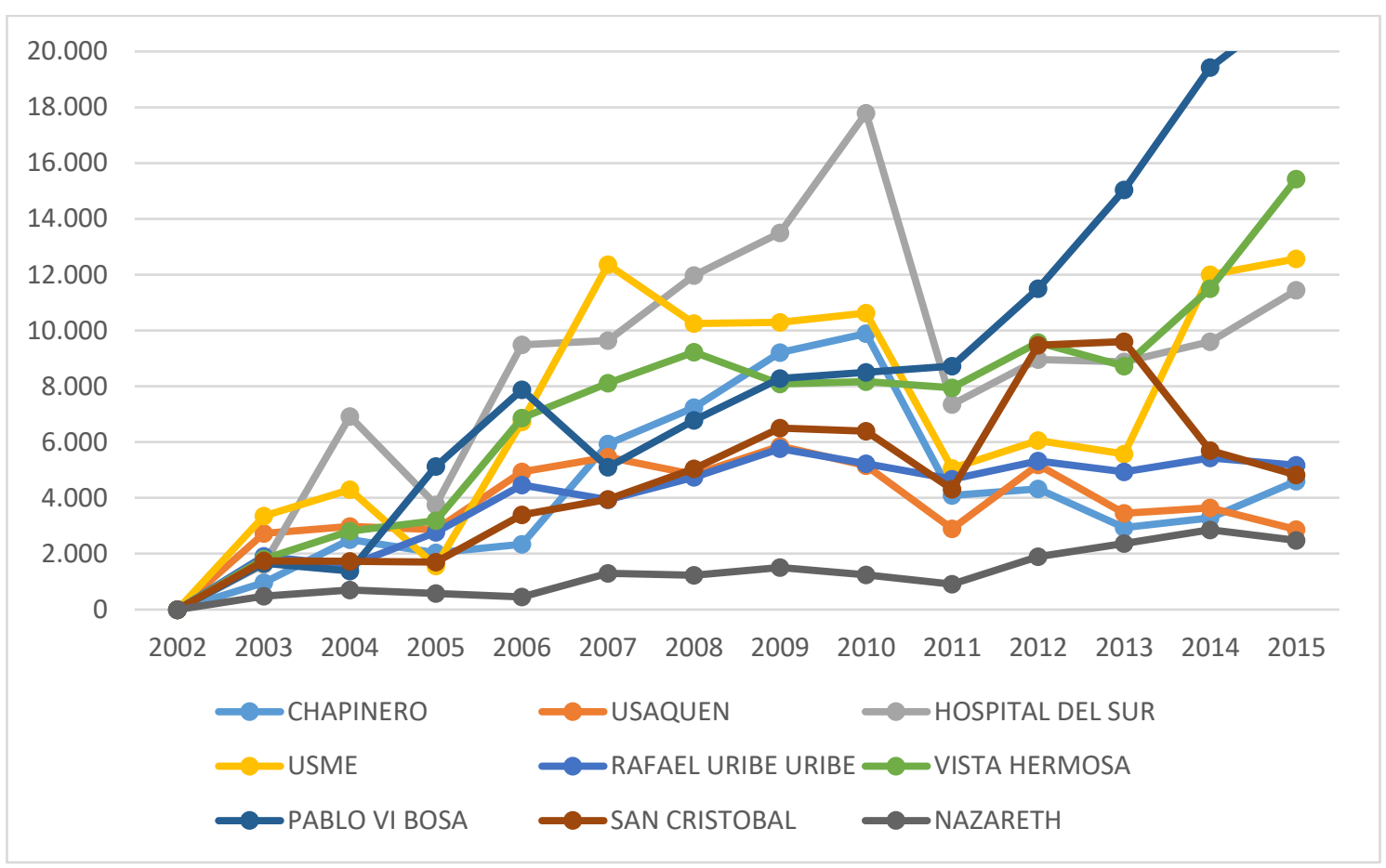

Fuente: Elaborado por el autor con base en información de las fichas técnicas del sistema de Información Hospitalario del Ministerio de Salud y Protección Social en millones de pesos constantes de 2015

El problema de deterioro de la cartera se explica en la demora de los pagos de EPS, de amplio conocimiento por parte del público, y luego de la mora algunas se han declarado en quiebra como Humana Vivir, Salud Total, Solsalud, Selvasalud y SaludCoop entre muchas (enumeradas en la tabla anterior) que no han retornado los recursos esperados por la prestación de los servicios a las IPS, con el agravante de que en las garantías de acceso a la salud, aun conociendo previamente la situación de las EPS, mediante la Ley 1438 no se puede negar el servicio de urgencias a los afiliados, que por supuesto, no tienen culpa de los problemas administrativos y en cambio grandes necesidades de cobertura. 
En un negocio, la velocidad de la recuperación de la inversión de recursos mediante recuperación o retorno se surte a través de las ventas (Goldratt \& Cox, 1998) en un circulo virtuoso que permite pagar los gastos de ventas y determinar las utilidades generadas por la operación. En los hospitales públicos, no se ha podido consolidar un circulo virtuoso, debido a que en un alto porcentaje la venta de servicios se realiza a crédito y las EPS dilatan el proceso de reconocimiento y pago que termina afectando el comportamiento del estado de resultados y el comportamiento de la ejecución presupuestal.

\subsubsection{Nivel}

El II Nivel de atención enfrenta una mayor demanda de servicios por cuanto los usuarios identifican que en estos centros por la preparación intelectual y disposición de infraestructura son resolutivos y no preventivos como pasa con la medicina familiar del primer nivel. Esta presión de la demanda también se visualiza en el comportamiento de los indicadores como se presenta a continuación.

\subsubsection{Pasivos II Nivel}

Los pasivos de los hospitales públicos de II nivel aumentaron a niveles que comprometían la operación, por cuanto los proveedores miraban con desconfianza la posibilidad de apalancar la operación al punto de exigir pagos por adelantado o en su defecto contra entrega. El capital de trabajo requerido en cualquier institución exige que las necesidades de corto plazo se apalanquen con recursos de corto plazo; si los proveedores no sienten seguridad en la transacción en últimas el precio de material médico y medicamentos se encarece elevando el estrés sobre los estados financieros.

Figura 10 Evolución de los pasivos del II Nivel de Atención

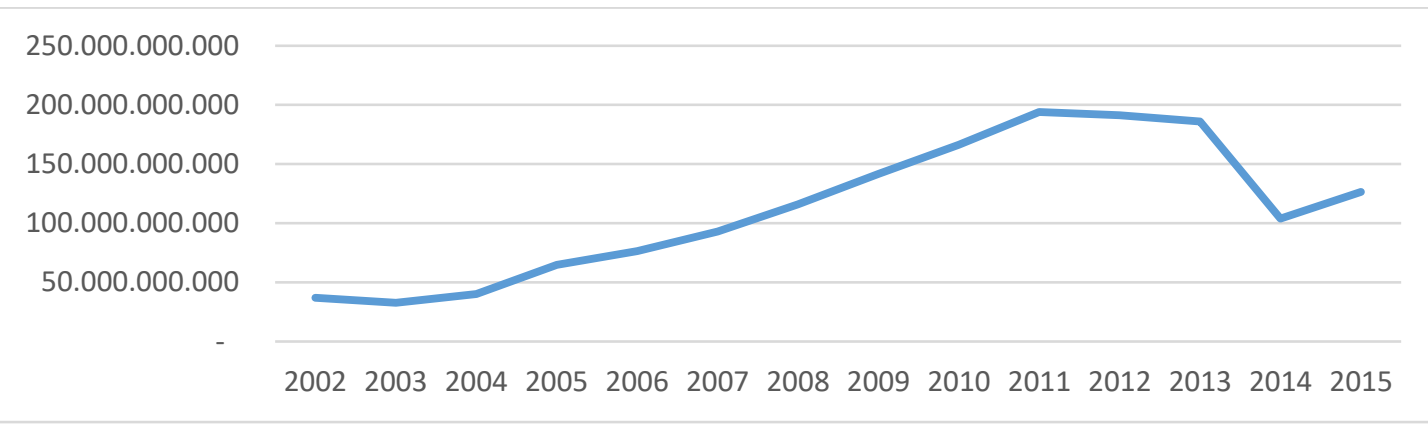

Fuente: Elaborado por el autor con base en información de las fichas técnicas del sistema de Información Hospitalario del Ministerio de Salud y Protección Social en millones de pesos constantes de 2015 
Los pasivos de los hospitales disminuyeron de forma notoria de 2013 a 2014, la inyección de recursos de uso específico provino de la liberación del uso de las cuentas maestras para dedicación exclusiva de pago de pasivos y recursos de inversión en concordancia con la ley 1608 de 2013 por medio de la cual se adoptan medidas para mejorar la liquidez y el uso de los recursos del sector salud mediante la liberación controlada del uso de excedentes de cuentas maestras.

El capítulo II de la Resolución 3042 explica en qué consisten las cuentas maestras y para contextualizar a los lectores de este documento, se definen las cuentas maestras.

Los fondos de salud, de acuerdo con las competencias establecidas para las entidades territoriales en la Leyes 715 de 2001 y 1122 de 2007, estarán conformados por las siguientes subcuentas:

1. Subcuenta de Régimen Subsidiado de Salud

2. Subcuenta de prestación de servicios de salud en lo no cubierto con subsidios a la demanda.

3. Subcuenta de salud pública colectiva

4. Subcuenta de otros gastos en salud

Las dinámicas de aplicación de los recursos han generado excedentes que se han acumulado, por ejemplo, en la subcuenta de régimen subsidiado, para poder descongelar esos recursos fue necesario establecer prioridades relacionadas con las necesidades mas sentidas de los hospitales, ejercicio desarrollado por la Secretaría de Hacienda y la Secretaría de Salud.

En la siguiente figura se aprecia el impacto sobre los hospitales públicos de segundo nivel. 


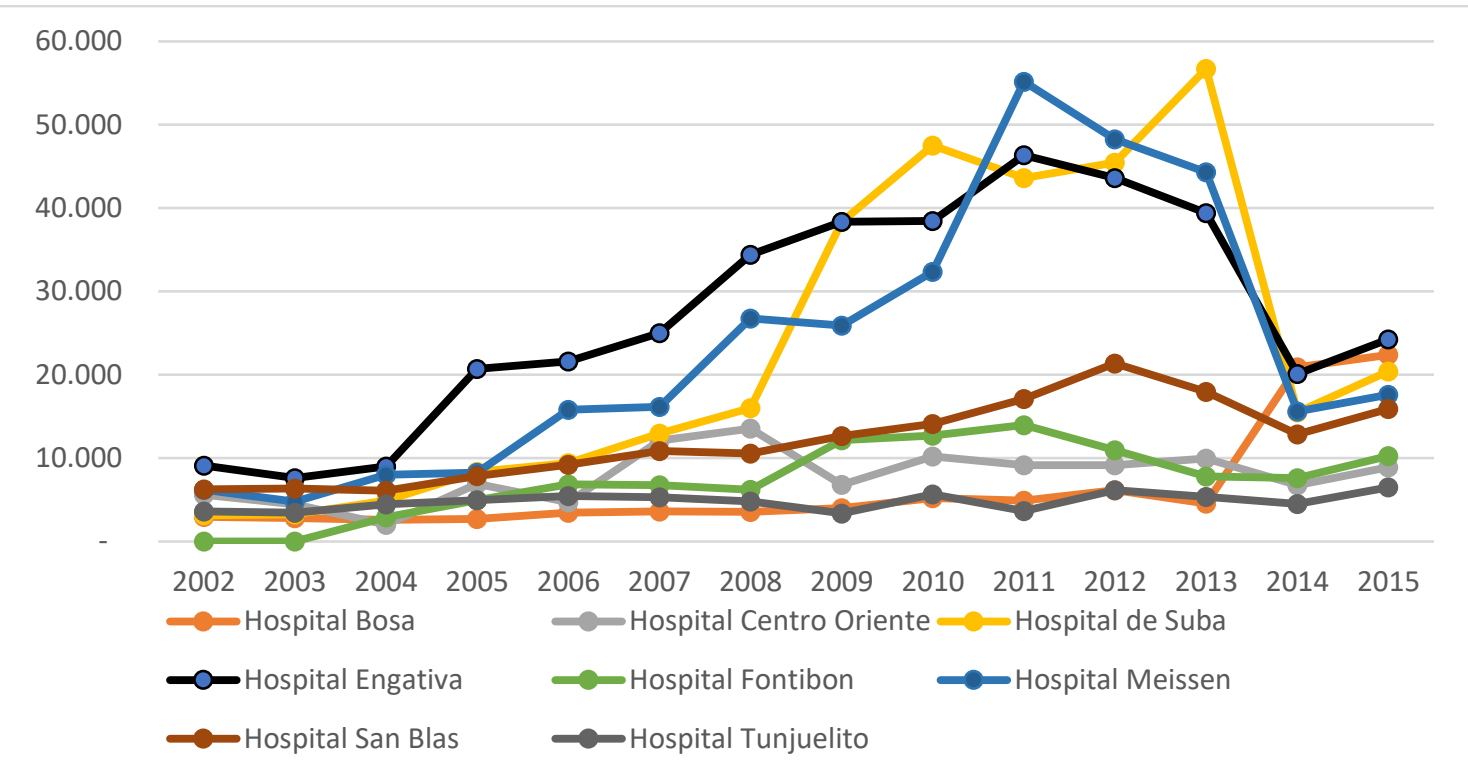

Fuente: Elaborado por el autor con base en información de las fichas técnicas del sistema de Información Hospitalario del Ministerio de Salud y Protección Social en millones de pesos constantes de 2015

Se aprecia que a partir de 2008 se presenta una explosión en el crecimiento de los pasivos, en particular para los Hospitales de Suba, Engativá y Meissen, al ser declarados en riesgo alto, los giros de las cuentas maestras hacen que se de una convergencia sobre los niveles de endeudamiento para el 2015.

\subsubsection{Ingresos II Nivel}

Los hospitales de segundo nivel alcanzaron las metas de producción en UVR que se habían propuesto luego de los programas de saneamiento fiscal y financiero, sin embargo los ingresos no aumentaron, la razón principal es la cesación de pagos por parte de la EPS CAPRECOM y la reducción del porcentaje de pago de las demás EPS del $80 \%$ al $50 \%$ a partir del I semestre de 2015 (Secretaría Distrital de Salud, 2015), esta situación afecta de manera directa por cuanto los ingresos del II y III Nivel de atención dependen de los pagos de las EPS.

El comportamiento de los ingresos es el siguiente:

Figura 12 Ingresos II Nivel 


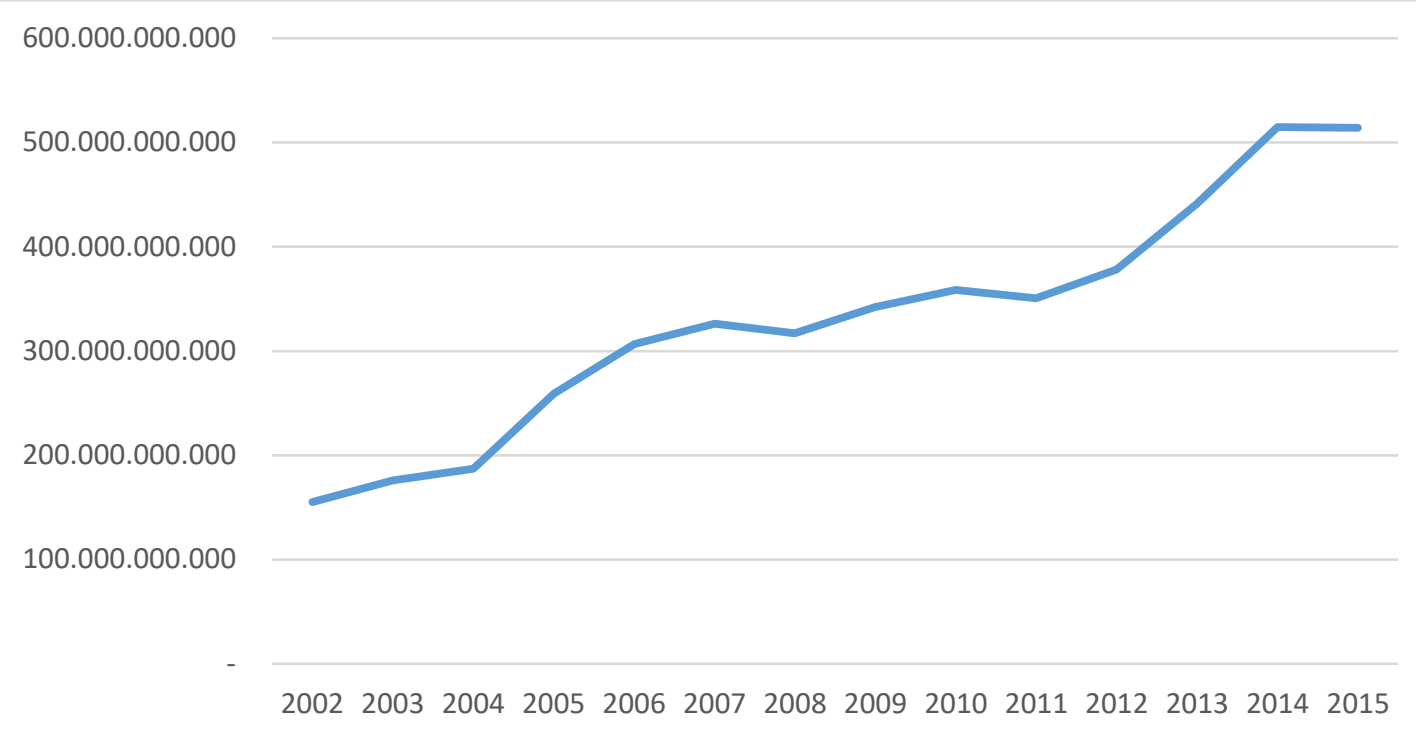

Fuente: Elaborado por el autor con base en información de las fichas técnicas del sistema de Información Hospitalario del Ministerio de Salud y Protección Social a precios constantes de 2015

\subsubsection{Cartera II Nivel}

Las diferentes unidades hospitalarias de II nivel han sufrido la disminución de los flujos derivados del pago de los ingresos reconocidos de los servicios de salud prestados, esto se refleja en el comportamiento de la cartera.

Figura 13 Evolución de la Cartera consolidada de los Hospitales de II Nivel

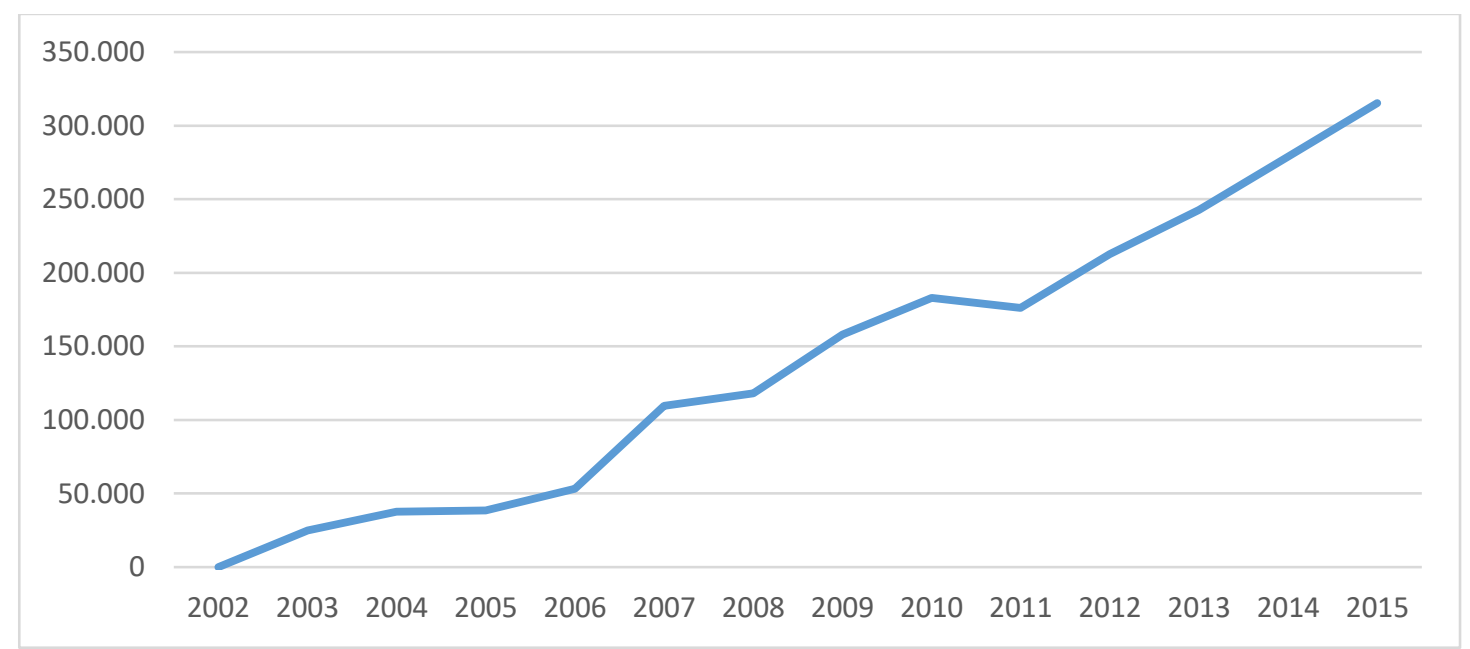

Fuente: Elaborado por el autor con base en información de las fichas técnicas del sistema de Información Hospitalario del Ministerio de Salud y Protección Social en millones de pesos constantes de 2015 
Suba, Meissen y Engativá son los Hospitales con mayor afectación como se puede apreciar en la siguiente figura.

Figura 14 Evolución de la Cartera de los hospitales de II Nivel

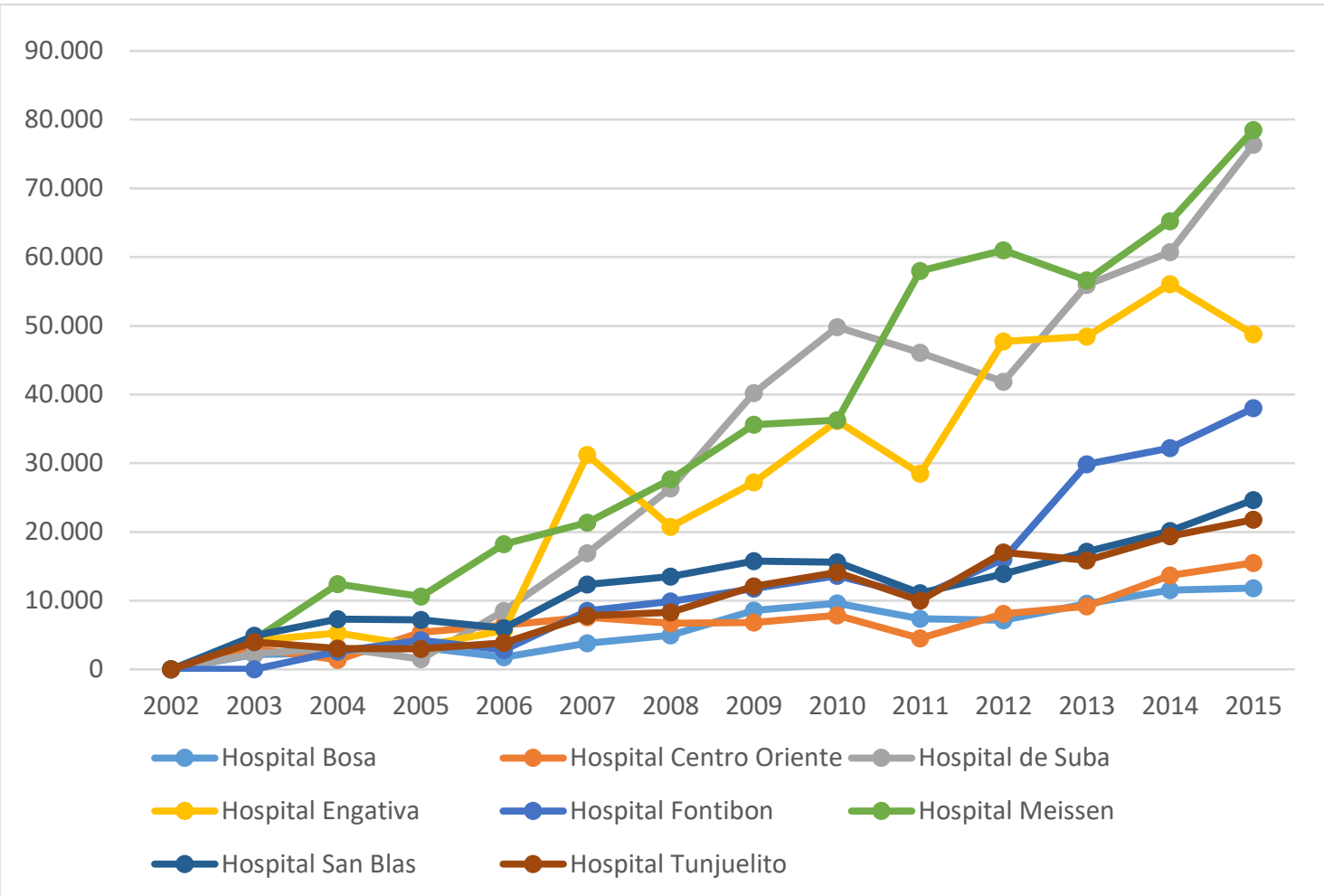

Fuente: Elaborado por el autor con base en información de las fichas técnicas del sistema de Información Hospitalario del Ministerio de Salud y Protección Social en millones de pesos constantes de 2015

Las características de los hospitales de II nivel son bastante heterogéneas, situación que dificulta las comparaciones. Algunos hospitales de II nivel cuentan con centros de atención satelitales como los hospitales de I nivel y han tenido contratos del Plan de Intervenciones Colectivas con el Distrito.

\subsubsection{Nivel}

\subsubsection{Pasivos III Nivel}

Los pasivos de los hospitales fueron sometidos a dos tratamientos; el primero la revisión de las acreencias reales, que para el caso de los hospitales que se calificaron en riesgo, recibieron recursos por transferencia para honrar sus deudas, y la segunda el plan de convergencia a NIIF (Normas Internacionales de Información Financiera) que instó a las Instituciones a depurar las cuentas, con efectos de reducción sobre las cifras que se pueden considerar más ajustadas a la realidad. 
En forma consecuente con el crecimiento de la cartera, que hace parte de los activos de los Hospitales, crecen los pasivos cuando no llegan los recursos de forma oportuna y que para poder operar requieren acudir a un mayor número de intermediarios que encarecen la operación hospitalaria porque los precios de intermediación son cada vez más altos con el fin de mitigar los costos asociados al riesgo y a la demora de los pagos.

Figura 15 evolución de los pasivos consolidados de los hospitales de III Nivel

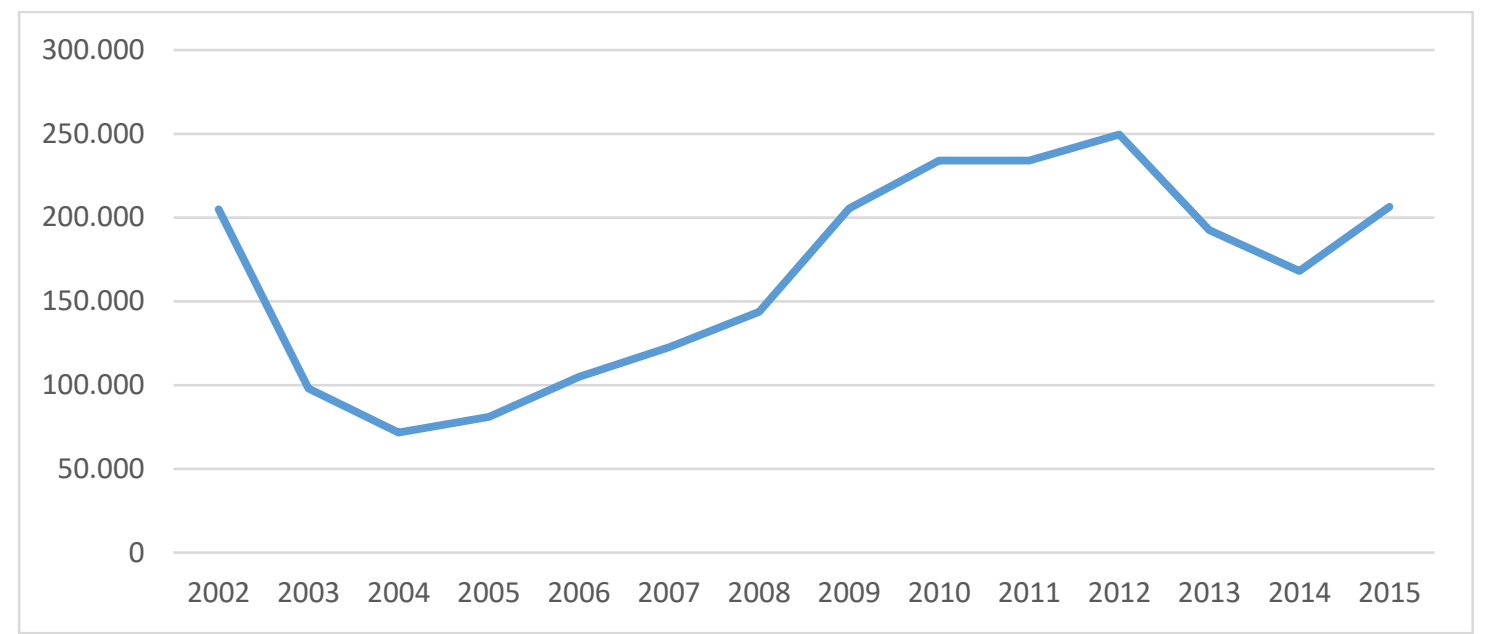

Fuente: Elaborado por el autor con base en información de las fichas técnicas del sistema de Información Hospitalario del Ministerio de Salud y Protección Social en millones de pesos constantes de 2015

\subsubsection{Ingresos III Nivel}

El comportamiento de los ingresos del III nivel es el siguiente:

No obstante que no se aprecian incrementos importantes en el número de camas en el tercer nivel, si se aprecia un incremento de los ingresos. Esta situación que no 
Figura 16 Ingresos por unidad hospitalaria III Nivel

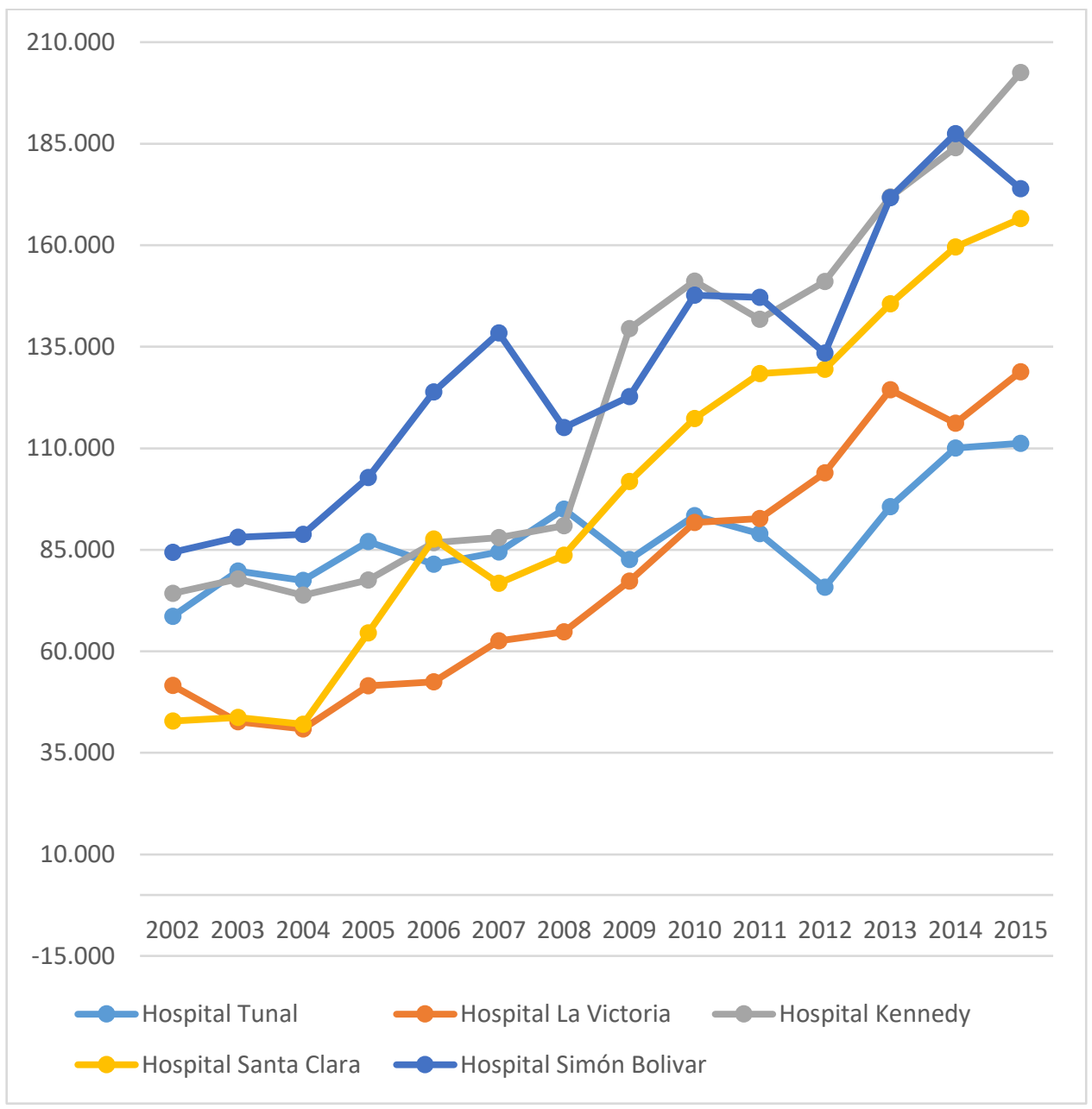

Fuente: Elaborado por el autor con base en información de las fichas técnicas del sistema de Información Hospitalario del Ministerio de Salud y Protección Social en millones de pesos constantes de 2015

El hospital de mayores ingresos en el III Nivel es el Hospital de Kennedy seguido por el Simón Bolívar, los cuales son referentes de varios tratamientos únicos, como la unidad de quemados y cirugía plástica y otros tratamientos de alto costo, como cardiología pediátrica, cirugía pediátrica, y salud mental entre otros que lo convierten a estos hospitales en referentes con gran reconocimiento en el distrito.

\subsubsection{Cartera III Nivel}

Figura 17 Evolución de la cartera consolidada de los hospitales de III nivel 


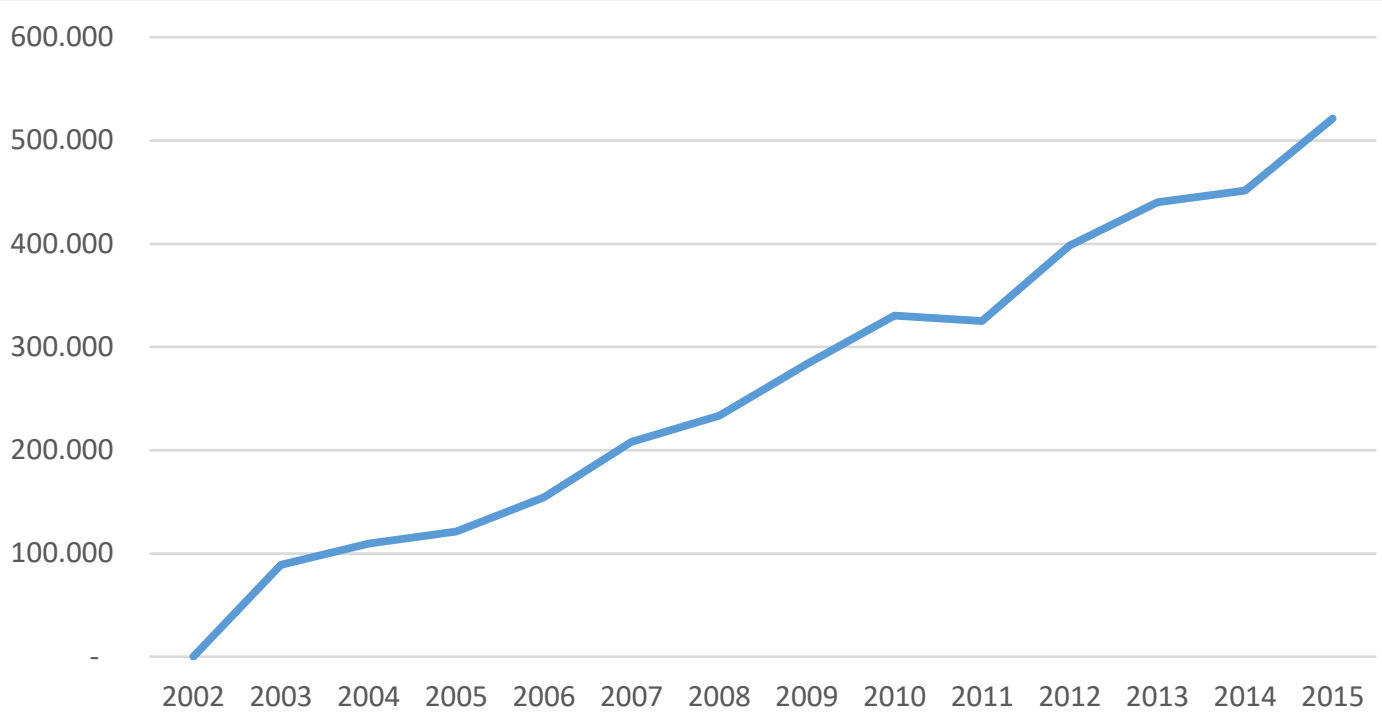

Fuente: Elaborado por el autor con base en información de las fichas técnicas del sistema de Información Hospitalario del Ministerio de Salud y Protección Social en millones de pesos constantes de 2015

La cartera de los Hospitales de III Nivel se ve disparada en la gráfica anterior, el crecimiento medio de la cartera es superior al $20 \%$ anual en términos reales, lo que debe interpretar como una tasa explosiva y de difícil manejo para las administraciones de turno.

Figura 18 Evolución de la cartera por unidad hospitalaria de III Nivel

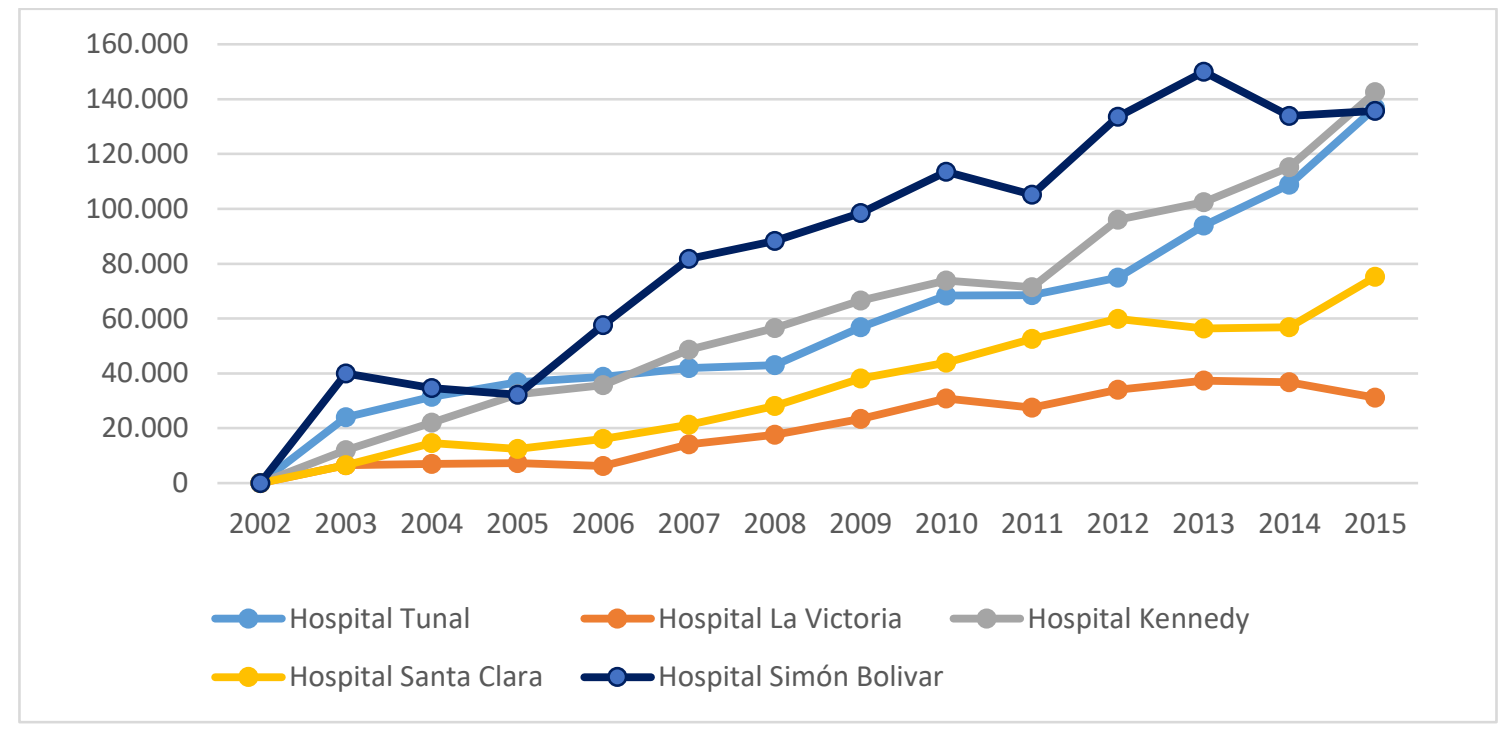

Fuente: Elaborado por el autor con base en información de las fichas técnicas del sistema de Información

Hospitalario del Ministerio de Salud y Protección Social en millones de pesos constantes de 2015

En términos individuales el Hospital que más factura, también resulta ser el más castigado con el crecimiento de su cartera, este es el Hospital Simón Bolívar, sin considerar que el crecimiento de la cartera de las demás IPS sea aceptable. 


\subsubsection{Pasivos por Niveles}

El peso del crecimiento de las acreencias es diferente por niveles de atención a precios constantes de 2015, el mismo se aprecia en la siguiente figura.

Figura 19 Evolución de los pasivos consolidados por niveles de atención

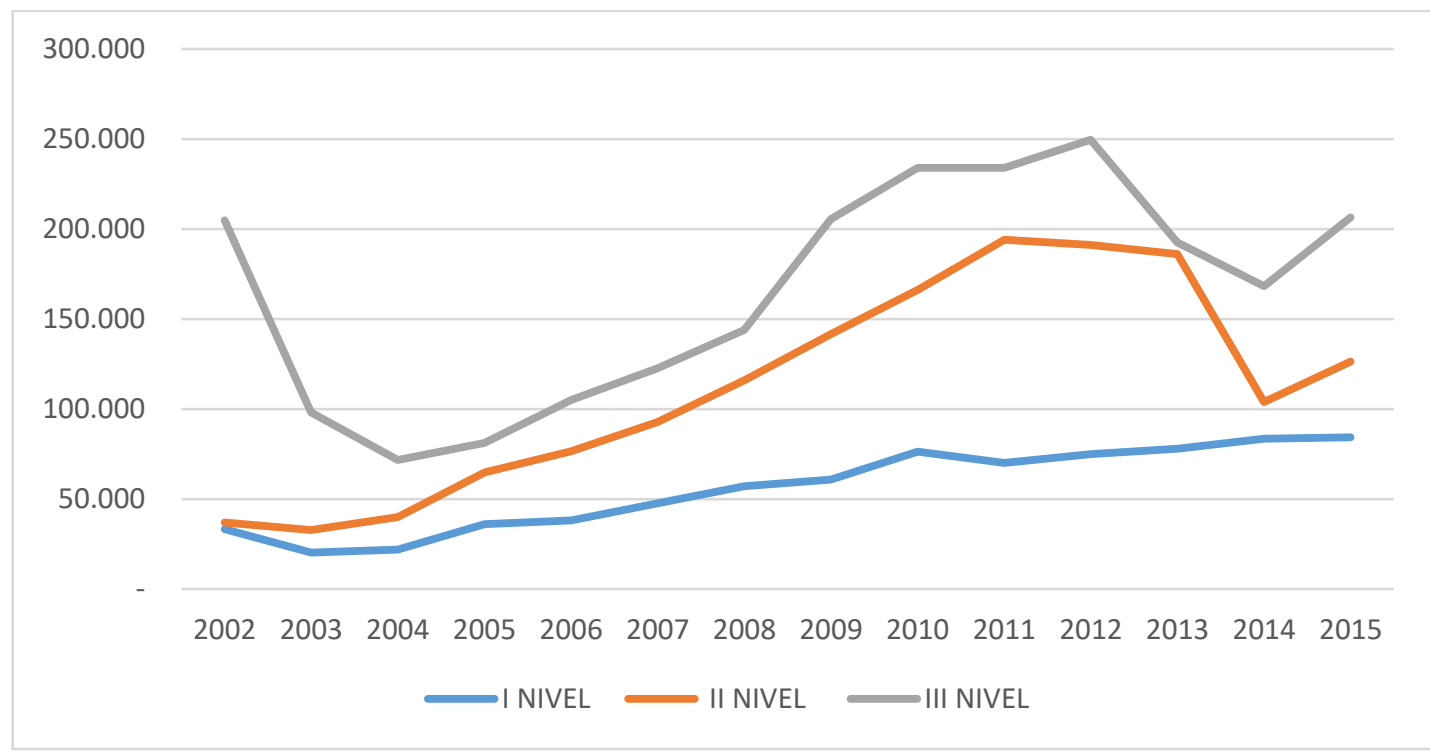

Fuente: Elaborado por el autor con base en información de las fichas técnicas del sistema de Información Hospitalario del Ministerio de Salud y Protección Social a precios constantes de 2015

Los pasivos se mantienen gracias a la posibilidad de uso de los recursos de cuentas maestras que se implementó por medio de los Programas de Saneamiento Fiscal y Financiero. Sin embargo, el crecimiento de la cartera a precios constantes de 2015 es elocuente, tal como se muestra en la siguiente figura. 
La composición de los pasivos por niveles de atención en la siguiente:

Figura 20 Participación por niveles de los pasivos totales

\section{Composición de los pasivos}

TOTAL: 416.927

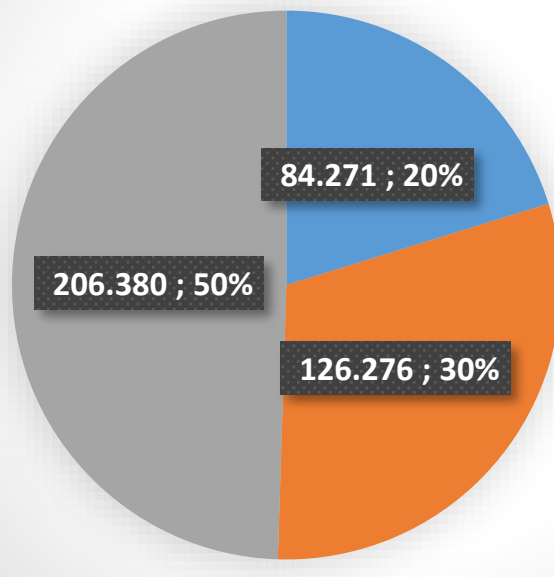

Fuente: Elaborado por el autor con base en información de las fichas técnicas del sistema de Información Hospitalario del Ministerio de Salud y Protección Social en millones de pesos de 2015

\subsubsection{Cartera por Niveles}

\section{Cartera Total}

Figura 21 Evolución de la cartera total de los hospitales de Bogotá

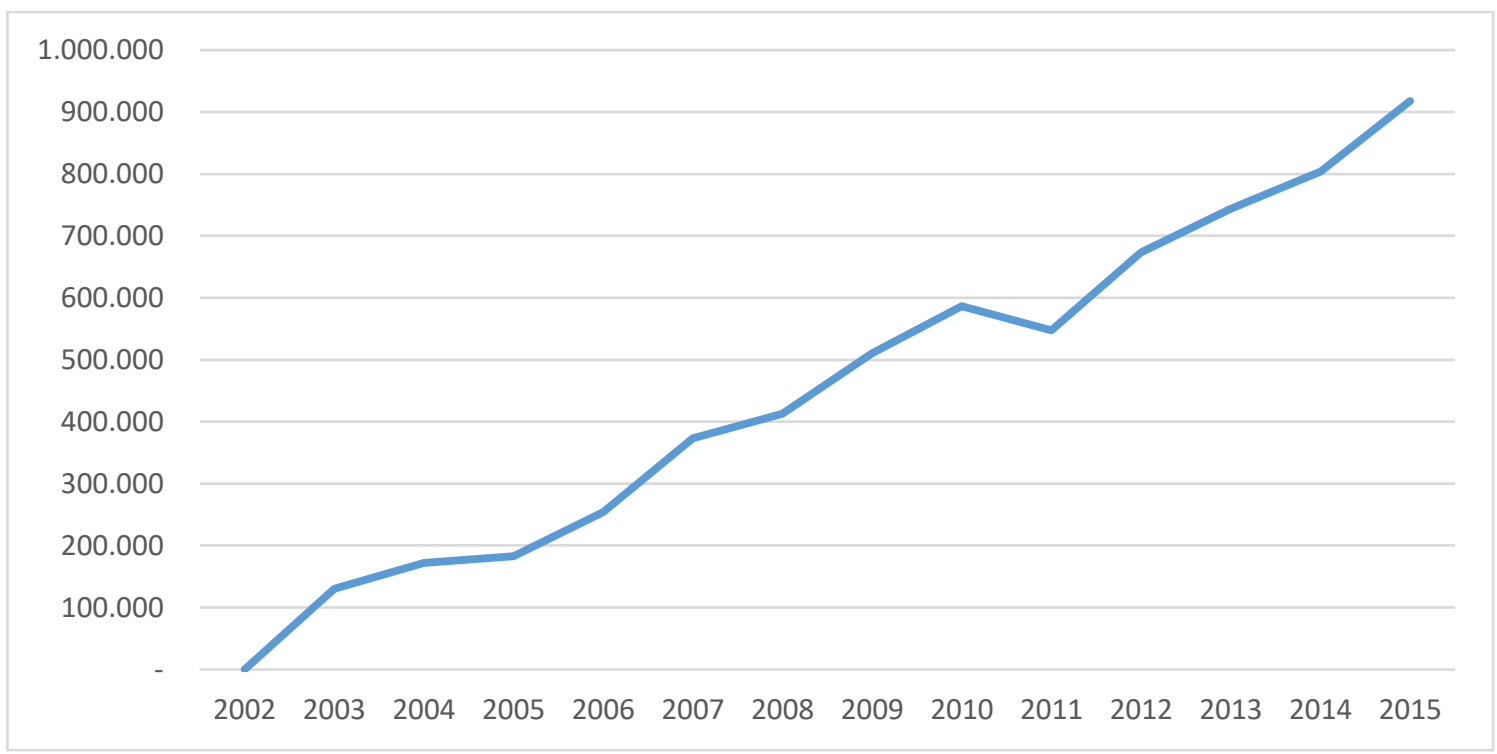

Fuente: Elaborado por el autor con base en información de las fichas técnicas del sistema de Información Hospitalario del Ministerio de Salud y Protección Social en millones de pesos de 2015 
Figura 22 Evolución de la cartera en los tres niveles de atención

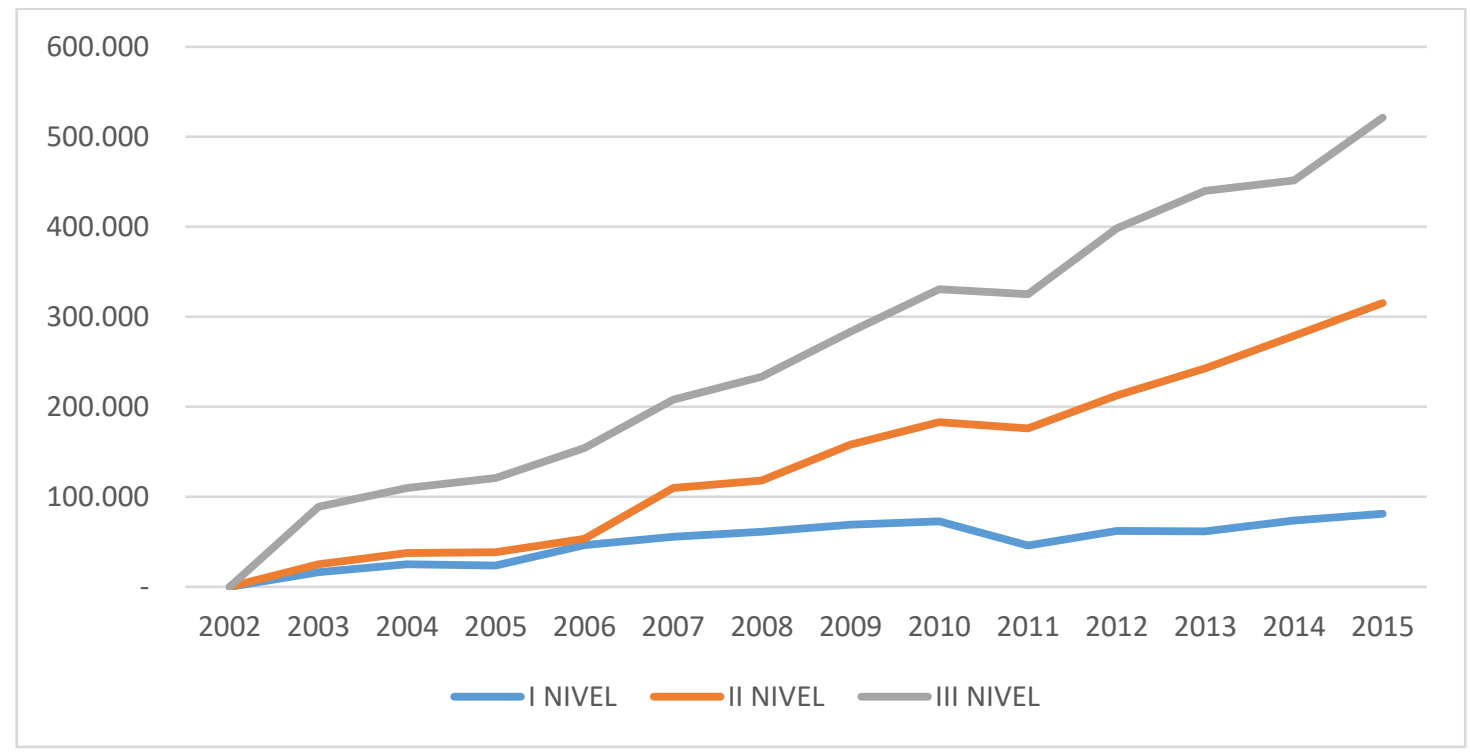

Fuente: Elaborado por el autor con base en información de las fichas técnicas del sistema de Información Hospitalario del Ministerio de Salud y Protección Social en millones de pesos de 2015

Esto significa que la cartera no para de crecer mientras que los Hospitales hacen esfuerzos ingentes por contener los pasivos, y en los casos en los que los que han sido calificados con RA o medio han recibido recursos del ente territorial que disminuyeron la presión de los acreedores por cuanto se pagaron pasivos exigibles de vigencias anteriores a la calificación de riesgo con un propósito claro de mantener la actividad de los hospitales. La composición de la cartera por niveles de atención es la siguiente:

Figura 23 Composición de la cartera por niveles

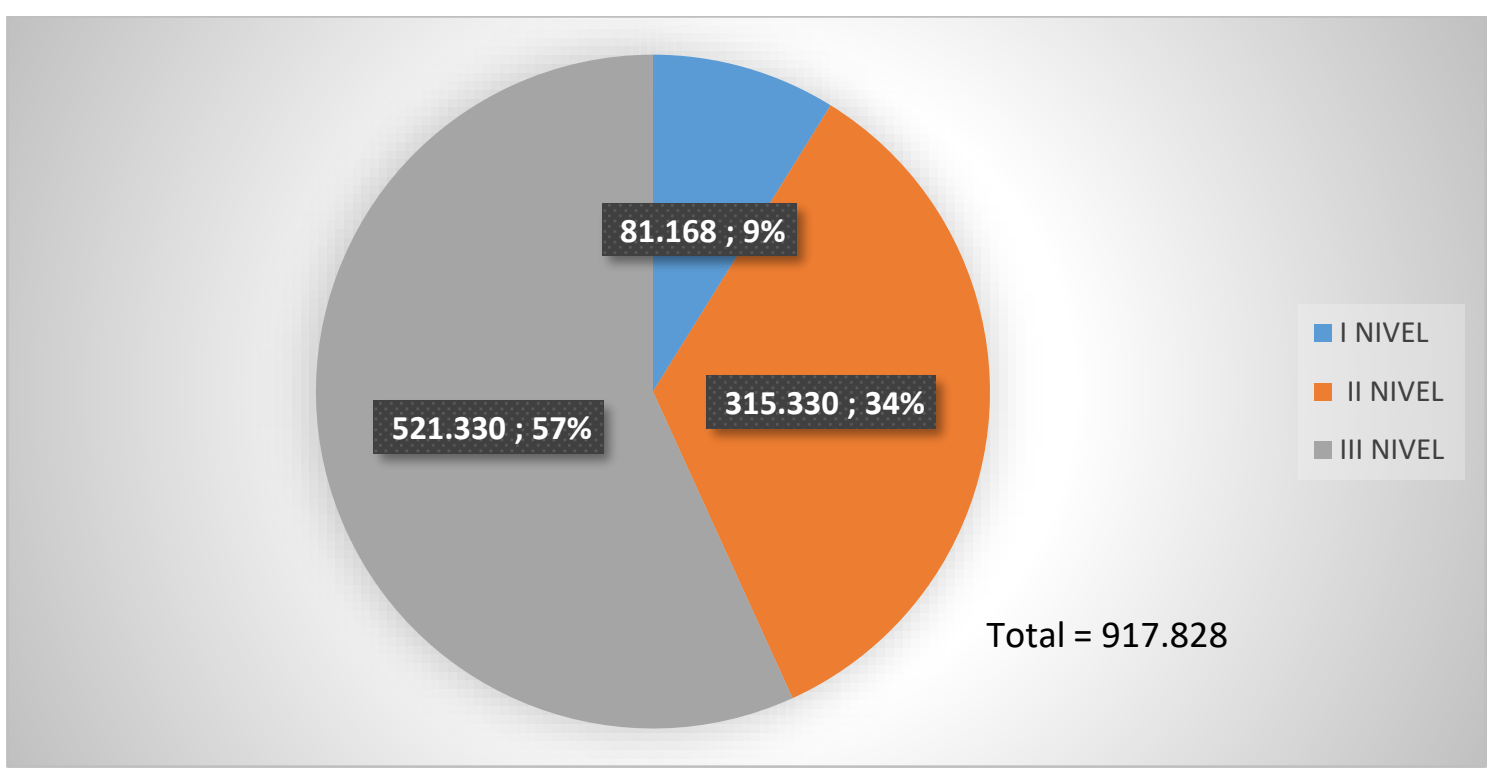

Fuente: Elaborado por el autor con base en información de las fichas técnicas del sistema de Información Hospitalario del Ministerio de Salud y Protección Social en millones de pesos de 2015 


\subsection{Aspectos Gestión Hospitalaria}

Una forma de establecer el comportamiento de los resultados, desde el punto de vista de gestión hospitalaria es revisar la producción de UVR (unidades de valor relativo de los hospitales) en las que el Ministerio de Salud y Protección Social ha invertido capital intelectual con el fin de establecer un mecanismo que haga comparables los niveles de producción.

Las UVR son estimaciones que buscan homogenizar la producción de tal forma que la producción de servicios es multiplicada por un estimador que valora el uso de los recursos hospitalarios como son la cantidad y calidad de mano de obra para un procedimiento, el uso de las instalaciones y la tecnología, estos estimadores son proporcionados por el Ministerio de Salud y Protección Social y se adelantan por niveles de atención.

Los siguientes son las figuras de producción por UVR:

Figura 24 Producción por UVR primer nivel de atención

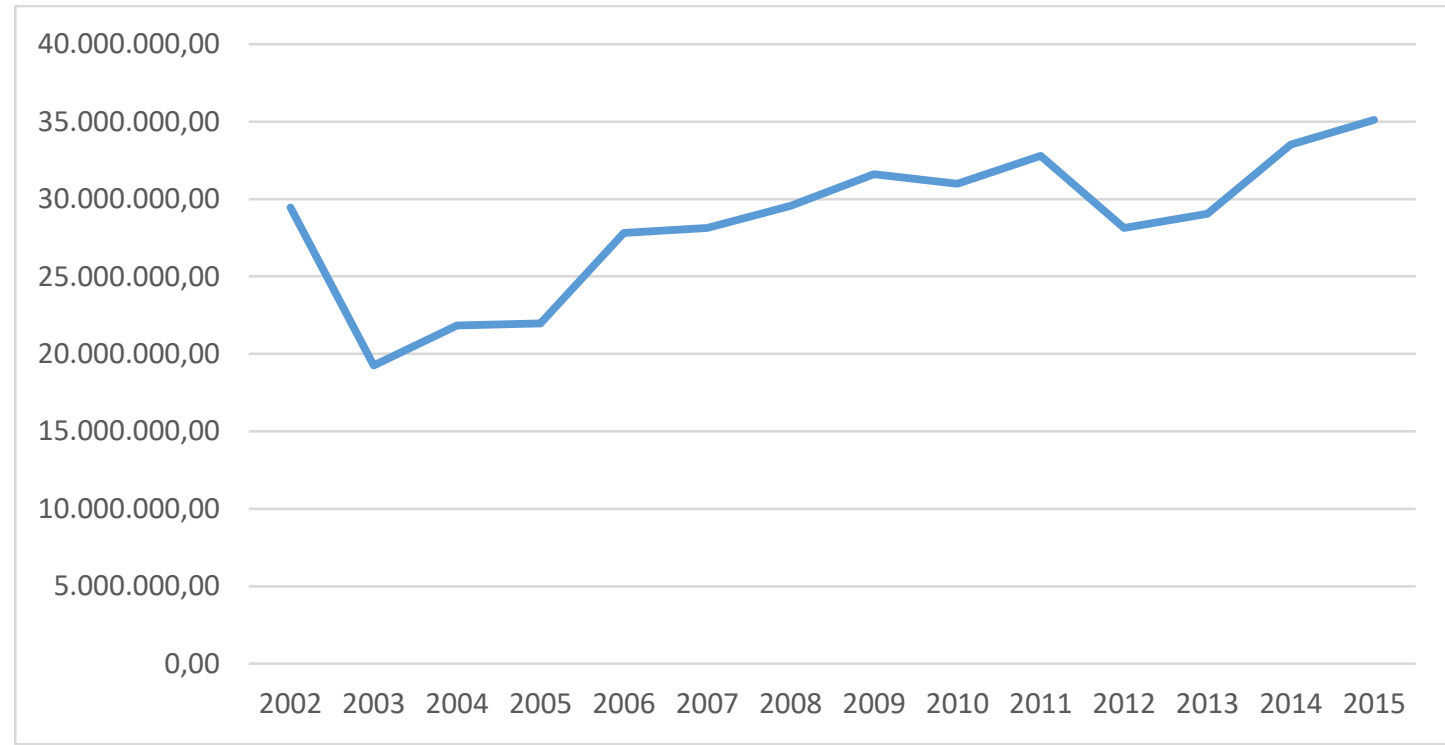

Fuente: Información tomada de la ficha técnica del Ministerio de Salud y Protección Social

Desde la perspectiva de la producción en el primer nivel se han atendido un mayor volumen de servicios, con un incremento anual medio de $1,1 \%$.

El desempeño de la producción de servicios hospitalarios respecto de la variación de los gastos, comportamientos de los ingresos 
Figura 25 Producción equivalente UVR por Hospital primer nivel

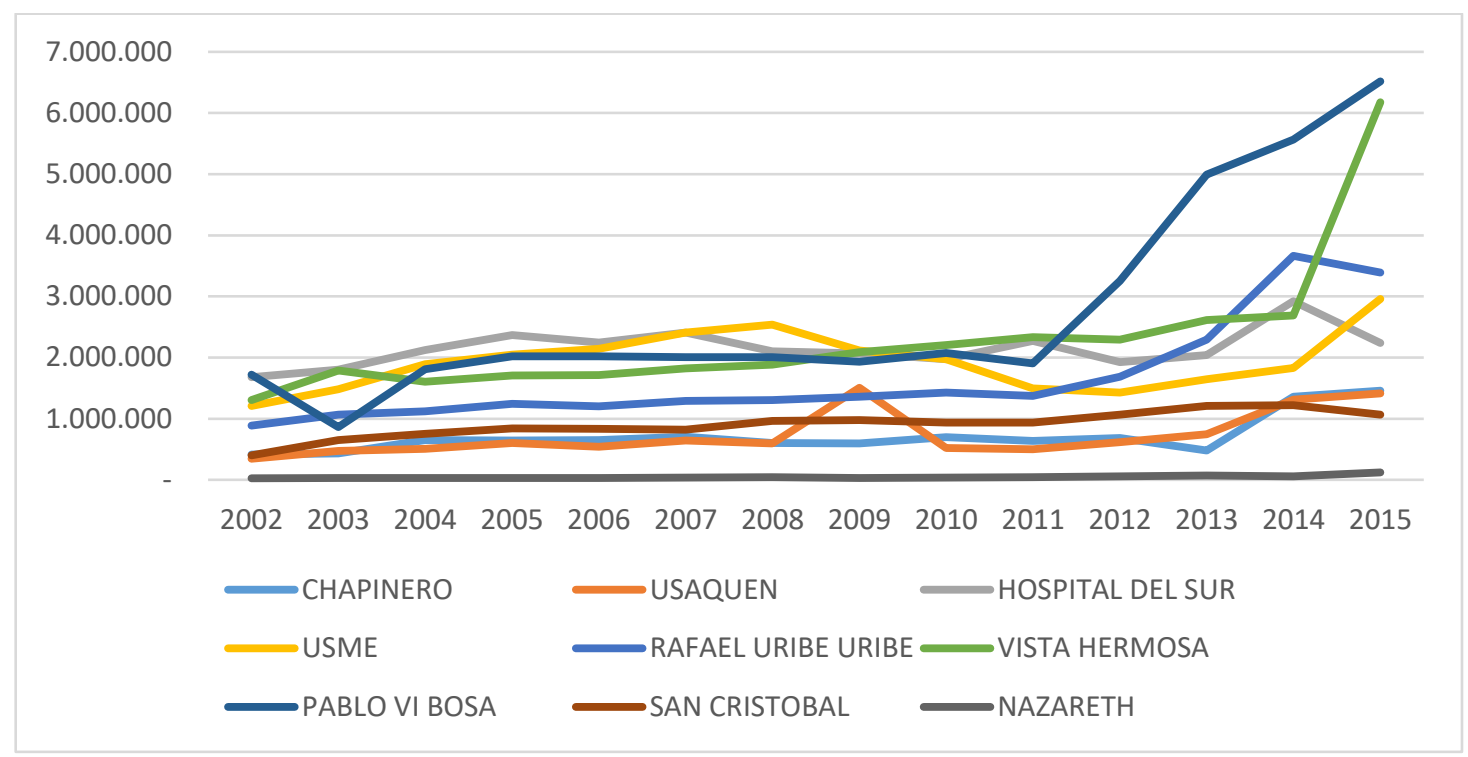

Fuente: Información tomada de la ficha técnica del Ministerio de Salud y Protección Social

Esto significa que, en el tiempo, para primer nivel no existe una presión importante de la demanda salvo en los hospitales Pablo VI Bosa y Vista Hermosa.

Figura 26 Producción UVR II Nivel

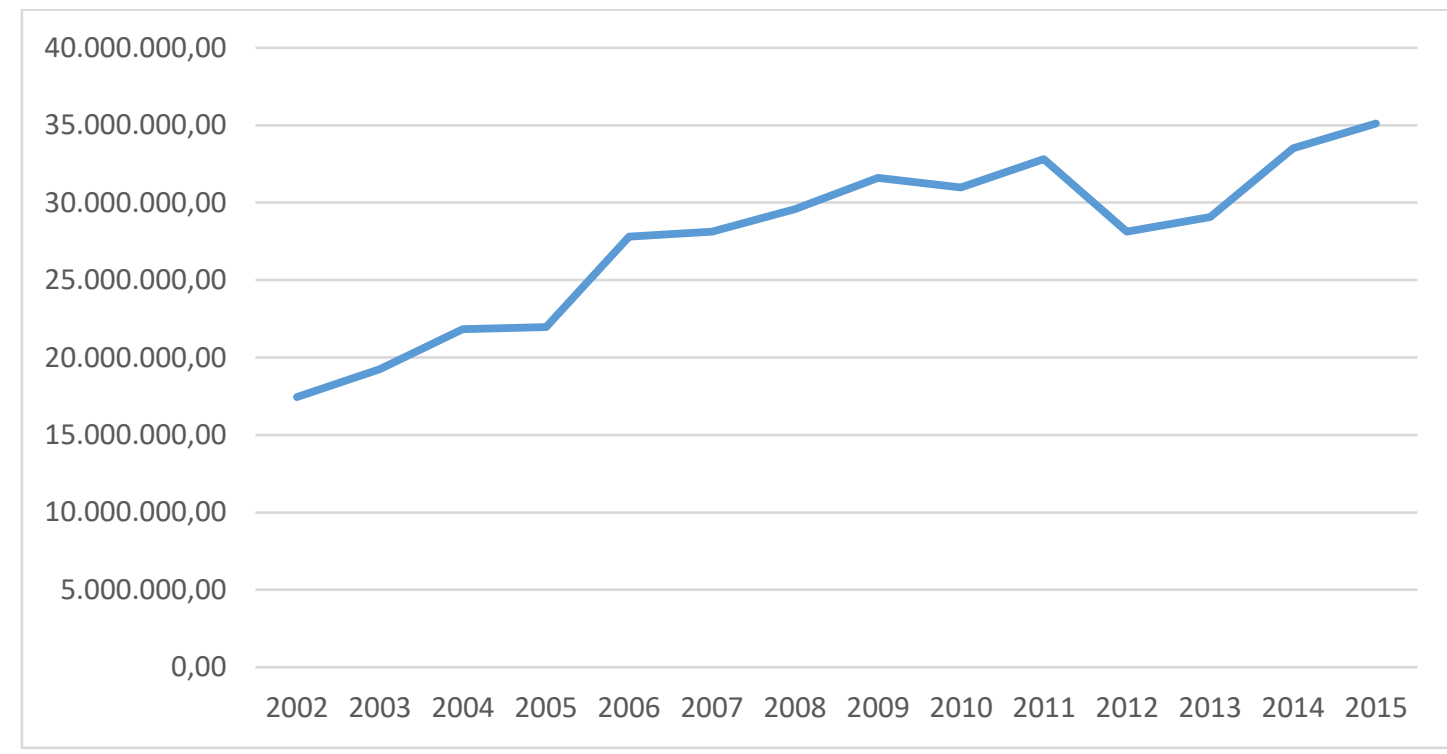

Fuente: Información tomada de la ficha técnica del Ministerio de Salud y Protección Social

Esto significa que en el II Nivel ha tenido un crecimiento medio de la demanda equivalente al 5,12\% anual y hasta este momento de las observaciones, ha recibido el peso del crecimiento de la demanda de servicios. 
Figura 27 Producción equivalente UVR por Hospital II Nivel

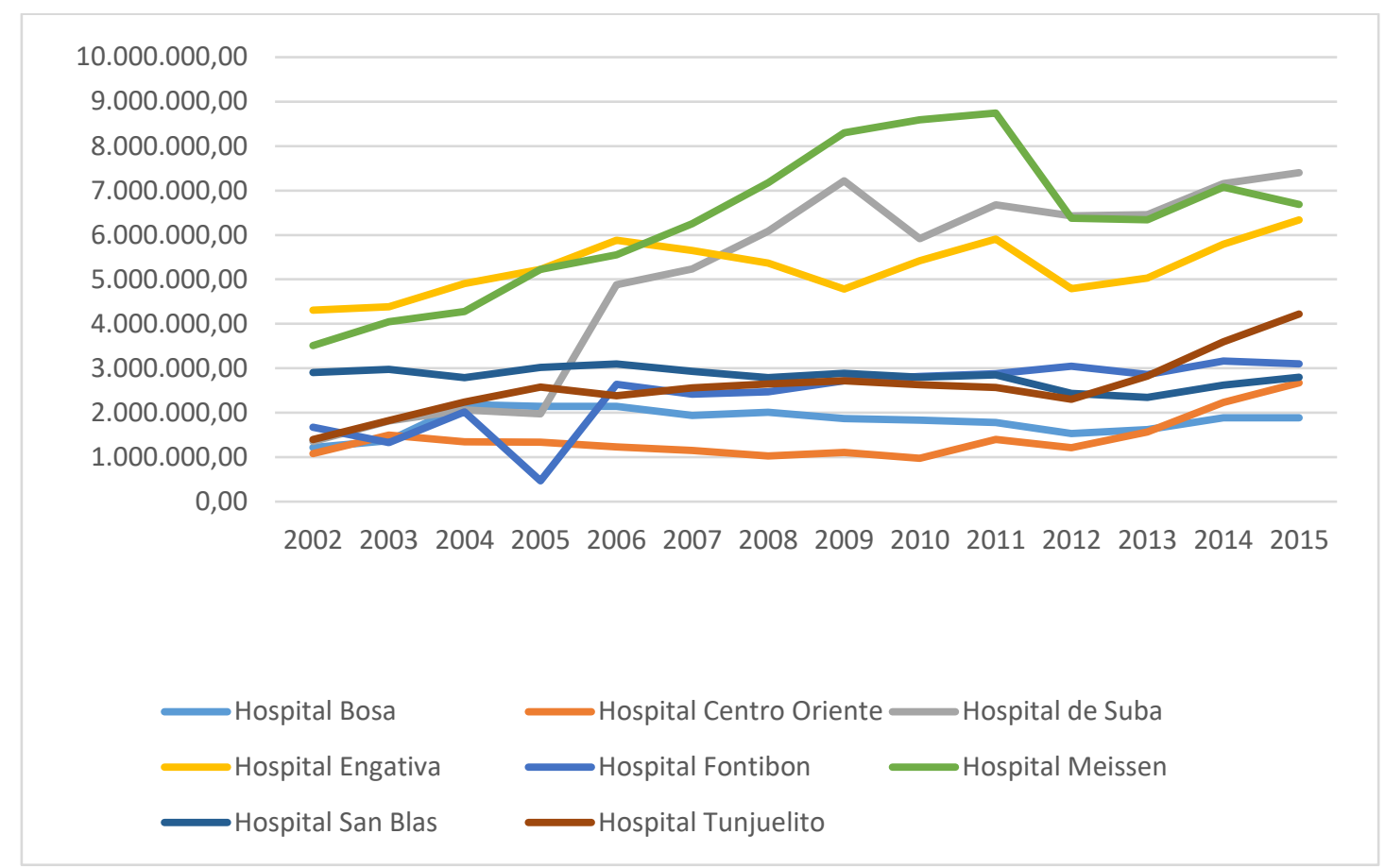

Fuente: Información tomada de la ficha técnica del Ministerio de Salud y Protección Social

Los niveles de producción del segundo nivel medido por unidades de valor relativo han presentado importantes variaciones en los Hospitales de Engativa, Suba y Meissen como efectos del comportamiento de la demanda por servicios de salud en estas importantes zonas de influencia. El Hospital Meissen II Nivel ESE presenta un comportamiento atípico al llegar cerca de los nueve millones de UVR durante el 2011 y caer hasta los seis millones de UVR durante el 2012; periodo sobre el cual se adelantan investigaciones relacionadas con el cartel de la contratación por diversos contratos millonarios de diversa índole, incluidos contratos con las EPS que provocaron una importante presión de la demanda y una explosión de oferta de servicios.

\section{Figura 28 Producción por UVR III Nivel}

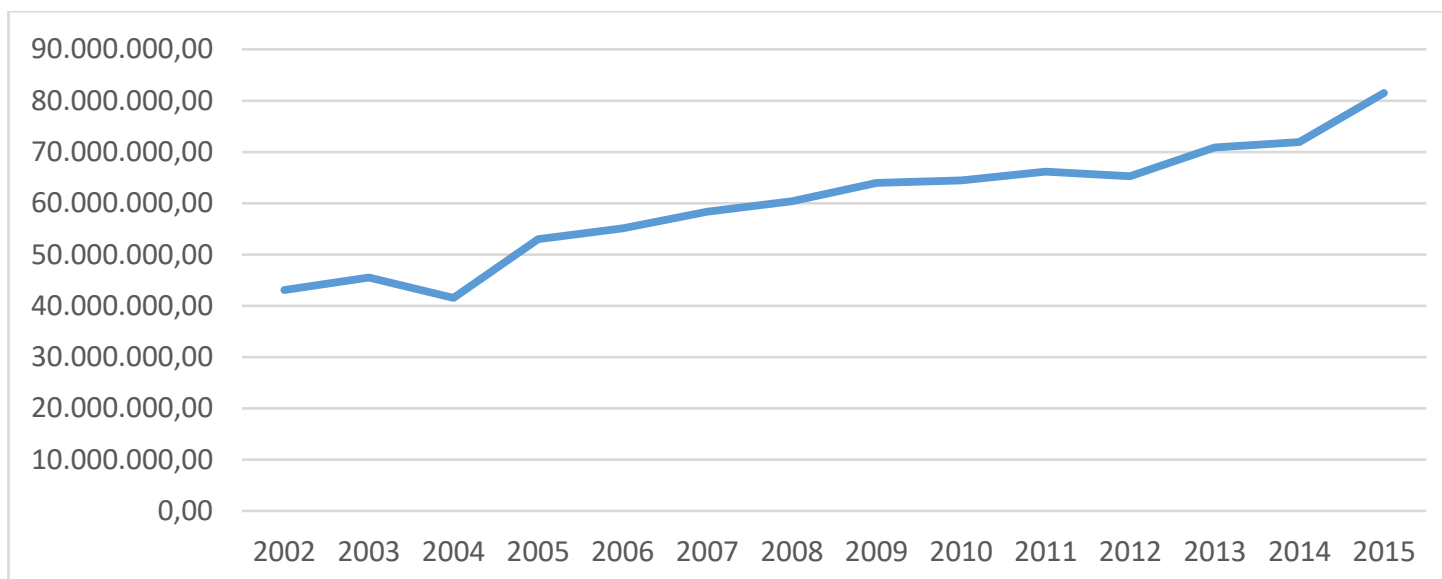

Fuente: Información tomada de la ficha técnica del Ministerio de Salud y Protección Social 
El III nivel ha atendido una presión de la demanda de servicios equivalente al 4,62\% en promedio en el periodo 2002-2015

La revisión del comportamiento individual de las unidades hospitalarias es la siguiente,

Figura 29 Producción equivalente UVR por Hospital III Nivel

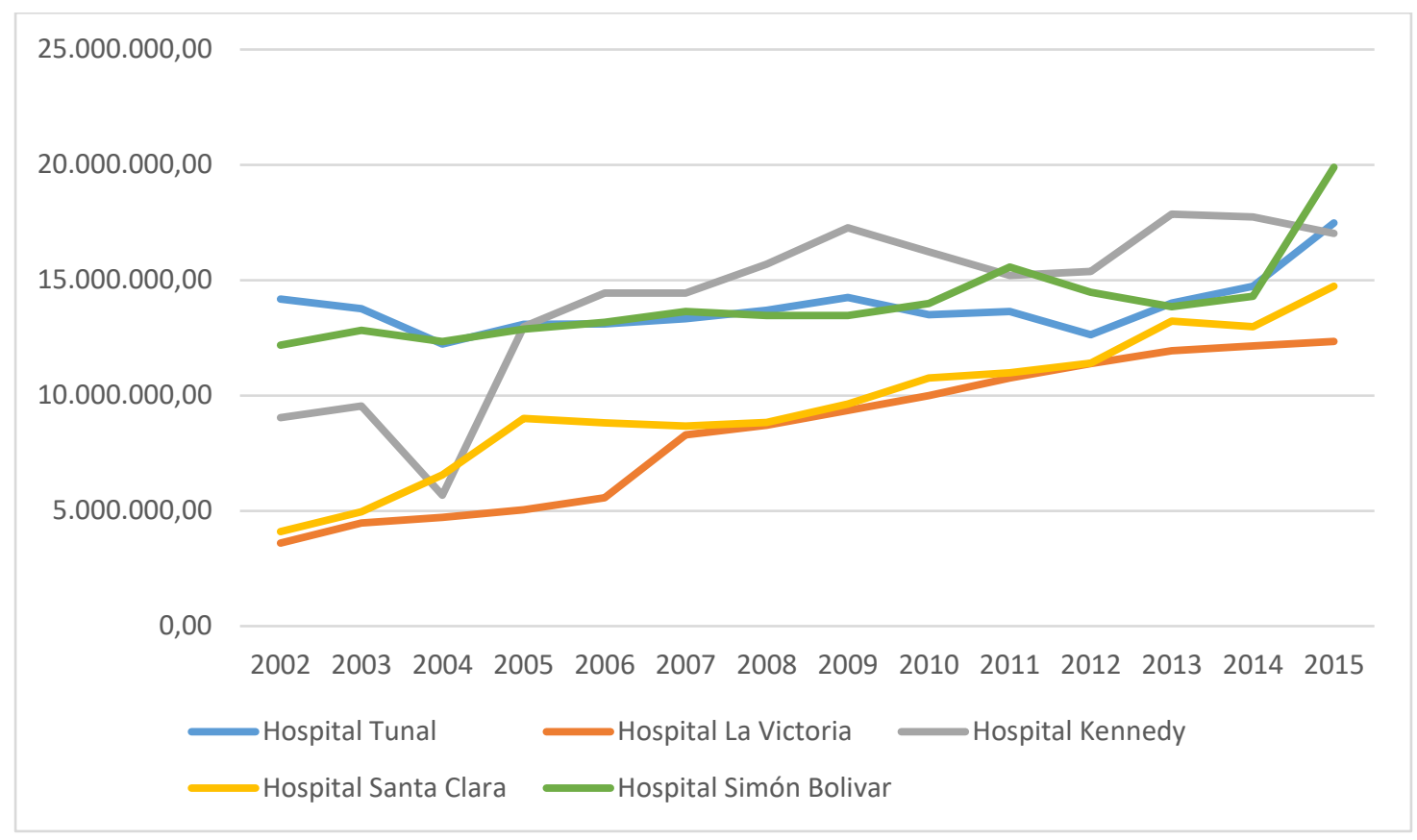

Fuente: Información tomada de la ficha técnica del Ministerio de Salud y Protección Social

La producción en UVR se encarga de establecer el uso de los recursos frente a los procedimientos médicos, es decir que mide cuanto uso se hace de instalaciones físicas dotadas para procedimientos especiales, la cantidad de medicamentos y material médico y el talento humano que se debe desplegar para atender un paciente, con este mecanismo valora la diferencia el entre un biológico aplicado (aplicar una vacuna) y atender un parto por cesárea. Con estas equivalencias se mide el comportamiento del gasto, el comportamiento de los ingresos y sirve para realizar comparaciones entre unidades hospitalarias. En el caso de los hospitales de III nivel de atención resulta claro, a juzgar por la figura anterior que todos los hospitales han atendido un crecimiento en la demanda de servicios, a excepción del hospital de Kennedy.

El Hospital de Kennedy es referente de atención para una de las zonas más convulsionadas del distrito capital, sin embargo, la principal puerta de entrada para la demanda espontánea de servicios es el servicio de urgencias y este ha estado en remodelación durante las vigencias sobre las cuales se aprecia precisamente una 
disminución de servicios de salud, que fueron los años 2013, 2014 y 2015. La producción revisada a partir del comportamiento de las unidades de valor relativo son una aproximación al comportamiento

La red pública hospitalaria ha tenido la siguiente evolución frente a la oferta de camas:

En el I Nivel la oferta de camas se ha mantenido durante el periodo 2002-2015, esto se debe a la baja ocupación que históricamente se ha presentado en los primeros niveles como se presenta en las siguientes figuras.

Figura 30 Camas de hospitalización I Nivel

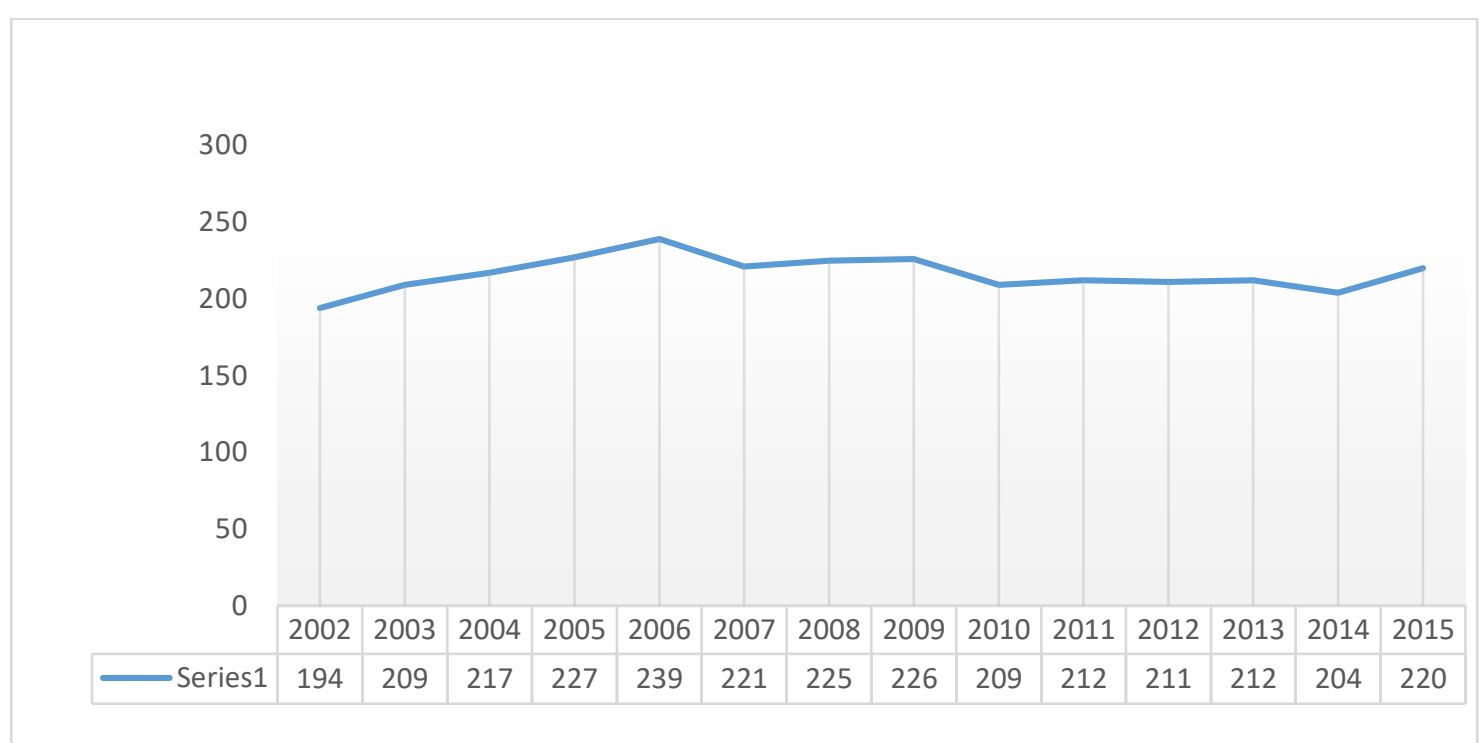

Fuente: Información tomada de la ficha técnica del Ministerio de Salud y Protección Social

La explicación parcial de la estabilidad de la oferta en las camas de hospitalización del primer nivel se fundamenta en el porcentaje de ocupación que se presenta en la siguiente figura. 


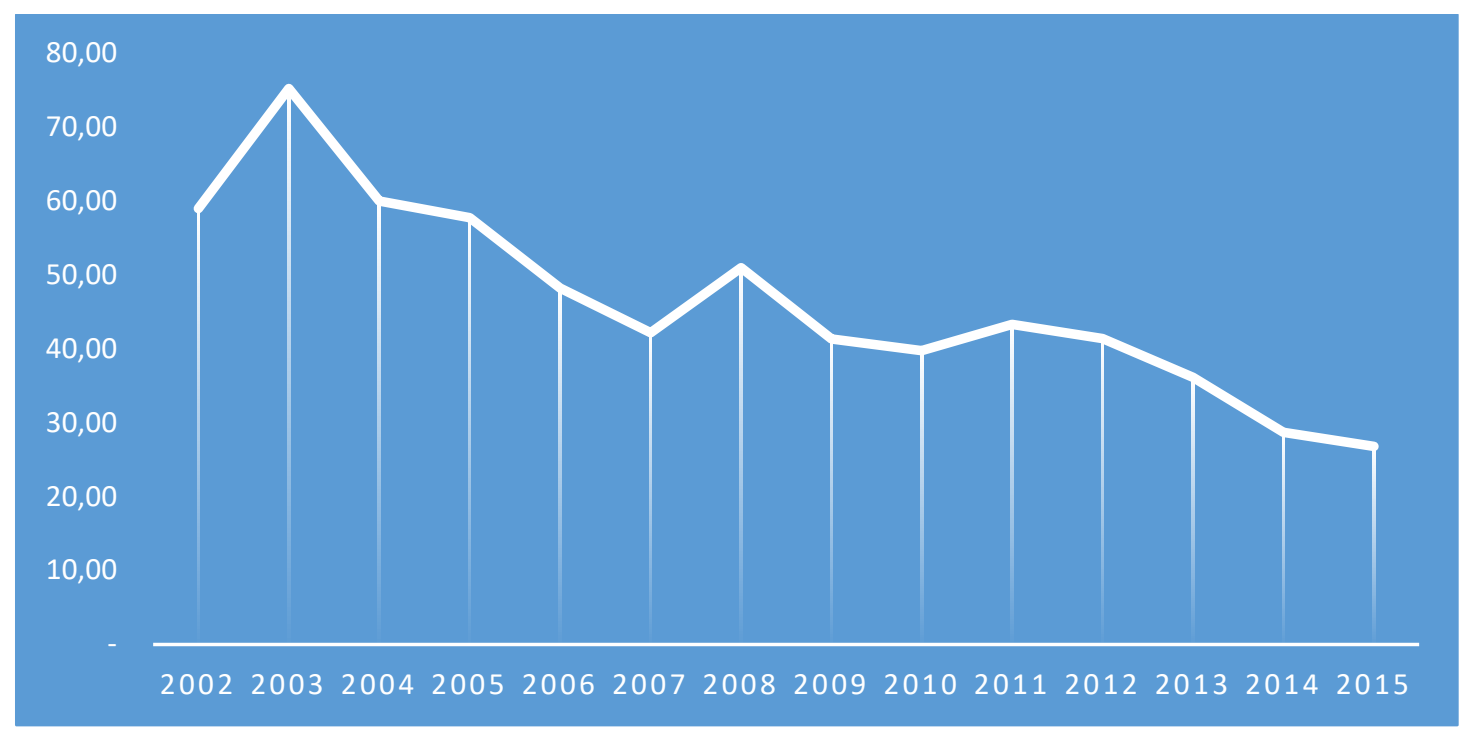

Fuente: Elaborado por el autor con base en información tomada de la ficha técnica del Ministerio de Salud y Protección Social

Estos promedios de ocupación son diferentes para las unidades hospitalarias como se presentará más adelante, sin embargo, el consolidado presenta un desinterés de la demanda por los servicios prestados en el primer nivel como se presenta en la siguiente figura.

Figura 32 Porcentaje ocupacional por unidad hospitalaria

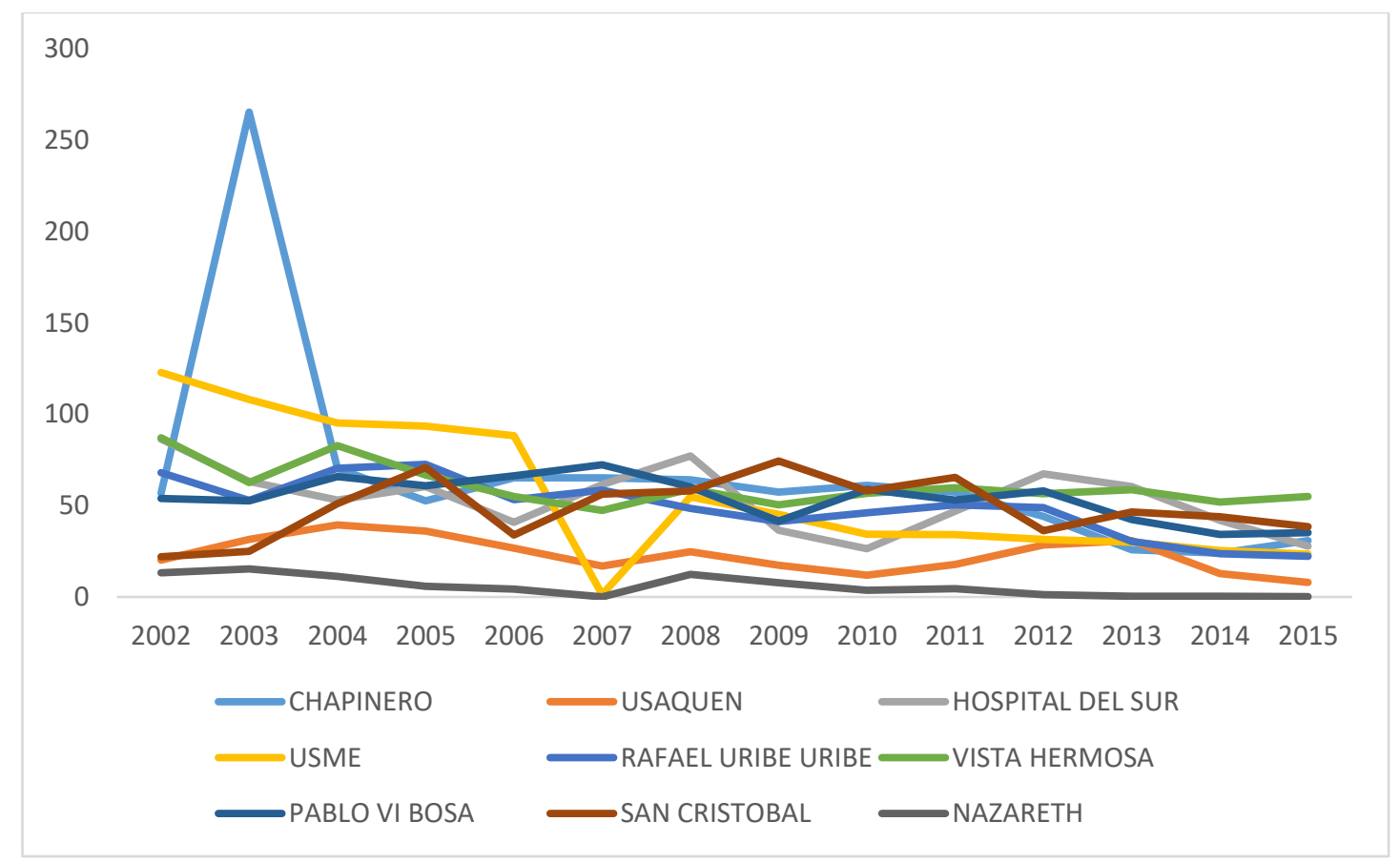

Fuente: Elaborado por el autor con base en información tomada de la ficha técnica del Ministerio de Salud y Protección Social 
La baja ocupación ha estancado las posibilidades de la oferta que crece con base en la demanda de servicios y no con base en la explosión demográfica, lo que implica que el número de camas por habitante disminuya, por lo menos para el primer nivel durante los años descritos.

En el II Nivel

Figura 33 Camas de hospitalización II Nivel de atención

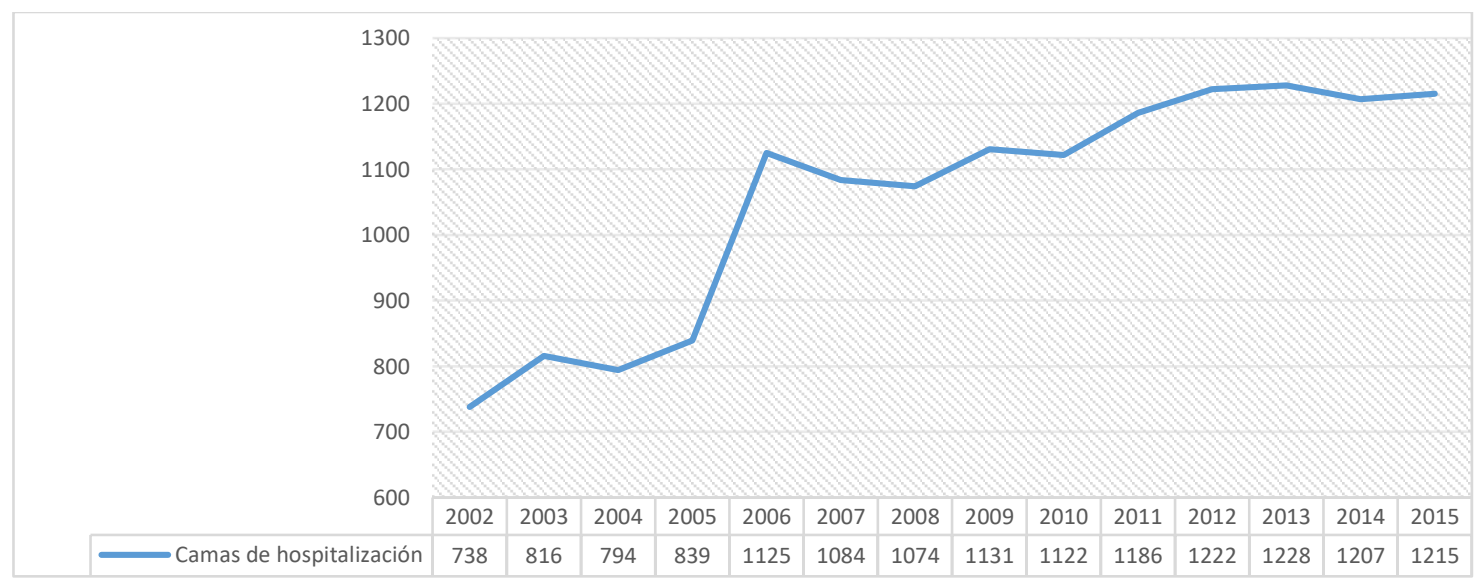

Fuente: Elaborado por el autor con base en información tomada de la ficha técnica del Ministerio de Salud y Protección Social

Las camas de hospitalización de la red pública de II Nivel de atención han evolucionado a una tasa media de $3,63 \%$ lo cual ha permitido controlar la media del porcentaje ocupacional en un $85 \%$. Este porcentaje de ocupación media es muy alto si se considera la estacionalidad y características de la oferta de servicios, la siguiente figura muestra la ocupación media del segundo nivel.

\section{Figura 34 Porcentaje Ocupacional II Nivel}

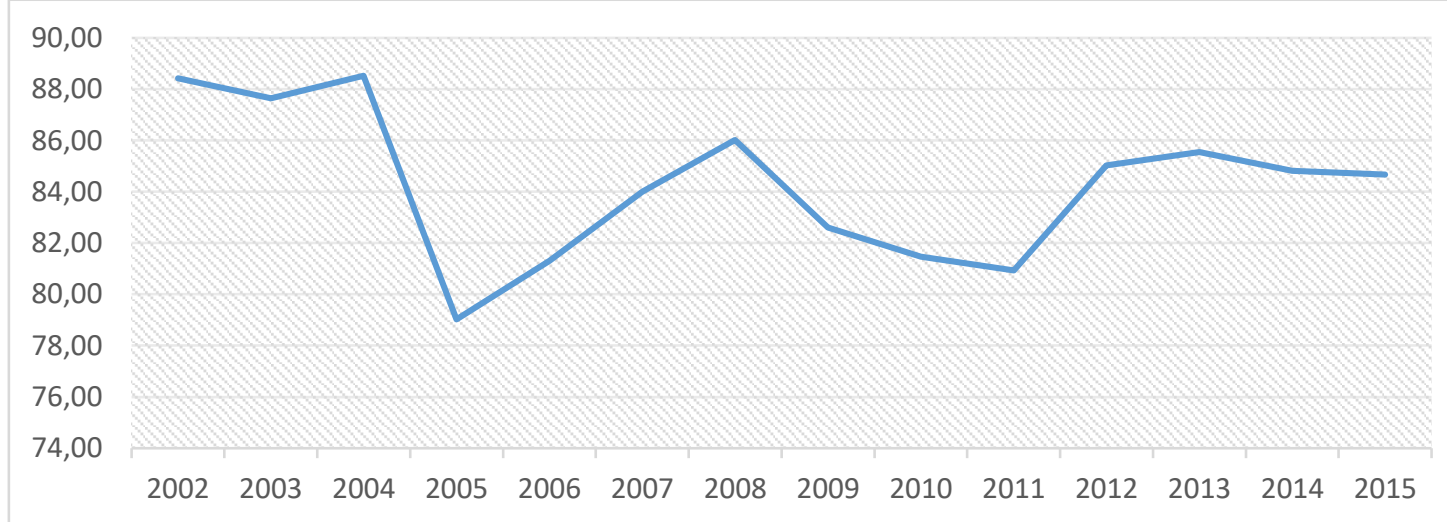

Fuente: Elaborado por el autor con base en información tomada de la ficha técnica del Ministerio de Salud y Protección Social 
Por otra parte, el total de egresos del segundo nivel ha presentado un comportamiento irregular, que termina en una cifra bastante similar a la del año de inicio del presente documento como se presenta en la siguiente figura.

Figura 35 Total egresos II Nivel

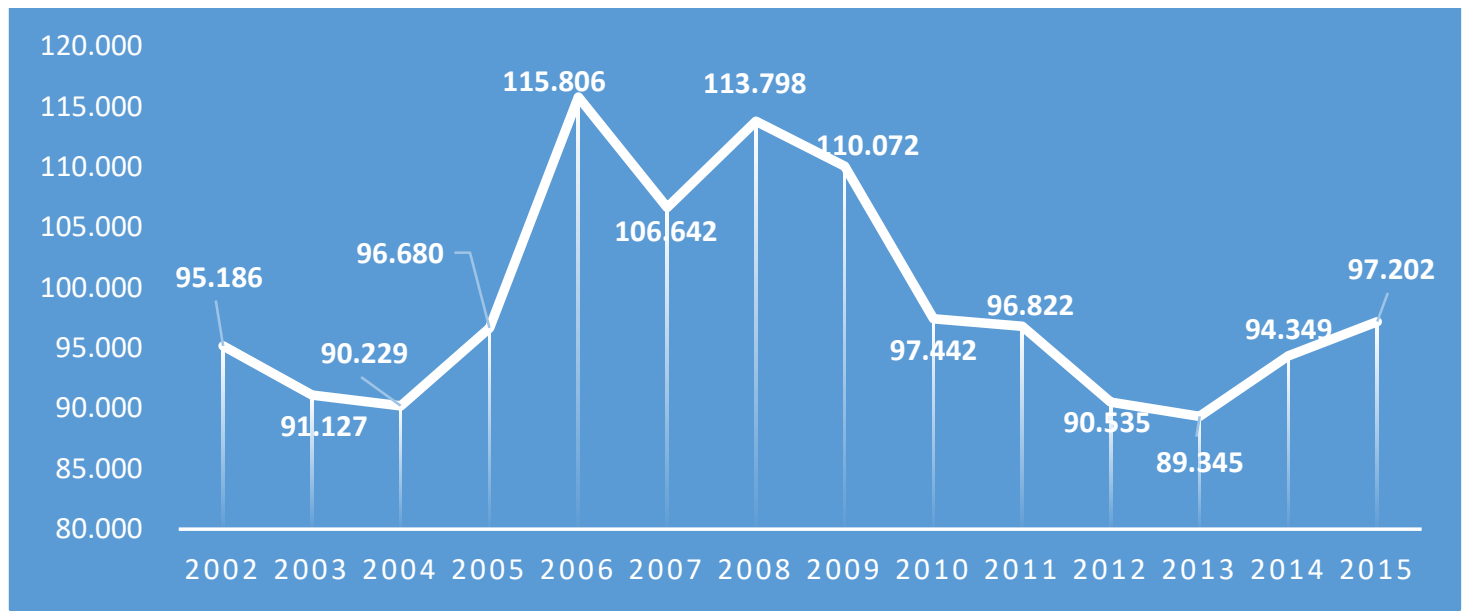

Fuente: Elaborado por el autor con base en información tomada de la ficha técnica del Ministerio de Salud y Protección Social

En el III Nivel la evolución de las camas y la ocupación ha sido la siguiente Figura 36 Camas de Hospitalización III nivel

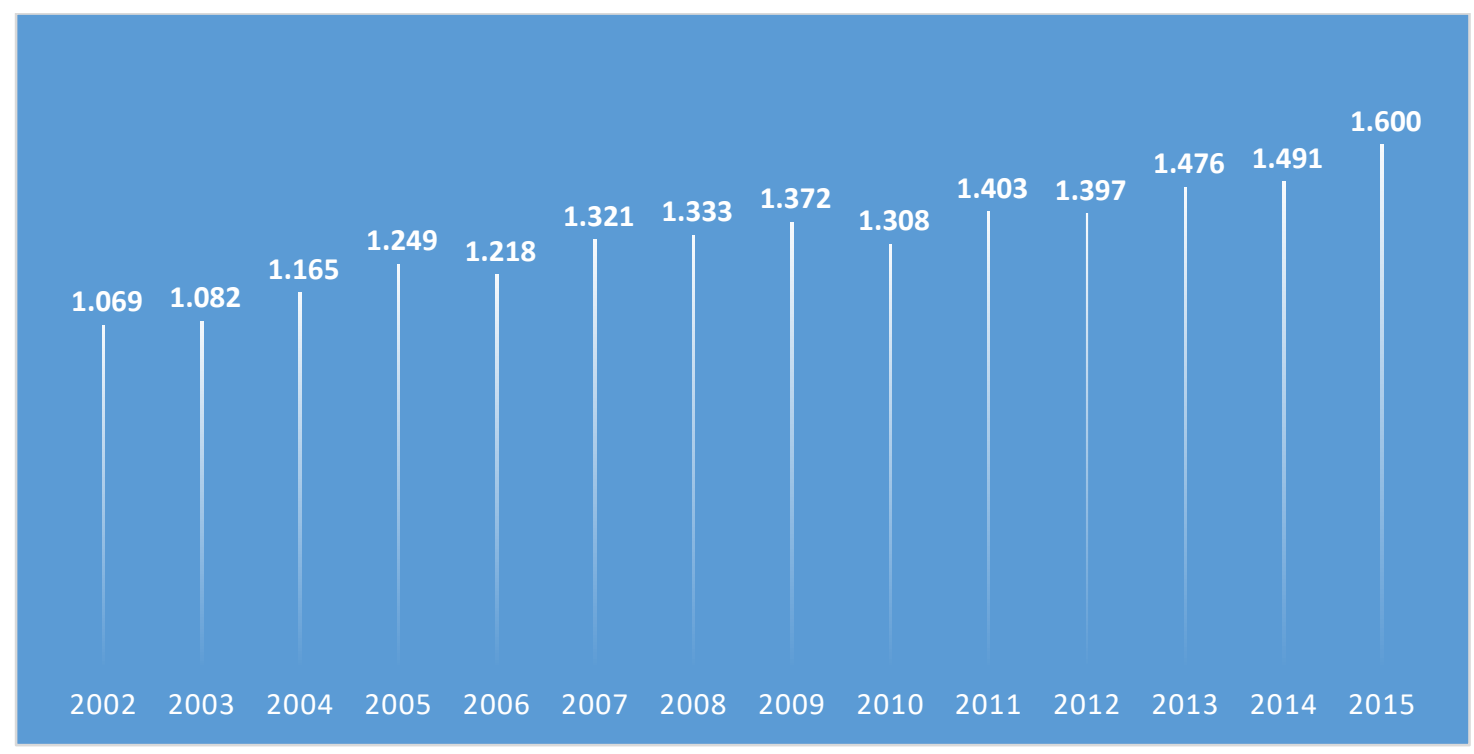

Fuente: Elaborado por el autor con base en información tomada de la ficha técnica del Ministerio de Salud y Protección Social

La tasa de incremento medio de las camas de hospitalización del tercer nivel ha sido cercana al 3\% anual mientras que los egresos se han comportado según la siguiente figura 
Figura 37 Total de camas por nivel de atención

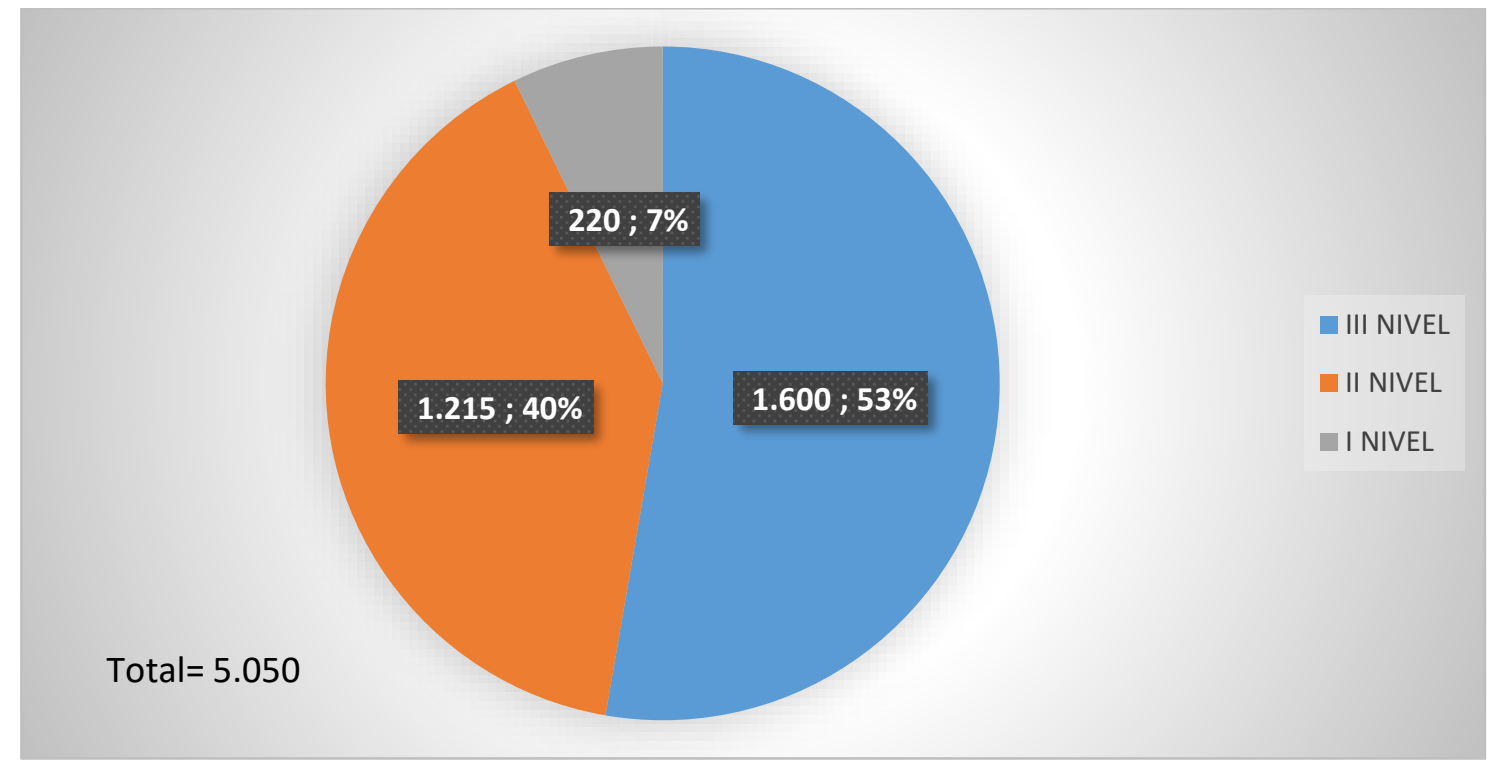

Fuente: Elaborado por el autor con base en información tomada de la ficha técnica del Ministerio de Salud y Protección Social

Figura 38 Total Egresos III Nivel

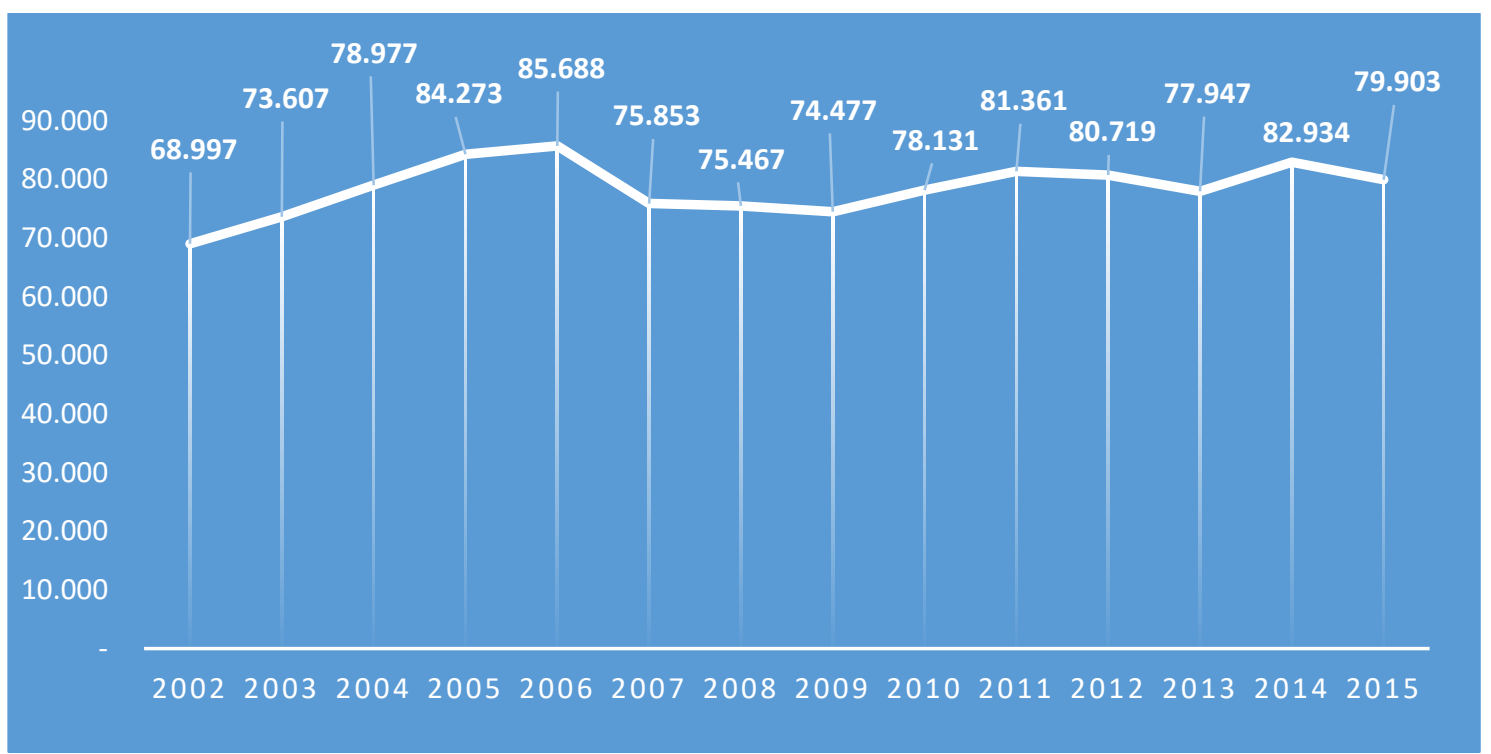

Fuente: Elaborado por el autor con base en información tomada de la ficha técnica del Ministerio de Salud y Protección Social

La ocupación media del III Nivel para 2015 es del 107\%, esta situación devela que la necesidad se concentra en la medicina especializada, en virtud de la ocupación consolidada de los diferentes niveles que muestra una baja ocupación para el I Nivel, una ocupación del $85 \%$ para el II Nivel y una superior al 100\% en el III Nivel. 


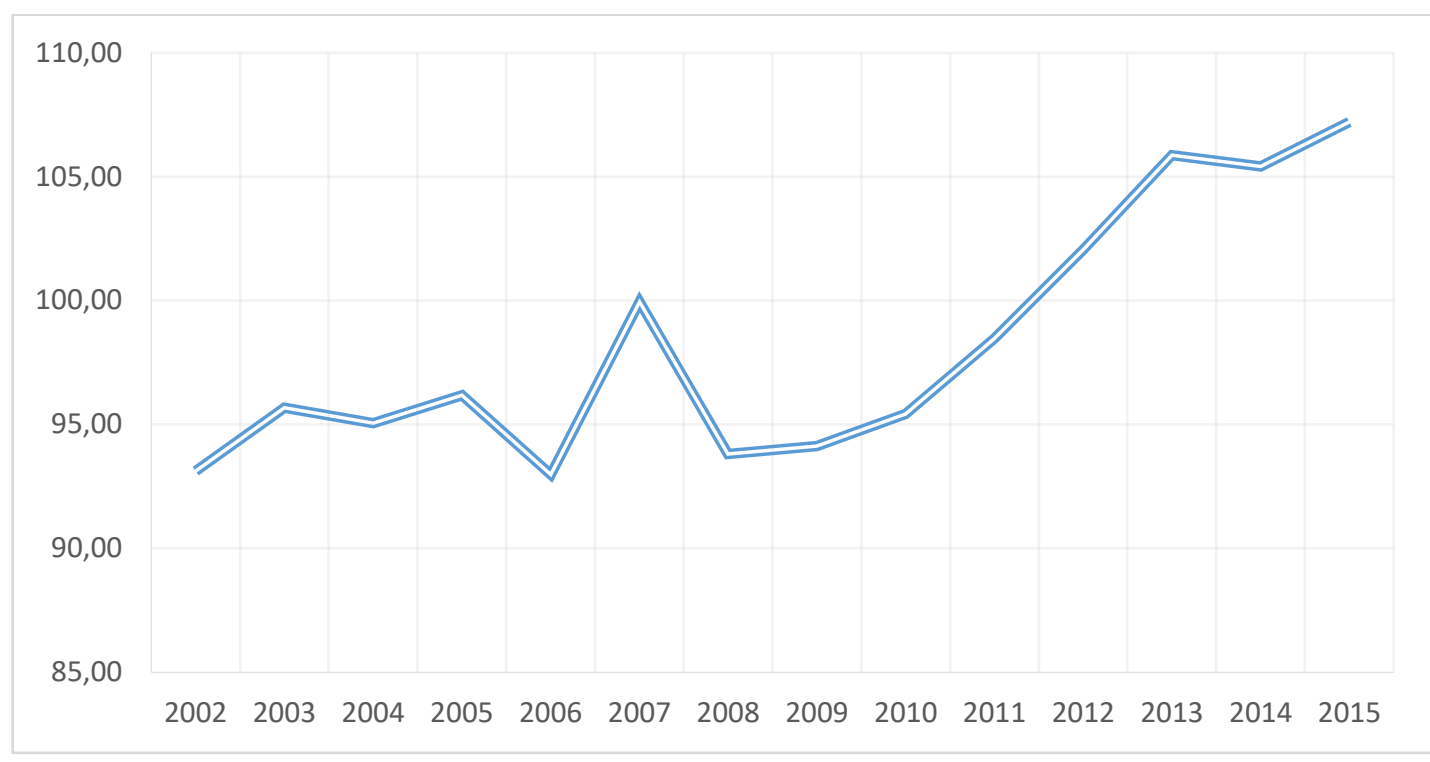

Fuente: Elaborado por el autor con base en información tomada de la ficha técnica del Ministerio de Salud y Protección Social

La presión de la demanda de servicios de alta complejidad y la escasa capacidad de respuesta por el porcentaje medio de ocupación del III nivel muestran que existe un déficit importante en el III nivel.

Las dificultades más apremiantes del sistema general de seguridad social en salud se concentran en el III Nivel, crecimiento de la cartera superior y desequilibrado con respecto al crecimiento de los pasivos que señalan una necesidad de cambio estructural del comportamiento de los "Jugadores" del sistema.

La propuesta es mitigar el riesgo debido a que la cámara de compensación se comporta como pagador ante el facturador y como facturador ante el pagador generando una estabilidad de las operaciones que logra a partir de una recuperación de fondos eficiente y de la exigencia a los operadores de depósitos de fondos adicionales en sus cuentas de corretaje con el fin de mantener un mínimo de requisitos de margen que estabiliza la integridad financiera del sistema.

En caso de que una de las partes incumpla la tarea que debe cumplir en el sistema, se debe implementar un mecanismo de suspensión de sus operaciones y/o sanciones con el fin de que no se asuma un riesgo excesivo en el sistema y uso automático de los recursos constitutivos de las garantías en las obligaciones contraídas. 


\title{
CAPÍTULO 2. EVALUACIÓN DE EFICIENCIA DE LA RED PÚBLICA HOSPITALARIA DE BOGOTÁ
}

\author{
"En general, la evaluación social no solo es \\ importante sino necesaria para que un país \\ logre optimizar el uso de los escasos \\ recursos con que cuenta." Nicholas Kaldor
}

La técnica del análisis envolvente de datos [DEA], mediante el modelo Banker, Charnes \& Cooper Output con un enfoque orientado a resultados [BCC-O] es la técnica implementada para el presente trabajo, esta técnica permite evaluar la eficiencia y realizar el análisis pertinente para cada una de las 22 IPS de la red pública hospitalaria del Distrito Capital en el periodo 2002-2015, la cual se va a adelantar por Niveles de Atención y para lo cual se ha tenido en cuenta información financiera e información científica del Sistema de Información Hospitalaria - SIHO del Ministerio de Salud y Protección Social.

Esto significa que los resultados se derivan de la siguiente ecuación

$$
\text { Eficiencia Administrativa }=\frac{\text { Eficiencia Financiera }+ \text { Eficiencia hospitalaria }}{2}
$$

Se promedia con el fin de seguir estableciendo los términos de optimización limitados en el intervalo 0 - 1

\subsection{Metodología}

Como instrumento metodológico se presenta la aplicación de análisis envolvente de datos DEA, este instrumento fue desarrollado en 1978 por Charnes, Cooper \& Rhodes, permite medir la eficiencia relativa de las Unidades de Toma de Decisión (Decision Making Unit - [DMU] por sus siglas en Inglés) en el que resulta posible utilizar múltiples entradas (Inputs o recursos) y salidas (outputs ó productos) con el fin de estimar una medida que se traduce en estimaciones de eficiencia e identificar cuales DMU son más eficientes frente a la información proporcionada; bien sea con enfoque en las entradas o evaluados a partir de las salidas; en los trabajos desarrollados por Farrel, Aigner y Chu y Afriat, Charnes, Cooper \& Rhodes complementaron esta técnica de análisis DEA y propusieron un proceso de linealización con el cual cada unidad evaluada mostraría sus pesos más favorables frente a las demás unidades evaluadas y se encontrarían las DMU 
acordes a la frontera de eficiencia, estableciendo de esta manera los índices de eficiencia más altos o bajos de las unidades objeto de estudio.

Las ventajas más reconocidas para utilizar esta herramienta de toma de decisiones son:

a) al no ser una técnica paramétrica, permite dejar las variables sin ningún tipo de ponderaciones. Es decir, DEA procede a dar estas ponderaciones en forma empírica y las determina para cada variable.

b) permite medir de manera simultánea unidades productivas o variables para la evaluación de eficiencia

c) optimiza la medida de eficiencia de cada variable analizada, para crear así una frontera eficiente identificando y cuantificando las DMU que resultan eficientes e ineficientes con relación a los resultados utilizados (inputs) y los resultados obtenidos (outputs) seleccionados.

El análisis envolvente de datos es uno de los más utilizados en la actualidad, tanto en el sector público como en el privado, para estimar el rendimiento de unidades de producción y realizar análisis de eficiencia que permitan realizan comparaciones, evaluaciones de desempeño en diferentes ámbitos económicos (salud, educación, financiero, entre otros).

A continuación, se presentan las variables más reconocidas de esta técnica no paramétrica.

\section{Modelo Banker, Charner y Cooper. Output orientado (BCC-O)}

En cuanto al modelo BCC-O nombrado así por sus desarrolladores Banker, Charnes \& Cooper -, es una extensión del primer modelo DEA CCR, su formulación es similar a este último, sin embargo, su diferencia radica en que BCC supone rendimientos variables a escala, es decir, los incrementos en los productos van a estar caracterizados de la siguiente manera:

- Rendimientos constantes a escala: Un aumento en los productos (salidas) de la DMU son consecuencia de un aumento proporcional en los insumos (entradas) de la misma DMU. 
- Rendimientos crecientes a escala: El aumento porcentual de las salidas de la DMU será mayor al aumento porcentual de los factores (entradas) en la misma DMU.

- Rendimientos decrecientes a escala: El incremento porcentual de los Outputs de la DMU evaluada será menor al incremento porcentual de los Inputs en dicha DMU.

Una DMU se considera $100 \%$ eficiente cuando ninguna de sus entradas puede ser disminuido sin reducir algunas de sus salidas o aumentar algunas de sus entradas y, ninguna de sus salidas puede aumentar sin incrementar cualquiera de sus entradas o reducir una o más de sus salidas; en este sentido la eficiencia está definida como una relación ponderada entre los Outputs y los Inputs utilizados en el conjunto de elementos (Charnes, Cooper , \& Rhodes, November 1978).

Una de las maneras por las que se clasifican los modelos DEA es la orientación del modelo, dicha orientación se puede enfocar hacia sus Inputs o hacia sus Output. En el caso de la presente investigación el enfoque está orientado a las salidas (BCC-O), debido principalmente a que las IPS no se hallan expuestas a un mercado competitivo sino a un mercado con un alto componente de regulación y en consecuencia resulta conveniente que tanto pueden lograr con inputs equivalentes.

La forma funcional del modelo BCC-O para el presente desarrollo es la siguiente: es la siguiente:

$$
\text { Modelo } B C C-O=\frac{\left\{\begin{array}{c}
\text { Min } Z=V x_{j}+k_{0} \\
\text { Sujeto } a: \\
U y_{j}=1 \quad j=1,2, \ldots, 9 \\
V X+k_{0} \geq U Y \\
V Z \geq 0 \\
k_{0} \text { No restringida }
\end{array}\right\}+\left\{\begin{array}{c}
\text { Min } Z=V x_{j}+k_{0} \\
\text { Sujeto } a: \\
U y_{j}=1 \quad j=1,2, \ldots, 9 \\
V X+k_{0} \geq U Y \\
V Z \geq 0 \\
k_{0} \text { No restringida }
\end{array}\right\}}{2}
$$

Donde:

$\mathrm{Z}=$ Índice de eficiencia en la administración de los recursos

$\mathrm{X}=$ Vector de inputs

$\mathrm{X}_{\mathrm{j}}=$ vector de inputs de la unidad evaluada

$\mathrm{Y}=$ Vector de Outputs

$y_{j}=$ Vector de Ouputs de la unidad evaluada

$\mathrm{UV}=$ Vectores fila de outputs e inputs respectivamente 
$\mathrm{K}_{0}=$ Término constante

La ecuación representa el problema lineal para el conjunto de DMU objeto de estudio, el cual busca maximizar el Output dado el nivel de Inputs, sujeto: 1) a la eficiencia del conjunto de elementos, 2) a que las variables del problema ( $\mathrm{U}$ y V) sean mayores o iguales a cero con el fin de evitar que alguna de las entradas o la salida sea ignorada en la determinación de la eficiencia, y por último, 3) al valor que tome la constante, la cual determinara el tipo de rendimientos a escala para la unidad evaluada.

\subsection{Medida de Eficiencia}

El DEA es una técnica no paramétrica de medición de eficiencia que se basa en la estimación de una frontera de eficiencia derivada de un conjunto de observaciones sin acudir a una función de producción, por lo tanto, no se requiere una relación funcional entre los elementos de entrada y las salidas; esto convierte el modelo en una alternativa para extraer información de diversos objetos de estudio y por lo tanto más versátil. El modelo construye una frontera de producción empírica y después evalúa la eficiencia de cada unidad observada que no haya alcanzado a la frontera de eficiencia (Post \& Spronk, 1999).

El DEA se considera no paramétrico porque no requiere de una función de relación entre las entradas y las salidas; no es estadístico porque no asume que la eficiencia siga algún tipo de distribución probabilística (Peña, Marroquín, \& Ríos, 2008).

En DEA la eficiencia vendrá medida en una escala de 0 a 1 , donde un valor de indicar que la unidad evaluada es eficiente respecto a las demás. Por el contrario, si, indicara que la DMU está siendo ineficiente en la administración de sus recursos.

En cuanto al término constante de la ecuación antes representada, si:

\begin{tabular}{|l|}
\hline $\mathrm{K}_{\mathrm{o}}<0$; predominan los rendimientos decrecientes a escala \\
\hline $\mathrm{K}_{\mathrm{o}}=1$; predominan los rendimientos constantes a escala \\
\hline $\mathrm{K}_{\mathrm{o}}>1$; predominan los rendimientos crecientes a estala \\
\hline
\end{tabular}




\subsection{Criterios de Inclusión, exclusión y clasificación de resultados}

Las siguientes son las características para que una unidad de decisión (DMU) sea parte de la muestra de estudio.

Cuando se adelanta un análisis de eficiencia se debe entender que los modelos DEA nos dan una guía para tomar decisiones de mejora, en pos optimizar la utilización de los recursos, en términos matemáticos, por lo tanto se considera que variables (entrada y salida) deberán estar ubicadas en el primer cuadrante del plano cartesiano para poder ser analizadas dentro de una frontera de posibilidades; lo anterior significará que las IPS que presenten información negativa en las variables seleccionadas no podrán ser tenidas en cuenta.

Adicionalmente se propone analizar dos perspectivas de resultados; en la perspectiva financiera se propone categorizar como mejores unidades de negocio a las que presenten rendimientos decrecientes a escala, con lo que se busca que, ante variaciones de los ingresos corrientes y cuentas por cobrar, exista una disminución de los gastos totales y las cuentas por pagar, con el fin de buscar la estabilidad financiera y cumplimiento del objetivo básico financiero.

Desde la perspectiva hospitalaria se ha definido establecer como las mejores unidades de negocio a aquellas que logren rendimientos crecientes, es decir que, definida la cantidad del gasto, se presente una variación positiva de la producción.

Adicionalmente se informa que los recursos que recibieron las DMU por cuenta del cumplimiento de los programas de saneamiento fiscal y financiero

Desde la perspectiva financiera, las entradas, Recaudo corriente y recaudo de cuentas por cobrar, se escogieron estas dos variables por cuanto que la operación de las Empresas Sociales del Estado se define a partir del comportamiento de la caja, es decir que el presupuesto subordina la operación financiera como se explicó anteriormente. El origen principal de los recursos que apalancan la operación son el ingreso corriente el cual se afecta por el proceso de facturación, radicación, reconocimiento y pago, que puede significar un rezago de 2 meses, es decir que la facturación de enero puede estar ingresando en marzo o abril, esto obliga a que los dos o tres primeros meses de operación se apalancan con la recuperación de cuentas por cobrar. 
Existen otros ingresos que contribuyen en menor porcentaje como la facturación de la población pobre no asegurada, la facturación al régimen contributivo, otras ventas de servicio, aportes y otros ingresos (por ejemplo, por venta de activos inservibles)

Las variables de salida escogidas son gasto total comprometido y cuentas por pagar, estos dos rubros reúnen el $100 \%$ del uso de los recursos de efectivo.

Desde la perspectiva de Gestión Hospitalaria se escogieron el valor total de los compromisos (gasto total comprometido más cuentas por pagar con el fin de enlazar con la perspectiva financiera) y la producción total en UVR, con esta relación se busca que definidos los recursos financieros en materia de producción se use de la mejor forma.

El modelo desarrollado se considera endógeno, motivo por el cual se advierten distorsiones en el 2015, derivadas de los recursos de cuentas maestras que palearon la situación financiera de las DMU que adoptaron programas de saneamiento fiscal y financiero.

\subsection{Resultados}

Los resultados generales muestran que el benchmarking o DMU que cumple con suficiencia los supuestos de rendimientos decrecientes para la perspectiva financiera y rendimientos crecientes para la perspectiva de Gestión Hospitalaria cambian durante los años observados como se presenta a continuación.

\subsubsection{Resultados I Nivel}

En el primer nivel desde la perspectiva financiera 7 de 9 DMU presentan rendimientos marginales decrecientes para el año 2015, lo cual es coherente con las hipótesis de respuesta del modelo, un hospital presenta rendimientos constantes a escala, es decir que la proporción del gasto comprometido y cuentas por cobrar crecen al mismo ritmo que los ingresos corrientes y las cuentas por cobrar, esta DMU es Rafael Uribe Uribe y con rendimientos crecientes a escala el Hospital San Cristobal

Esto significa que los hospitales de primer nivel no presentan grandes dificultades financieras, esto derivado del tamaño de las operaciones y del porcentaje de ocupación como se presentó en el capítulo 2.

Desde la perspectiva de Gestión Hospitalaria hay una DMU que se comporta de forma consistente como el benchmark del primer nivel es el Hospital Pablo VI Bosa, es el 
referente del primer nivel por cuanto desde la perspectiva financiera logra rendimientos marginales decrecientes con un porcentaje que se aproxima al 1 como se puede apreciar en el Anexo

Los resultados son los siguientes

Tabla 14 Eficiencia financiera I Nivel

\begin{tabular}{|l|c|c|c|c|c|c|c|c|c|c|c|c|c|c|}
\hline AÑOS & $\mathbf{2 0 0 2}$ & $\mathbf{2 0 0 3}$ & $\mathbf{2 0 0 4}$ & $\mathbf{2 0 0 5}$ & $\mathbf{2 0 0 6}$ & $\mathbf{2 0 0 7}$ & $\mathbf{2 0 0 8}$ & $\mathbf{2 0 0 9}$ & $\mathbf{2 0 1 0}$ & $\mathbf{2 0 1 1}$ & $\mathbf{2 0 1 2}$ & $\mathbf{2 0 1 3}$ & $\mathbf{2 0 1 4}$ & $\mathbf{2 0 1 5}$ \\
\hline Chapinero & 0,90 & 0,84 & 0,82 & 1,00 & 0,69 & 0,82 & 0,66 & 0,71 & 0,63 & 0,84 & 0,65 & 0,67 & 1,00 & 0,97 \\
\hline Del Sur & 0,77 & 0,75 & 0,65 & 0,68 & 0,82 & 0,66 & 0,68 & 0,69 & 0,84 & 0,68 & 0,54 & 0,65 & 0,74 & 0,80 \\
\hline Nazareth & 0,90 & 0,90 & 0,95 & 0,99 & 0,97 & 1,00 & 0,98 & 0,98 & 0,99 & 1,00 & 1,00 & 0,94 & 1,00 & 0,97 \\
\hline Pablo VI Bosa & 1,00 & 0,92 & 1,00 & 0,82 & 1,00 & 0,99 & 1,00 & 0,95 & 1,00 & 0,88 & 0,73 & 0,87 & 0,85 & 0,93 \\
\hline Rafael Uribe U & 0,87 & 0,84 & 0,74 & 0,71 & 0,73 & 0,74 & 0,74 & 0,73 & 0,67 & 0,63 & 0,70 & 0,64 & 0,64 & 1,00 \\
\hline San Cristóbal & 0,95 & 1,00 & 0,90 & 0,92 & 0,83 & 0,91 & 0,91 & 0,92 & 0,89 & 0,94 & 1,00 & 1,00 & 1,00 & 1,00 \\
\hline Usaquén & 0,87 & 0,84 & 0,76 & 0,89 & 0,97 & 0,83 & 0,91 & 0,95 & 0,91 & 0,87 & 0,96 & 0,87 & 1,00 & 0,97 \\
\hline Usme & 0,73 & 0,77 & 0,70 & 0,75 & 0,83 & 0,79 & 0,79 & 0,80 & 0,86 & 0,84 & 0,87 & 0,86 & 1,00 & 0,88 \\
\hline Vista Hermosa & 1,00 & 0,79 & 0,80 & 0,86 & 0,82 & 0,85 & 0,87 & 1,00 & 0,92 & 0,93 & 0,74 & 0,77 & 0,79 & 0,94 \\
\hline
\end{tabular}

Fuente: Elaborado por el autor con base en las salidas del modelo DEA desarrollado en Stata 2017

Dos de las unidades presentan resultados que se pueden señalar como ineficientes, el Hospital del Sur y el hospital de Usme, esto significa que no logran el equilibrio financiero y que en el modelo establecido no logran los objetivos de evaluación propuestos sobre disminución de cuentas por pagar.

El uno (1) se convierte en el objetivo eficiencia, por lo tanto, los valores inferiores desde la perspectiva financiera lo que implican es que se ha presentado una situación en la que las cuentas por cobrar crecen mas rápido que los ingresos, motivo por el cual, el referente de la frontera de posibilidades se mueve en torno al objetivo de eficiencia.

La distancia existente entre el índice de eficiencia para cada DMU y la unidad es la ineficiencia de cada DMU, es decir que los hospitales con indicadores mas alejados de la unidad, también son los mas ineficientes.

En la siguiente figura se aprecia de mejor manera la trazabilidad por DMU durante el periodo objeto de estudio frente a la evaluación de eficiencia financiera 


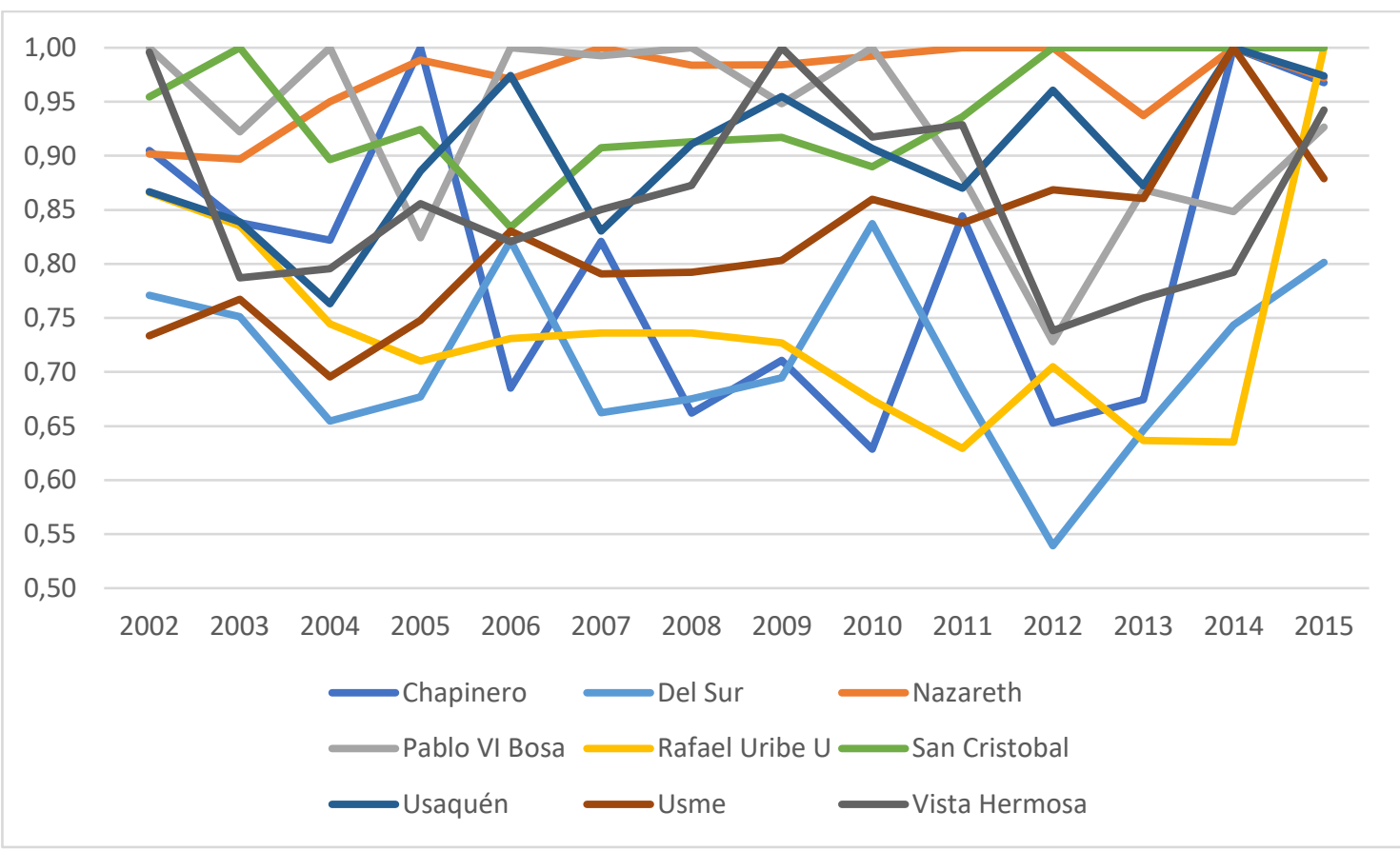

Fuente: Elaborado por el autor con base en las salidas del modelo DEA desarrollado en Stata 2017

Desde la perspectiva de Gestión Hospitalaria los resultados son los siguientes

Tabla 15 Eficiencia de gestión hospitalaria I Nivel

\begin{tabular}{|l|r|r|r|r|r|r|r|r|r|r|r|r|r|r|}
\hline AÑOS & $\mathbf{2 0 0 2}$ & $\mathbf{2 0 0 3}$ & $\mathbf{2 0 0 4}$ & $\mathbf{2 0 0 5}$ & $\mathbf{2 0 0 6}$ & $\mathbf{2 0 0 7}$ & $\mathbf{2 0 0 8}$ & $\mathbf{2 0 0 9}$ & $\mathbf{2 0 1 0}$ & $\mathbf{2 0 1 1}$ & $\mathbf{2 0 1 2}$ & $\mathbf{2 0 1 3}$ & $\mathbf{2 0 1 4}$ & $\mathbf{2 0 1 5}$ \\
\hline Chapinero & 0,94 & 0,94 & 0,81 & 0,89 & 0,90 & 0,95 & 0,95 & 0,95 & 0,96 & 0,96 & 0,95 & 0,96 & 0,95 & 0,96 \\
\hline Del Sur & 0,87 & 0,92 & 0,94 & 0,95 & 0,97 & 0,97 & 0,98 & 0,97 & 0,97 & 0,97 & 0,98 & 0,98 & 0,98 & 0,98 \\
\hline Nazareth & 0,97 & 0,98 & 0,99 & 0,99 & 1,00 & 1,00 & 1,00 & 0,97 & 0,92 & 0,93 & 0,96 & 0,85 & 0,82 & 0,78 \\
\hline Pablo VI Bosa & 1,00 & 1,00 & 1,00 & 1,00 & 1,00 & 1,00 & 1,00 & 1,00 & 1,00 & 1,00 & 1,00 & 1,00 & 1,00 & 1,00 \\
\hline Rafael Uribe U & 0,94 & 0,79 & 0,80 & 0,86 & 0,93 & 0,95 & 0,95 & 0,95 & 0,95 & 0,96 & 0,96 & 0,97 & 0,98 & 0,98 \\
\hline San Cristóbal & 0,98 & 0,99 & 0,98 & 0,99 & 0,99 & 1,00 & 1,00 & 0,96 & 0,81 & 0,79 & 0,70 & 0,51 & 0,46 & 0,44 \\
\hline Usaquén & 0,94 & 0,72 & 0,79 & 0,86 & 0,92 & 0,92 & 0,89 & 0,92 & 0,91 & 0,92 & 0,91 & 0,96 & 0,96 & 0,96 \\
\hline Usme & 0,92 & 0,95 & 0,96 & 0,97 & 0,98 & 0,98 & 0,98 & 0,99 & 0,99 & 0,99 & 0,98 & 0,99 & 1,00 & 1,00 \\
\hline Vista Hermosa & 0,97 & 0,97 & 0,96 & 0,98 & 0,98 & 0,99 & 0,99 & 0,99 & 1,00 & 0,99 & 0,99 & 0,99 & 0,99 & 0,99 \\
\hline
\end{tabular}

Fuente: Elaborado por el autor con base en las salidas del modelo DEA desarrollado en Stata 2017

Esto indica que en el I nivel solo un Hospital presenta información atípica, que es el Hospital San Cristóbal, esta DMU presenta en promedio una ocupación inferior al 50\%, tiene habilitadas 12 camas, y la producción por UVR se encuentra en el mismo nivel de 2012

En la siguiente figura se aprecia de mejor manera la trazabilidad por DMU durante el periodo objeto de estudio frente a la evaluación de eficiencia de gestión hospitalaria 


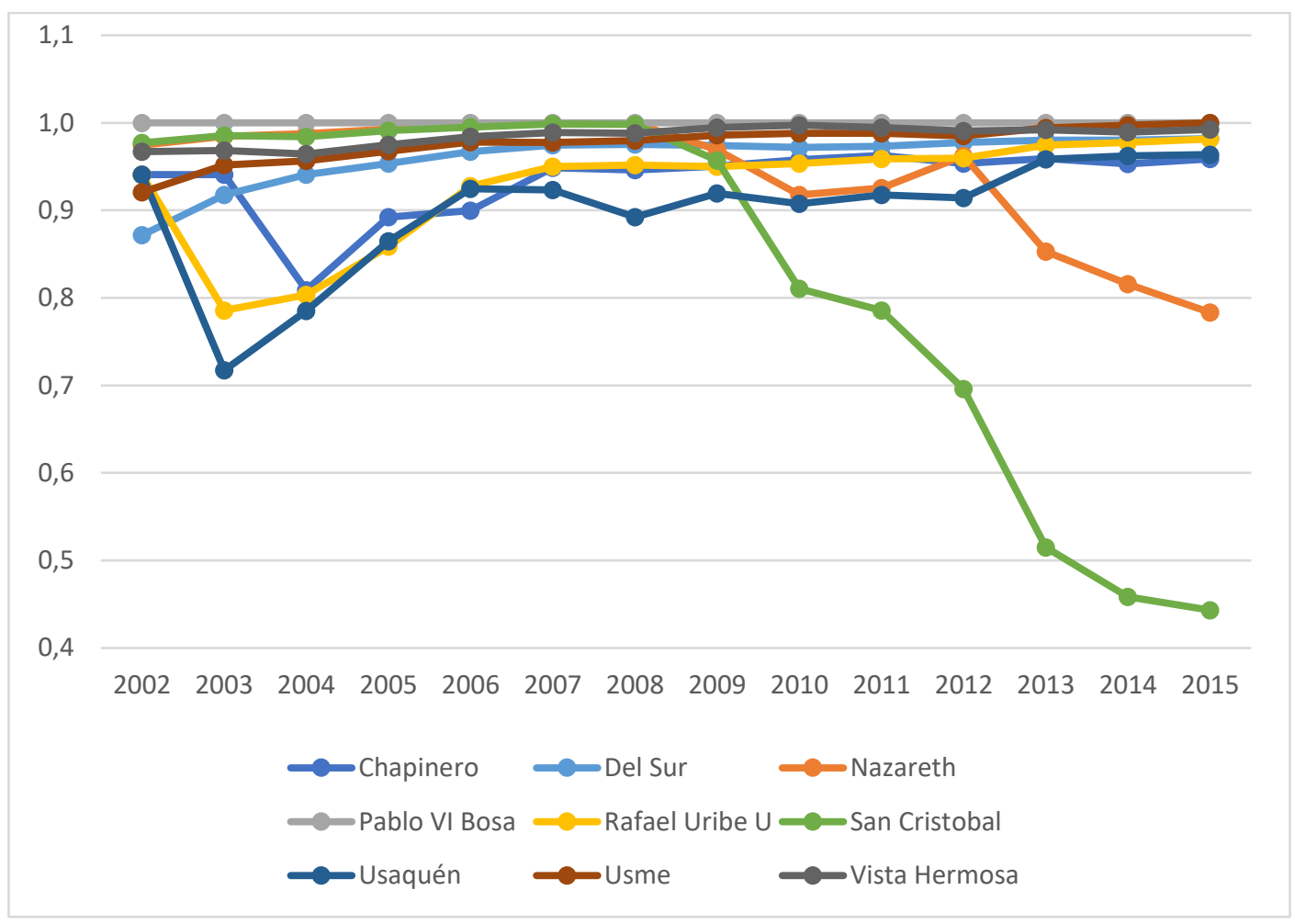

Fuente: Elaborado por el autor con base en las salidas del modelo DEA desarrollado en Stata 2017

Se puede concluir de forma preliminar que Nazareth y San Cristóbal quedan fuera de los estándares de comportamiento de los demás las demás unidades de I Nivel

El consolidado de acuerdo con la propuesta de evaluación los resultados son los siguientes Tabla 16 Eficiencia consolidados I Nivel

\begin{tabular}{|l|r|r|r|r|r|r|r|r|r|r|r|r|r|r|}
\hline AÑOS & $\mathbf{2 0 0 2}$ & $\mathbf{2 0 0 3}$ & $\mathbf{2 0 0 4}$ & $\mathbf{2 0 0 5}$ & $\mathbf{2 0 0 6}$ & $\mathbf{2 0 0 7}$ & $\mathbf{2 0 0 8}$ & $\mathbf{2 0 0 9}$ & $\mathbf{2 0 1 0}$ & $\mathbf{2 0 1 1}$ & $\mathbf{2 0 1 2}$ & $\mathbf{2 0 1 3}$ & $\mathbf{2 0 1 4}$ & $\mathbf{2 0 1 5}$ \\
\hline Chapinero & 0,92 & 0,89 & 0,82 & 0,95 & 0,79 & 0,88 & 0,80 & 0,83 & 0,79 & 0,90 & 0,80 & 0,82 & 0,98 & 0,96 \\
\hline Del Sur & 0,82 & 0,83 & 0,80 & 0,82 & 0,89 & 0,82 & 0,83 & 0,83 & 0,90 & 0,83 & 0,76 & 0,81 & 0,86 & 0,89 \\
\hline Nazareth & 0,94 & 0,94 & 0,97 & 0,99 & 0,98 & 1,00 & 0,99 & 0,98 & 0,95 & 0,96 & 0,98 & 0,90 & 0,91 & 0,88 \\
\hline Pablo VI Bosa & 1,00 & 0,96 & 1,00 & 0,91 & 1,00 & 1,00 & 1,00 & 0,97 & 1,00 & 0,94 & 0,86 & 0,93 & 0,92 & 0,96 \\
\hline Rafael Uribe U & 0,90 & 0,81 & 0,77 & 0,78 & 0,83 & 0,84 & 0,84 & 0,84 & 0,81 & 0,79 & 0,83 & 0,81 & 0,81 & 0,99 \\
\hline San Cristóbal & 0,97 & 0,99 & 0,94 & 0,96 & 0,91 & 0,95 & 0,96 & 0,94 & 0,85 & 0,86 & 0,85 & 0,76 & 0,73 & 0,72 \\
\hline Usaquén & 0,90 & 0,78 & 0,77 & 0,88 & 0,95 & 0,88 & 0,90 & 0,94 & 0,91 & 0,89 & 0,94 & 0,92 & 0,98 & 0,97 \\
\hline Usme & 0,83 & 0,86 & 0,83 & 0,86 & 0,90 & 0,88 & 0,89 & 0,89 & 0,92 & 0,91 & 0,93 & 0,93 & 1,00 & 0,94 \\
\hline Vista Hermosa & 0,98 & 0,88 & 0,88 & 0,92 & 0,90 & 0,92 & 0,93 & 1,00 & 0,96 & 0,96 & 0,86 & 0,88 & 0,89 & 0,97 \\
\hline
\end{tabular}

Fuente: Elaborado por el autor con base en las salidas del modelo DEA desarrollado en Stata 2017

En la siguiente figura se aprecia de mejor manera la trazabilidad por DMU durante el periodo objeto de estudio frente a la evaluación de eficiencia consolidada del I Nivel 


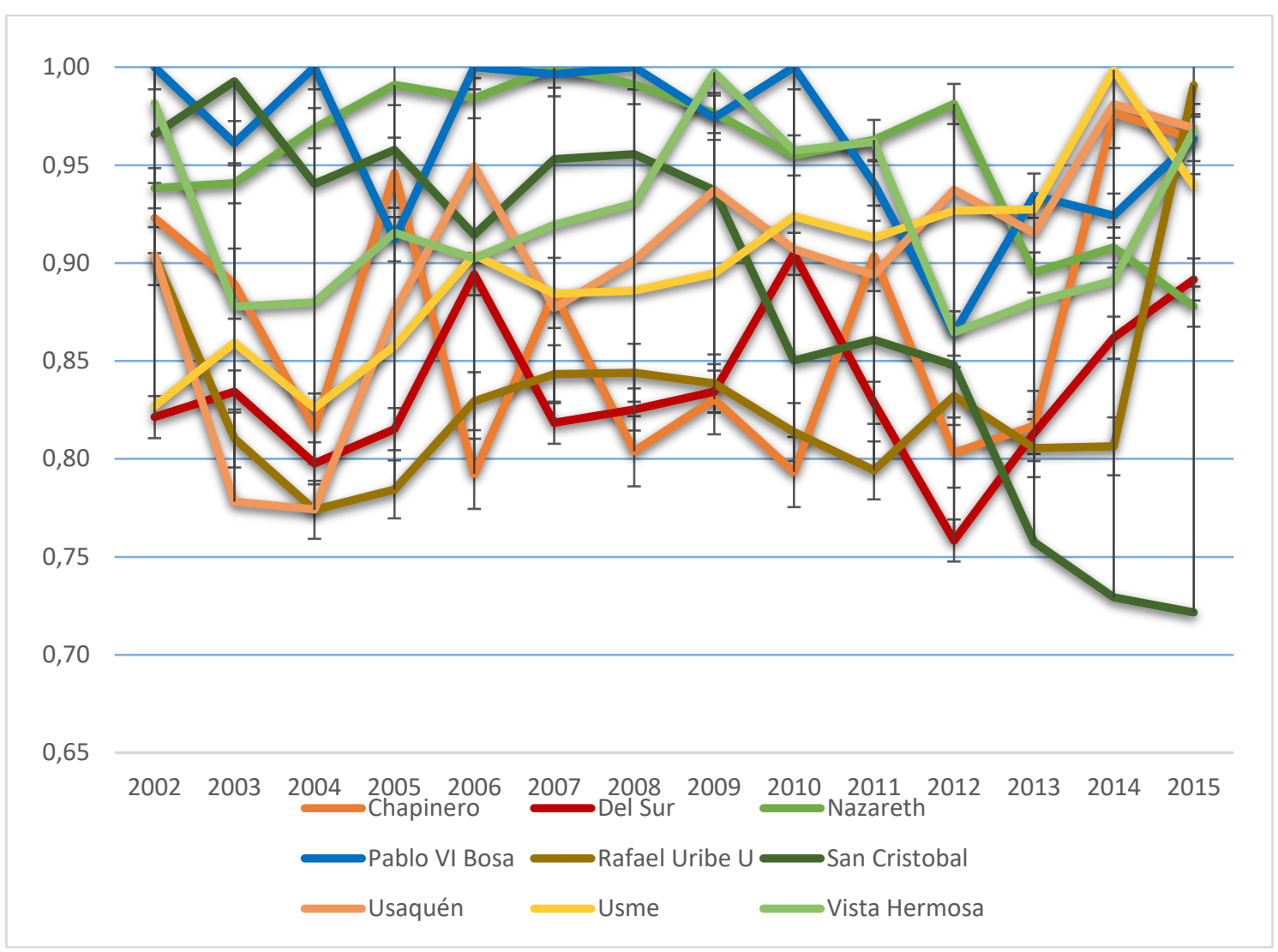

Fuente: Elaborado por el autor con base en las salidas del modelo DEA desarrollado en Stata 2017

\subsubsection{Resultados II Nivel}

En el II Nivel Suba, Engativá y Fontibón presentan rendimientos marginales decrecientes, es decir que los elementos de salida que son el gasto total y las cuentas por pagar se encuentran controladas para el año 2015. En el segundo nivel se encuentran 8 DMU, lo que significa que se anticipan lecturas de problemas como se refleja en la siguiente tabla

Tabla 17 Eficiencia Financiera II Nivel

\begin{tabular}{|l|c|c|c|c|c|c|c|c|c|c|c|c|c|c|}
\hline AÑOS & $\mathbf{2 0 0 2}$ & $\mathbf{2 0 0 3}$ & $\mathbf{2 0 0 4}$ & $\mathbf{2 0 0 5}$ & $\mathbf{2 0 0 6}$ & $\mathbf{2 0 0 7}$ & $\mathbf{2 0 0 8}$ & $\mathbf{2 0 0 9}$ & $\mathbf{2 0 1 0}$ & $\mathbf{2 0 1 1}$ & $\mathbf{2 0 1 2}$ & $\mathbf{2 0 1 3}$ & $\mathbf{2 0 1 4}$ & $\mathbf{2 0 1 5}$ \\
\hline Bosa & 1,00 & 0,94 & 1,00 & 1,00 & 0,94 & 1,00 & 0,91 & 0,88 & 0,85 & 0,87 & 0,58 & 0,49 & 0,49 & 0,46 \\
\hline Centro Oriente & 0,86 & 0,81 & 0,89 & 0,84 & 0,69 & 0,70 & 0,93 & 0,83 & 0,81 & 0,91 & 0,83 & 0,79 & 0,88 & 0,81 \\
\hline Engativá & 0,77 & 0,85 & 0,85 & 1,00 & 0,84 & 1,00 & 0,87 & 0,90 & 0,82 & 0,98 & 0,99 & 0,77 & 0,74 & 0,85 \\
\hline Fontibón & 0,86 & 0,84 & 0,77 & 0,76 & 0,78 & 0,89 & 0,88 & 1,00 & 0,86 & 0,87 & 0,84 & 0,75 & 0,77 & 0,79 \\
\hline Meissen & 0,90 & 0,76 & 0,83 & 0,81 & 0,96 & 1,00 & 1,00 & 0,93 & 0,93 & 1,00 & 1,00 & 1,00 & 0,74 & 0,98 \\
\hline San Blas & 0,83 & 0,75 & 0,76 & 0,89 & 0,85 & 0,94 & 0,87 & 0,80 & 0,72 & 0,90 & 0,92 & 0,92 & 0,81 & 0,87 \\
\hline Suba & 1,00 & 0,79 & 0,78 & 0,76 & 0,83 & 0,90 & 0,84 & 1,00 & 0,89 & 0,94 & 0,96 & 0,88 & 0,84 & 0,90 \\
\hline Tunjuelito & 1,00 & 0,95 & 0,95 & 0,95 & 0,80 & 0,85 & 0,82 & 0,88 & 0,78 & 0,81 & 0,91 & 0,76 & 0,75 & 0,77 \\
\hline
\end{tabular}

Fuente: Elaborado por el autor con base en las salidas del modelo DEA desarrollado en Stata 2017 
En la siguiente figura se aprecia de mejor manera la trazabilidad por DMU durante el periodo objeto de estudio frente a la evaluación de eficiencia financiera

Figura 43 Eficiencia financiera II Nivel

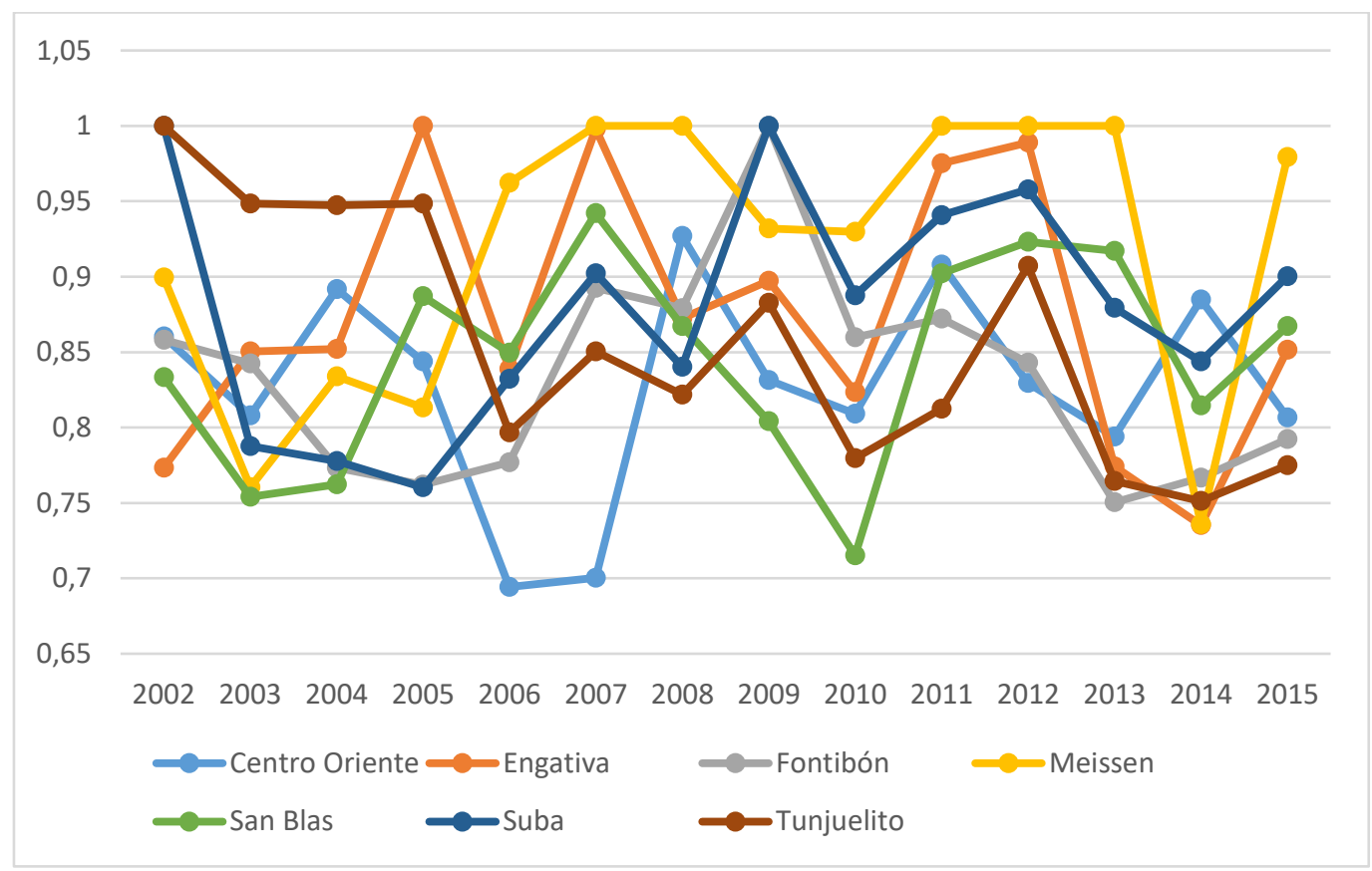

Fuente: Elaborado por el autor con base en las salidas del modelo DEA desarrollado en Stata 2017

Tabla 18 Eficiencia de gestión hospitalaria II Nivel

\begin{tabular}{|l|c|c|c|c|c|c|c|c|c|c|c|c|c|c|}
\hline AÑOS & $\mathbf{2 0 0 2}$ & $\mathbf{2 0 0 3}$ & $\mathbf{2 0 0 4}$ & $\mathbf{2 0 0 5}$ & $\mathbf{2 0 0 6}$ & $\mathbf{2 0 0 7}$ & $\mathbf{2 0 0 8}$ & $\mathbf{2 0 0 9}$ & $\mathbf{2 0 1 0}$ & $\mathbf{2 0 1 1}$ & $\mathbf{2 0 1 2}$ & $\mathbf{2 0 1 3}$ & $\mathbf{2 0 1 4}$ & $\mathbf{2 0 1 5}$ \\
\hline Bosa & 0,88 & 0,97 & 1,00 & 1,00 & 0,98 & 0,96 & 0,96 & 0,95 & 0,93 & 0,92 & 0,95 & 0,95 & 0,96 & 0,96 \\
\hline Centro Oriente & 0,94 & 0,93 & 0,94 & 0,91 & 0,91 & 0,87 & 0,90 & 0,90 & 0,86 & 0,88 & 0,89 & 0,86 & 0,85 & 0,84 \\
\hline Engativá & 0,87 & 0,86 & 0,85 & 0,80 & 0,76 & 0,75 & 0,75 & 0,75 & 0,76 & 0,71 & 0,70 & 0,69 & 0,74 & 0,76 \\
\hline Fontibón & 0,97 & 0,96 & 0,95 & 0,94 & 0,91 & 0,89 & 0,89 & 0,84 & 0,83 & 0,84 & 0,84 & 0,84 & 0,84 & 0,84 \\
\hline Meissen & 0,88 & 0,88 & 0,87 & 0,83 & 0,69 & 0,61 & 0,56 & 0,62 & 0,58 & 0,54 & 0,57 & 0,66 & 0,67 & 0,74 \\
\hline San Blas & 0,89 & 0,90 & 0,90 & 0,90 & 0,89 & 0,87 & 0,86 & 0,86 & 0,86 & 0,86 & 0,87 & 0,86 & 0,86 & 0,86 \\
\hline Suba & 1,00 & 0,96 & 0,92 & 0,89 & 0,80 & 0,76 & 0,74 & 0,68 & 0,64 & 0,64 & 0,64 & 0,60 & 0,62 & 0,65 \\
\hline Tunjuelito & 1,00 & 0,96 & 0,93 & 0,90 & 0,91 & 0,91 & 0,90 & 0,89 & 0,90 & 0,89 & 0,89 & 0,87 & 0,86 & 0,86 \\
\hline
\end{tabular}

Fuente: Elaborado por el autor con base en las salidas del modelo DEA desarrollado en Stata 2017

En la siguiente figura se aprecia de mejor manera la trazabilidad por DMU durante el periodo objeto de estudio frente a la evaluación de eficiencia financiera 


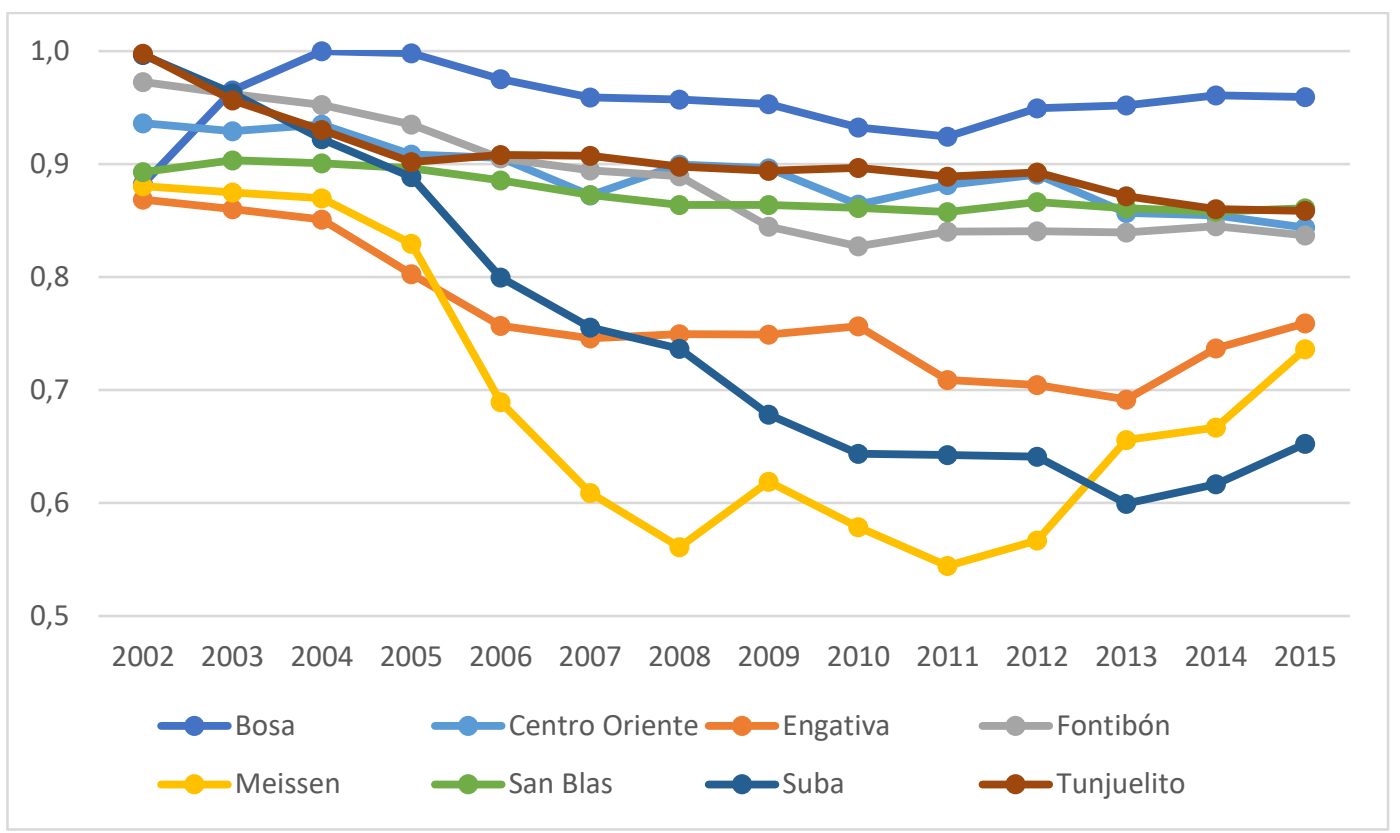

Fuente: Elaborado por el autor con base en las salidas del modelo DEA desarrollado en Stata 2017

La evaluación de eficiencia consolidada del II Nivel es la siguiente:

Tabla 19 Eficiencia consolidada II Nivel

\begin{tabular}{|l|r|r|r|r|r|r|r|r|r|r|r|r|r|r|}
\hline AÑOS & $\mathbf{2 0 0 2}$ & $\mathbf{2 0 0 3}$ & $\mathbf{2 0 0 4}$ & $\mathbf{2 0 0 5}$ & $\mathbf{2 0 0 6}$ & $\mathbf{2 0 0 7}$ & $\mathbf{2 0 0 8}$ & $\mathbf{2 0 0 9}$ & $\mathbf{2 0 1 0}$ & $\mathbf{2 0 1 1}$ & $\mathbf{2 0 1 2}$ & $\mathbf{2 0 1 3}$ & $\mathbf{2 0 1 4}$ & $\mathbf{2 0 1 5}$ \\
\hline Bosa & 0,94 & 0,95 & 1,00 & 1,00 & 0,96 & 0,98 & 0,93 & 0,92 & 0,89 & 0,90 & 0,77 & 0,72 & 0,73 & 0,71 \\
\hline Centro Oriente & 0,90 & 0,87 & 0,91 & 0,88 & 0,80 & 0,79 & 0,91 & 0,86 & 0,84 & 0,89 & 0,86 & 0,83 & 0,87 & 0,83 \\
\hline Engativa & 0,82 & 0,86 & 0,85 & 0,90 & 0,80 & 0,87 & 0,81 & 0,82 & 0,79 & 0,84 & 0,85 & 0,73 & 0,74 & 0,81 \\
\hline Fontibón & 0,92 & 0,90 & 0,86 & 0,85 & 0,84 & 0,89 & 0,88 & 0,92 & 0,84 & 0,86 & 0,84 & 0,80 & 0,81 & 0,81 \\
\hline Meissen & 0,89 & 0,82 & 0,85 & 0,82 & 0,83 & 0,80 & 0,78 & 0,78 & 0,75 & 0,77 & 0,78 & 0,83 & 0,70 & 0,86 \\
\hline San Blas & 0,86 & 0,83 & 0,83 & 0,89 & 0,87 & 0,91 & 0,87 & 0,83 & 0,79 & 0,88 & 0,89 & 0,89 & 0,84 & 0,86 \\
\hline Suba & 1,00 & 0,88 & 0,85 & 0,82 & 0,82 & 0,83 & 0,79 & 0,84 & 0,77 & 0,79 & 0,80 & 0,74 & 0,73 & 0,78 \\
\hline Tunjuelito & 1,00 & 0,95 & 0,94 & 0,93 & 0,85 & 0,88 & 0,86 & 0,89 & 0,84 & 0,85 & 0,90 & 0,82 & 0,81 & 0,82 \\
\hline
\end{tabular}

Fuente: Elaborado por el autor con base en las salidas del modelo DEA desarrollado en Stata 2017

En la siguiente figura se aprecia de mejor manera la trazabilidad por DMU durante el periodo objeto de estudio frente a la evaluación de eficiencia financiera 


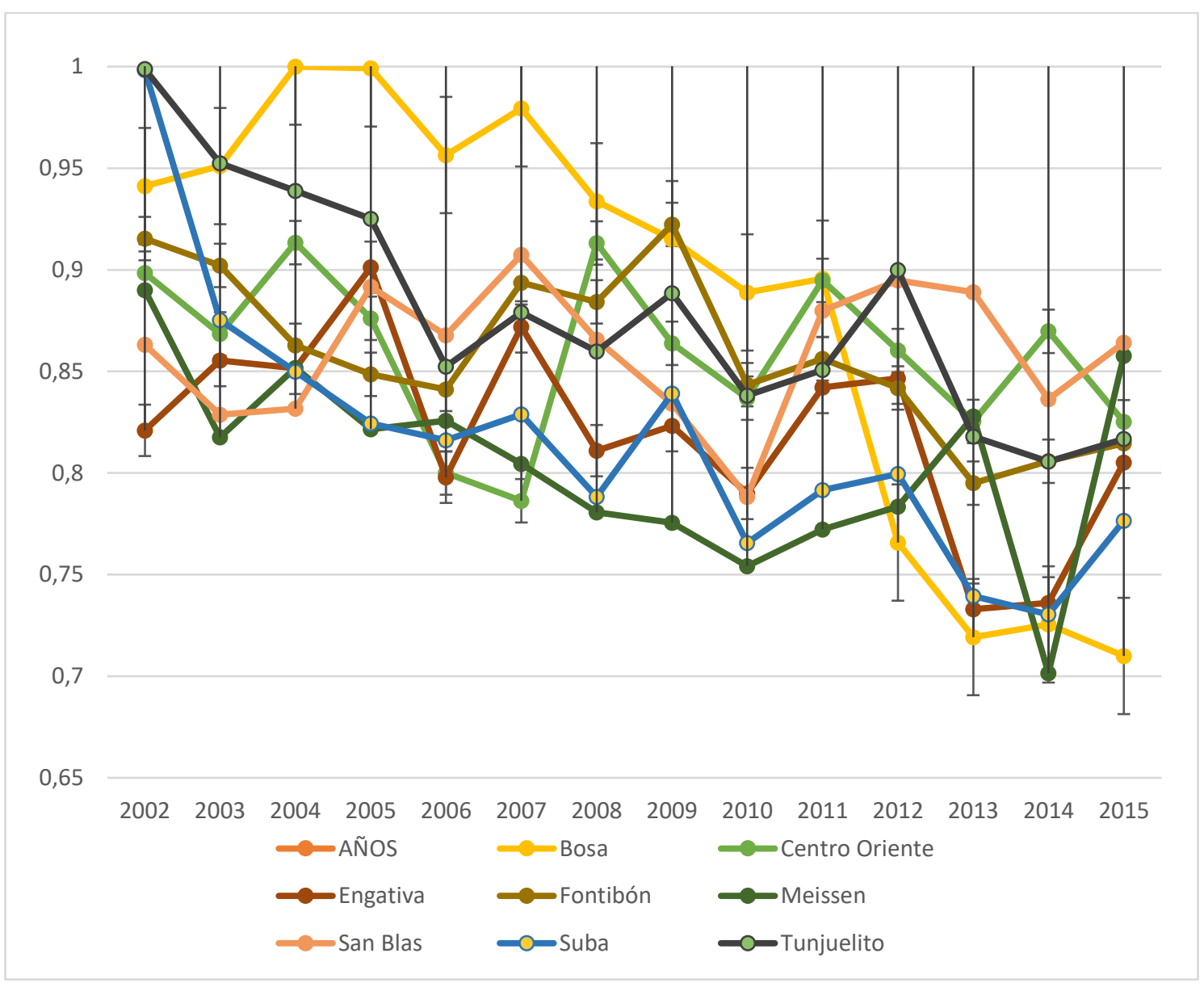

Fuente: Elaborado por el autor con base en las salidas del modelo DEA desarrollado en Stata 2017

Se evidencia una pérdida sistemática en el II Nivel de atención, sin embargo, la DMU con mayores pérdidas de eficiencias es el Hospital de Bosa, hospital que pasa de hacer parte del límite superior de la banda a la parte inferior de la misma

\subsubsection{Resultados III Nivel}

La evaluación de eficiencia financiera se resume en la siguiente tabla

Tabla 20 Eficiencia financiera III Nivel

\begin{tabular}{|l|c|c|c|c|c|c|c|c|c|c|c|c|c|c|}
\hline AÑOS & $\mathbf{2 0 0 2}$ & $\mathbf{2 0 0 3}$ & $\mathbf{2 0 0 4}$ & $\mathbf{2 0 0 5}$ & $\mathbf{2 0 0 6}$ & $\mathbf{2 0 0 7}$ & $\mathbf{2 0 0 8}$ & $\mathbf{2 0 0 9}$ & $\mathbf{2 0 1 0}$ & $\mathbf{2 0 1 1}$ & $\mathbf{2 0 1 2}$ & $\mathbf{2 0 1 3}$ & $\mathbf{2 0 1 4}$ & $\mathbf{2 0 1 5}$ \\
\hline Kennedy & 0,95 & 0,91 & 0,88 & 0,94 & 0,85 & 1,00 & 1,00 & 0,99 & 1,00 & 0,92 & 0,96 & 0,87 & 0,93 & 1,00 \\
\hline La Victoria & 1,00 & 0,96 & 1,00 & 0,93 & 0,98 & 1,00 & 1,00 & 0,97 & 0,99 & 1,00 & 1,00 & 1,00 & 0,94 & 1,00 \\
\hline Santa Clara & 1,00 & 0,86 & 0,86 & 0,95 & 0,95 & 0,83 & 0,98 & 0,86 & 0,99 & 0,98 & 0,88 & 0,80 & 0,80 & 0,88 \\
\hline Simón Bolívar & 0,93 & 0,84 & 0,89 & 0,95 & 1,00 & 0,91 & 0,87 & 0,90 & 0,93 & 0,99 & 0,96 & 0,97 & 0,99 & 1,00 \\
\hline Tunal & 1,00 & 0,86 & 0,92 & 0,89 & 0,98 & 0,89 & 0,88 & 1,00 & 1,00 & 0,86 & 1,00 & 0,86 & 1,00 & 1,00 \\
\hline
\end{tabular}

Fuente: Elaborado por el autor con base en las salidas del modelo DEA desarrollado en Stata 2017 
En la siguiente figura se aprecia de mejor manera la trazabilidad por DMU durante el periodo objeto de estudio frente a la evaluación de eficiencia financiera.

Figura 46 Eficiencia financiera III nivel

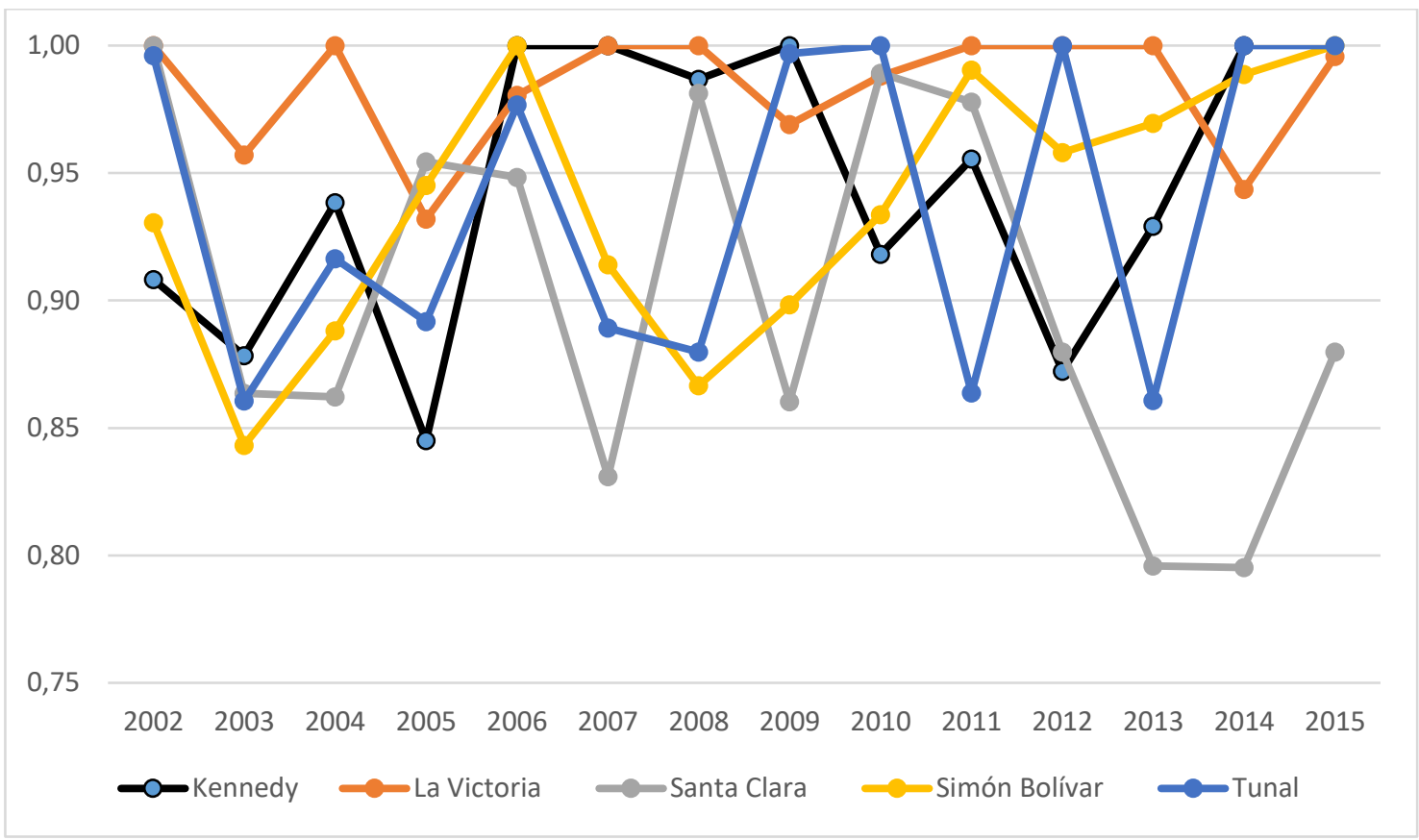

Fuente: Elaborado por el autor con base en las salidas del modelo DEA desarrollado en Stata 2017

La eficiencia financiera se espera tenga rendimientos decrecientes, es decir que aumenten los ingresos y disminuyan las cuentas por pagar, hay tres hospitales que para 2015, logran ajustes importantes y por ello convergen a la parte superior de la tabla, el hospital que ostenta la mayor regularidad en la perspectiva financiera es el Hospital la Victoria, del cual podemos recordar que no estuvo obligado a someterse a programas de saneamiento fiscal y financiero por no haber sido evaluado con riesgo alto o medio; el hospital Santa Clara por el contrario se ubica como el Hospital con menor eficiencia financiera al final del periodo observado.

Tabla 21 Eficiencia hospitalaria III Nivel

\begin{tabular}{|l|c|c|c|c|c|c|c|c|c|c|c|c|c|c|}
\hline AÑOS & $\mathbf{2 0 0 2}$ & $\mathbf{2 0 0 3}$ & $\mathbf{2 0 0 4}$ & $\mathbf{2 0 0 5}$ & $\mathbf{2 0 0 6}$ & $\mathbf{2 0 0 7}$ & $\mathbf{2 0 0 8}$ & $\mathbf{2 0 0 9}$ & $\mathbf{2 0 1 0}$ & $\mathbf{2 0 1 1}$ & $\mathbf{2 0 1 2}$ & $\mathbf{2 0 1 3}$ & $\mathbf{2 0 1 4}$ & $\mathbf{2 0 1 5}$ \\
\hline Kennedy & 0,99 & 1,00 & 0,99 & 0,97 & 0,93 & 0,91 & 0,88 & 0,83 & 0,78 & 0,80 & 0,81 & 0,80 & 0,80 & 0,76 \\
\hline La Victoria & 0,92 & 1,00 & 1,00 & 0,95 & 0,92 & 0,84 & 0,83 & 0,81 & 0,78 & 0,68 & 0,65 & 0,61 & 0,67 & 0,63 \\
\hline Santa Clara & 0,92 & 0,92 & 0,91 & 0,98 & 0,97 & 1,00 & 0,98 & 0,92 & 0,85 & 0,84 & 0,84 & 0,84 & 0,87 & 0,86 \\
\hline Simón Bolívar & 0,99 & 0,97 & 0,96 & 0,92 & 0,85 & 0,86 & 0,86 & 0,85 & 0,82 & 0,75 & 0,76 & 0,74 & 0,75 & 0,80 \\
\hline Tunal & 1,00 & 0,97 & 0,97 & 0,95 & 0,94 & 0,94 & 0,92 & 0,90 & 0,83 & 0,86 & 0,89 & 0,85 & 0,82 & 0,80 \\
\hline
\end{tabular}

Fuente: Elaborado por el autor con base en las salidas del modelo DEA desarrollado en Stata 2017 
En la siguiente figura se aprecia de mejor manera la trazabilidad por DMU durante el periodo objeto de estudio frente a la evaluación de eficiencia financiera.

Figura 47 Evaluación de eficiencia hospitalaria

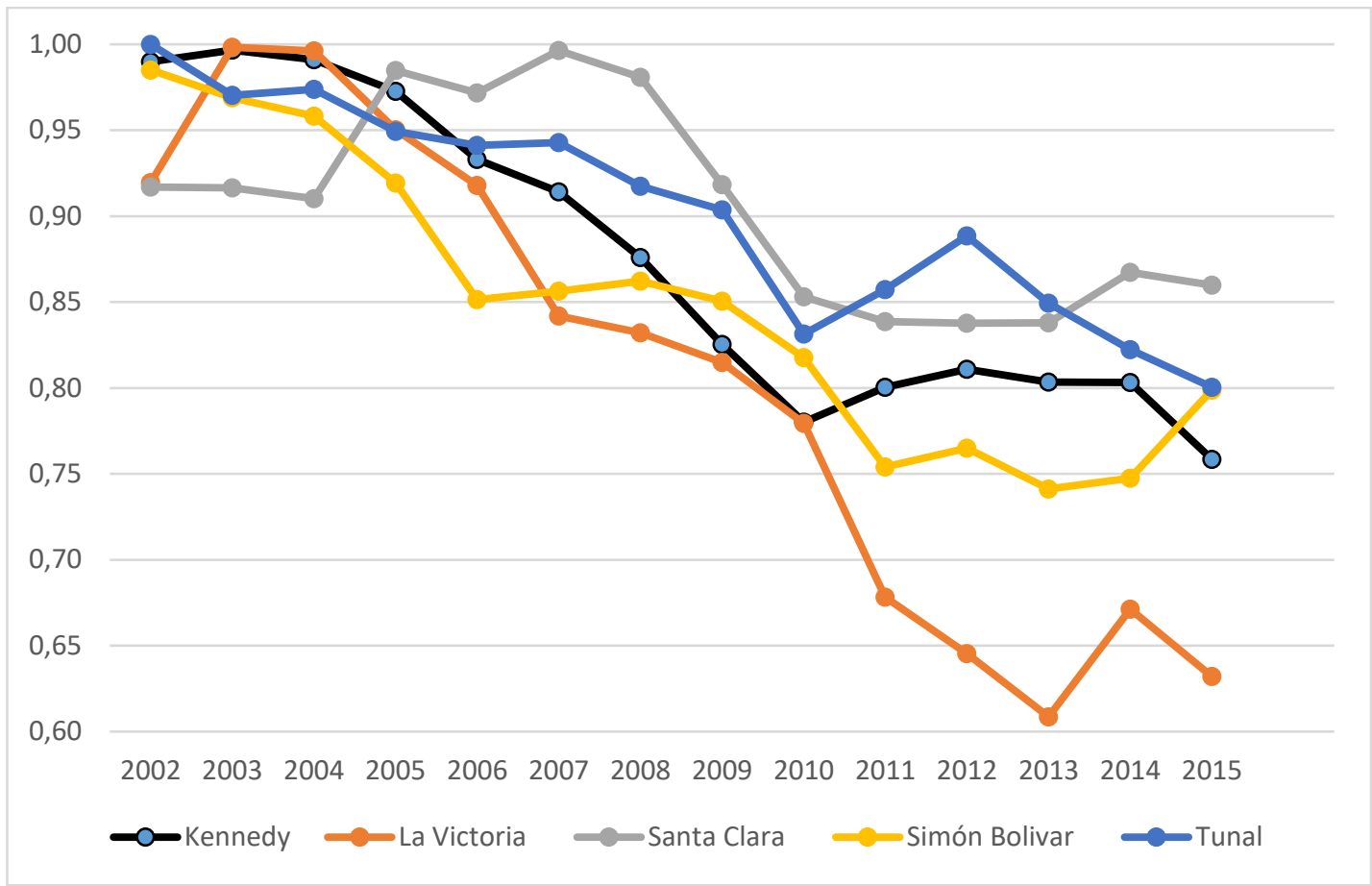

Fuente: Elaborado por el autor con base en las salidas del modelo DEA desarrollado en Stata 2017

La eficiencia consolidada del tercer nivel es la siguiente

Tabla 22 Evaluación de eficiencia III Nivel

\begin{tabular}{|l|r|r|r|r|r|r|r|r|r|r|r|r|r|r|}
\hline AÑOS & $\mathbf{2 0 0 2}$ & $\mathbf{2 0 0 3}$ & $\mathbf{2 0 0 4}$ & $\mathbf{2 0 0 5}$ & $\mathbf{2 0 0 6}$ & $\mathbf{2 0 0 7}$ & $\mathbf{2 0 0 8}$ & $\mathbf{2 0 0 9}$ & $\mathbf{2 0 1 0}$ & $\mathbf{2 0 1 1}$ & $\mathbf{2 0 1 2}$ & $\mathbf{2 0 1 3}$ & $\mathbf{2 0 1 4}$ & $\mathbf{2 0 1 5}$ \\
\hline Kennedy & 0,97 & 0,95 & 0,93 & 0,96 & 0,89 & 0,96 & 0,94 & 0,91 & 0,89 & 0,86 & 0,88 & 0,84 & 0,87 & 0,88 \\
\hline La Victoria & 0,96 & 0,98 & 1,00 & 0,94 & 0,95 & 0,92 & 0,92 & 0,89 & 0,88 & 0,84 & 0,82 & 0,80 & 0,81 & 0,81 \\
\hline Santa Clara & 0,96 & 0,89 & 0,89 & 0,97 & 0,96 & 0,91 & 0,98 & 0,89 & 0,92 & 0,91 & 0,86 & 0,82 & 0,83 & 0,87 \\
\hline Simón Bolívar & 0,96 & 0,91 & 0,92 & 0,93 & 0,93 & 0,89 & 0,86 & 0,87 & 0,88 & 0,87 & 0,86 & 0,86 & 0,87 & 0,90 \\
\hline Tunal & 1,00 & 0,92 & 0,95 & 0,92 & 0,96 & 0,92 & 0,90 & 0,95 & 0,92 & 0,86 & 0,94 & 0,86 & 0,91 & 0,90 \\
\hline
\end{tabular}

Fuente: Elaborado por el autor con base en las salidas del modelo DEA desarrollado en Stata 2017

En la siguiente figura se aprecia de mejor manera la trazabilidad por DMU durante el periodo objeto de estudio frente a la evaluación de eficiencia consolidada. 


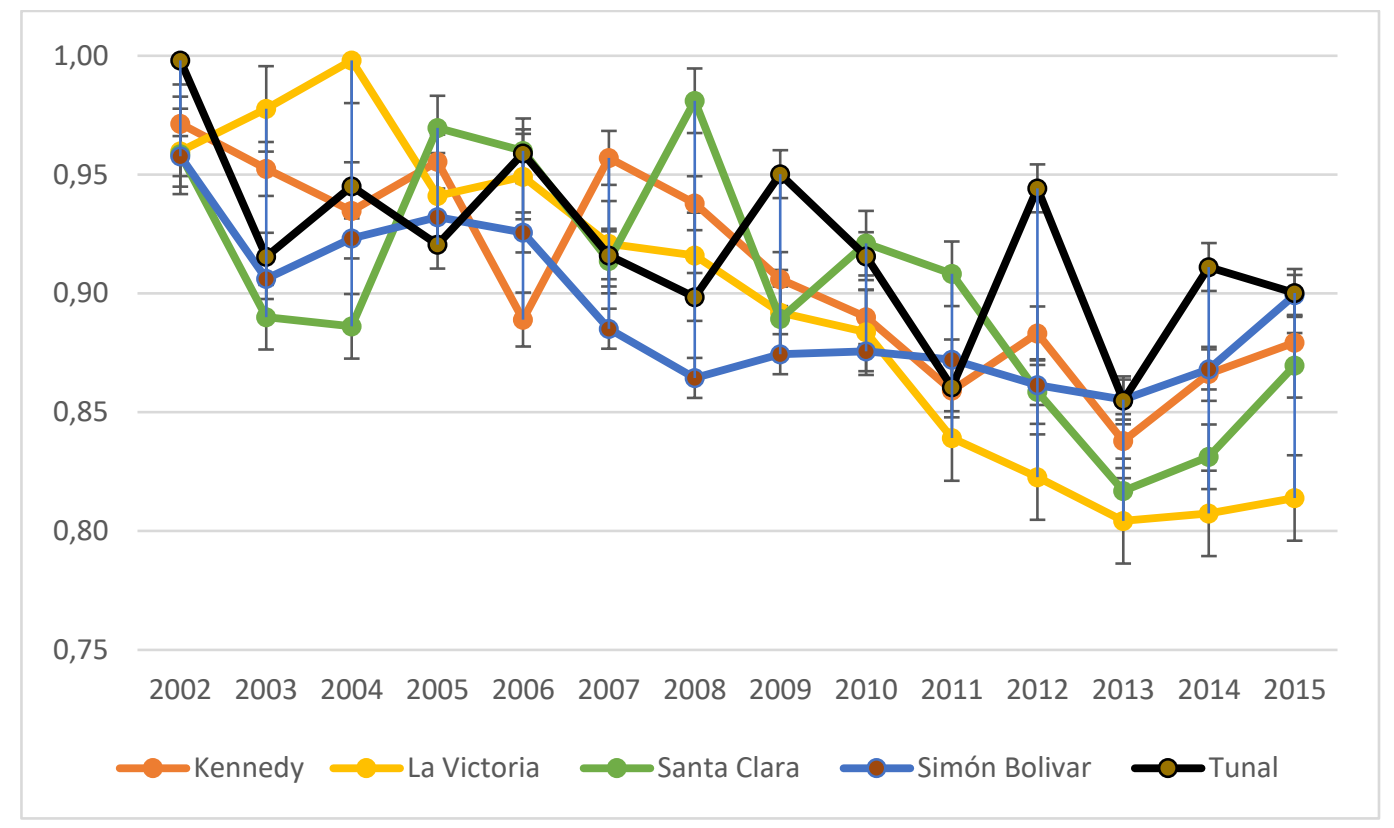

Fuente: Elaborado por el autor con base en las salidas del modelo DEA desarrollado en Stata 2017

En el III Nivel la evaluación de eficiencia se aprecia como una brecha que con tendencia a la disminución sistemática de la eficiencia en concordancia principalmente con la evaluación de la eficiencia hospitalaria, sin embargo, aunque en la figura global de eficiencia financiera no se identifica claramente una tendencia, si se advierte un comportamiento con pérdida de eficiencia con una mejora sustantiva sobre el final gracias a los programas de saneamiento fiscal y financiero que termina premiando al Hospital Simón Bolívar con una eficiencia conjugada equivalente a 0,9 , con la que logra compartir el primer lugar con el Hospital el Tunal.

\subsubsection{Propuesta de intervención para mejorar la eficiencia y productividad en la prestación de los servicios de la red hospitalaria de Bogotá, a partir de los resultados logrados en la evaluación de la eficiencia de la red pública hospitalaria}

De la situación de optimización de las unidades hospitalarias por Nivel de Atención se evidencia una necesidad de articular la política pública, la demanda insatisfecha por servicios de salud de mediana y alta complejidad, la salud como derecho fundamental y la captura del regulador (al que hay que despertar) con el fin de estabilizar la viabilidad 
de la salud pública comprometida por la permanente queja de no pago de los servicios prestados a los usuarios y facturados a las EPS.

Resulta de la mayor conveniencia para los prestadores del servicio mejorar la certeza de pago sobre los servicios en virtud de que la cartera a precios constantes no ha parado de crecer durante los años observados, con el agravante de que muchas EPS se han declarado en quiebra y han abandonado el mercado, desangrando el sistema.

Es indispensable que el gobierno nacional se concentre en fortalecer las reglas de juego de tal forma que se priorice y garantice el flujo de recursos mediante subsidios a la oferta copiando mecanismos como los propuestos en el capítulo de desarrollo, una cámara de compensación y apoyando mediante el sistema general de participaciones la contratación "fundamental" de la mano de obra hospitalaria o en su defecto de un porcentaje.

La mano de obra en los hospitales no se refleja de forma acertada en las ejecuciones presupuestales debido a que los gastos de nómina y asociados representan porcentajes diferentes en cada una de las ESE respecto de los contratos administrativos por prestación de servicios, sin embargo, en caso de que se conviniera aprobar un porcentaje de la contratación de la mano de obra de los hospitales, se requeriría también cambiar el flujo de los recursos para establecer un subsidio a la oferta, diferente a la forma con la cual se opera a partir de la implementación de la Ley 100.

Finalmente resulta de la mayor importancia ampliar los servicios de mayor complejidad lo cual es imposible sin contar con una planeación de consenso entre los actores, controlar la corrupción y establecer acceso igualitario para despertar al regulador de su estado de confort.

Se advierte que el sistema general de seguridad social en salud requiere de un servicio de calidad y sostenible a los usuarios, para lo cual, se pueden revisar modelos de funcionamiento como por el ejemplo en del sector financiero en el cual, el Banco de la República funciona como una cámara de compensación.

La definición de compensación, tomado del Reglamento Operativo del Servicio de Compensación Interbancaria del Banco de La República dice que es "Un sistema que permite a las entidades Autorizadas compensar y/o liquidar instrucciones de pago propias o de terceros realizadas a través de los Instrumentos de Pago. Se trata de facilitar y 
garantizar el intercambio, pagos y todo tipo de transacciones entre entidades afiliadas en las que se conocen perfectamente los integrantes con el fin de reducir el riesgo de los miembros que puedan incumplir con sus obligaciones comerciales.

Los cámaras de compensación han existido durante mucho tiempo y se han utilizado para muchos tipos de transacciones, sin embargo, prácticamente todas las cámaras de compensación desempeñan ciertas funciones básicas. En particular, al centralizar y estandarizar clases específicas de transacciones financieras, las cámaras de compensación reducen los costos y los riesgos operacionales de compensación y liquidación entre múltiples participantes en el mercado. En muchos casos, también actúan como garantes de las transacciones, lo que ayuda a reducir el riesgo de contraparte de crédito y liquidez.

Debido a que la falla o pérdida de confianza en una cámara de compensación principal crearía una enorme incertidumbre sobre el estado de las transacciones se requiere una supervisión eficaz sobre las transacciones y posiciones financieras de los participantes y sus clientes, una fuerte gestión de riesgos en estas organizaciones.

Una perspectiva histórica es útil para comprender el funcionamiento de las cámaras de compensación y las implicaciones de sus operaciones para la estabilidad financiera. La primera cámara de compensación importante se instauró en los Estados Unidos, la Cámara de Compensación de Nueva York, fue fundada por los bancos comerciales de la ciudad de Nueva York en 1853 para agilizar la liquidación y transparencia en el pago de cheques (Gibbons, 1857).

En una cuenta, antes de que se estableciera la Cámara de Compensación de Nueva York, el proceso de liquidación involucraba a empleados de 60 bancos cruzando sus cuentas por las calles de la ciudad para presentar cheques, un proceso que llevaba mucho tiempo lleno de "confusión, disputas y equivocaciones inevitables". "El establecimiento del centro de intercambio de información mejoró la situación casi de inmediato, lo que permitió ahorros significativos en tiempo, esfuerzo y costos financieros. A fines del siglo XIX, se habían establecido múltiples cámaras de compensación (National Monetary Commission, 1910). 
Para el caso de los mercados de valores y derivados, la historia se desarrolló un poco más tarde y fue si se quiere más compleja que en el caso de los cheques. Sin embargo, muchos de los temas esenciales son los mismos. En 1892, la Bolsa de Valores de Nueva York dio sus primeros pasos para mejorar la liquidación de transacciones creando una cámara de compensación para los intercambios ante el creciente volumen de transacciones y los costos asociados. Después de la Primera Guerra Mundial, la Bolsa de Nueva York dio un paso más en 1920 al establecer la "Stock Clearing Corporation"; esta nueva cámara de compensación redujo el número de verificaciones necesarias para el cierre de transacciones hasta en un 90 por ciento y el volumen de fondos y créditos necesarios en un 70 por ciento o más (National Monetary Commission, 1910).

El papel de las cámaras de compensación se puede entender de dos maneras diferentes. En primer lugar, su objetivo es aumentar la resiliencia de las instituciones frente a crisis financieras graves. En segundo lugar, también alienta un mayor desarrollo y uso de las cámaras de compensación de intercambio de información para abordar las deficiencias identificadas en otras partes del sistema general de seguridad social en salud. Por supuesto, una mayor dependencia de las cámaras de compensación para abordar los problemas en otras partes del sistema aumenta aún más la necesidad de garantizar la seguridad y estabilidad de las cámaras de compensación.

Luego de un acuerdo jurídicamente vinculante en la ejecución repetitiva de intercambio entre un comprador y un vendedor, la función de la cámara de compensación es centralizar y estandarizar todos los pasos previos al pago de la transacción con consecuencias positivas sobre la disminución de costos de transacción, riesgo operacional, riesgo de crédito y liquidaciones múltiples con la garantía que requieren los juegos repetitivos.

Si los vendedores temieran que los compradores no van a pagar en el futuro, lo más seguro es que el mercado sufriera una contracción de sus operaciones; la salud que no funciona como un mercado competitivo, permite una posición dominante para los compradores que realmente operan como pagadores de servicios, esta situación se presta para no reconocer o dilatar los servicios facturados por parte de las IPS. 
Eliminando las asimetrías sobre la participación de los recursos del sistema, en el que los pagadores puedan estar seguros del servicio que se les presta a los usuarios y los prestadores tengan un alto grado de certeza sobre el ingreso de los recursos por los servicios prestados se espera que las ESE puedan concentrarse en prestar un servicio de calidad y los intermediarios financieros en garantizar las coberturas prometidas.

\section{CONCLUSIONES GENERALES}

El análisis de eficiencia de la Red Pública de hospitales del Distrito capital durante el periodo 2002-2015 muestra que en general los hospitales experimentan un deterioro de la eficiencia de gestión financiera y de gestión hospitalaria componentes presentados para medir la eficiencia general de las ESE.

Se concluye que los modelos de gobernanza de las IPS (DMU) afectan el desempeño en los niveles de atención II y III que se traduce en una pérdida general de eficiencia de la red pública hospitalaria de Bogotá D.C. durante el periodo de estudio, lo cual afecta la creciente demanda por servicios de salud sin reacción o limitada reacción por parte de la oferta de camas y de servicios de la red pública hospitalaria.

La forma de responder a la pregunta de investigación se asocia a la evaluación de la eficiencia que fue medida por grupos homogéneos de hospitales, ya que las instituciones fueron agrupadas por grado de complejidad en 3 niveles; de esta forma se advierte una pérdida sistemática de eficiencia en los hospitales de II y III nivel de complejidad y al interior de los grupos se encuentran Hospitales que jalonan mayormente el comportamiento, tal como se muestra en el capítulo III.

La disminución de la productividad se debe revisar detenidamente porque, aunque se trata de modelar escogiendo las mejores variables posibles, la complejidad del sistema general de seguridad social en salud es muy amplia para lograr capturar las particularidades que afectan la red pública de hospitales del Distrito Capital

Existe una inflación normativa que no se cumple en materia de proceso administrativo relacionado con la recuperación de la cartera; sobre este particular se han llevado a cabo mesas de concertación con las EPS a las que asisten delegados sin poder de toma de 
decisiones para de manera sistemática incumplir lo firmado, situación que ha redundado en el no pago de la cartera hospitalaria.

De acuerdo con el marco de referencia un cambio en lo público motiva a los privados para adoptar o desarrollar cambios en la misma dirección, en este momento resulta de la mayor importancia seguir en el camino de la disminución de barreras de acceso y lograr la estabilidad financiera de los prestadores de servicio, porque, aunque no es objeto de estudio del presente documento, si se ha disparado la demanda de servicios de salud en el sector privado y una mejora ostensible es mejorar la cobertura de los servicios de mayor complejidad.

El modelo no paramétrico de Análisis envolvente de datos por niveles de complejidad muestra que a mayor grado de complejidad, mayor castigo de la cartera lo cual establece una relación inversa que resulta explosiva frente a la sostenibilidad de las ESE y del sistema general de seguridad social en salud que deriva en una pérdida de eficiencia, motivo por el cual son más eficientes los hospitales de menor grado de complejidad, esto señala los requerimientos relacionados con la distribución de los recursos.

Existe evidencia según el modelo de análisis envolvente de datos que muestra una pérdida sistemática de eficiencia en los niveles de complejidad II y III. En el II nivel los hospitales de Suba, Meissen y Engativá, han presentado desde el año 2007 una baja eficiencia de gestión hospitalaria, sin descartar que la eficiencia financiera también representa problemas, sin embargo, se considera que las deficiencias financieras son un riesgo no sistemático para las ESE y se concluye que existe un importante grado de asociación entre la eficiencia y el grado de complejidad de los servicios.

En el I Nivel no se advierte que la pérdida de eficiencia tenga correspondencia con una tendencia, por el contrario, se puede apreciar que existe una brecha importante entre las diferentes DMU, esto se puede explicar por las diversas poblaciones que deben atender en áreas de influencia distintas que representan problemas y necesidades diferentes.

Se debe desfavorecer la competencia entre los hospitales en la conformación de las redes, pero este propósito requiere la modernización de factores administrativos que permitan 
la complementariedad de las de las relaciones con el fin de mejorar el desarrollo y compromiso del talento humano con el servicio y no con las finanzas.

Existe un riesgo propio del modelo al tomar como información de salida la producción en UVR y tiene que ver con la medida de los resultados sin observancia alguna de los procesos, es decir que al parecer los pacientes se atienden de forma homogénea, cuando se sabe que aunque los diagnósticos puedan ser iguales, los tratamientos y los tiempos de respuesta son diferentes, lo cual dificulta el establecimiento de parámetros de calidad que no se tuvieron en cuenta en el modelo.

La caída de la eficiencia sugiere la necesidad de una evaluación del sistema de hospitales públicos ampliando la cantidad de DMU y poder tomar un benchmark más amplio y consistente para proponer lineamientos de mejora generales que definan los logros y objetivos cuando se comparte un mismo sistema y corregir las ineficiencias con una propuesta de cambios en las políticas planteadas por el gobierno sin perder los propósitos de calidad y accesibilidad de los servicios.

Se requiere de compromiso por parte de las EPS para el pago oportuno de las obligaciones con las IPS situación que se propone se resuelva con la implementación de una cámara de compensación para el SGSSS ya que de otra forma el esquema de subsidios a la demanda condenará el sistema. 


\section{BIBLIOGRAFÍA}

Almula Karadayi, M., \& Karsak , E. (2014). IMPRECISE DEA FRAMEWORK FOR EVALUATING THE EFFICIENCY OF STATE HOSPITALS IN ISTANBUL. Proceedings of the World Congress on Engineering, 1-6.

Arrow, K. (1951). Social Choice and Individual Values. New York: John Wiley \& Sons, Inc.

Barro, R., Blinder, A., \& Nordhaus, W. (1984). Discussion. The American Economic Review, 416422.

Bhat, R., Bhushan Verma, B., \& Reuben, E. (2001). AN EMPIRICAL ANALYSIS OF DISTRICT HOSPITALS AND GRANT-IN-AID HOSPITALS IN GUJARAT STATE OF INDIA. Indian Institute of Management, 2-40.

Canay, I. A. (2001). Fronteras de Eficiencia Estocásticas. Seminario de Integración y Aplicación, Licenciatura en Economía, 1-30.

Charnes, A., Cooper , W., \& Rhodes, E. (November 1978). Measuring the efficiency of decision making units. European Journal of Operational Research.

Coelli, T., Estanche, A., Perelman, S., \& Trujillo, L. (2003). Una introducción a las medidas de eficiencia para reguladores de servicios públicos y de transporte. Bogotá: Banco Mundial - Alfa Omega. 
Congreso de la República de Colombia. (23 de diciembre de 1993). Ley 100 de 1993. "Por la cual se crea el sistema de seguridad social integral y se dictan otras disposiciones"(Artículo 194). Bogotá D.C., República de Colombia.

Congreso de la República de Colombia. (2006). Ley 1116.

Courgeau, D. (1999). Métodos para análisis de datos biográficos. Estudios Demográficos y Urbanos, 14(3), 599-629. Recuperado el 19 de 11 de 2017, de http://aleph.academica.mx/jspui/bitstream/56789/21673/1/14-042-1999-0599.pdf

Flórez Pinilla, K. (2010). La competencia regulada en sistema de salud colombiano. Revista Lebret.

Franco Giraldo, A. (2011). Ley 1438 de 2011: Entre superflua y disuasiva. bibliotecadigital.udea.edu.co.

Goldratt, E., \& Cox, J. (1998). La Meta, un proceso de mejora continua. México: Ediciones Castillo.

González , J. I. (1998). Amartya Sen y la Elección Social. Cuadernos de Economía Vol. 17, 163184.

González, J. I. (2004). The dimensión of the reasonable in microeconomics of William Vickrey. Cuadernos de Economía, 50-85.

Kawaguchi, H., Tone, K., \& Tsutsui, M. (2013). ESTIMATION OF THE EFFICIENCY OF JAPANESE HOSPITALS USING A DYNAMIC AND NETWORK DATA ENVELOPMENT ANALYSIS MODEL. Workshop on DNDEA, 65-72.

Maldonado, N., \& Tamayo, A. (2007). Estudio Integral de Eficiencia de los Hospitales Públicos. Archivos de Economía, Departamento Nacional de Planeación, Dirección de Estudios Económicos, 1-64.

Meza Carvajalino, C. A., \& Isaza Castro, J. (29 de junio de 2005). Crecimiento económco, empleo formal y acceso al servicio de salud: Algunos escenarios de corto y largo plazo de la cobertura en salud para Colombia. (U. d.-D.-C. social-CIDES, Ed.) Archivo de economía(288).

Nicolson, W. (2008). Teoria Microeconomica. Mexico D.F.: Cengage Learning Editores S.A.

North, D. (1995). Instituciones, cambio institucional y desempeño económico. México: Fondo de Cultura Económica.

Nupia, O. A., \& Sánchez, F. (2001). Eficiencia de los hospitales públicos de Bogotá. Desarrollo y Sociedad, 101-136.

O Connor, J. (2002). La Crisis Fiscal del Estado. Barcelona: Edición 62. 
Orozco Gallo, A. J. (2014). Una aproximación regional a la eficiencia y productividad de los hospitales públioc colombianos. Banco de la República, Centro de estudios regionales (CEER) - Cartagena, 1-53.

Peña, M. L., Marroquín, M. G., \& Ríos, M. C. (2008). Optimización multicriterio por análisis envolvente de datos: Estrategias de agrupamiento y discriminación. Recuperado el 19 de 11 de 2017, de http://ingenierias.uanl.mx/38/38_optimizacion.pdf

Pestana Barros , C., Gomes de Menezes, A., Cabral Vieira, J., Peypoch, N., \& Solonandrasana , B. (2007). AN ANALYSIS OF HOSPITAL EFFICIENCY AND PRODUCTIVITY GROWTH USING THE LUENBERGER PRODUCTIVITY INDICATOR. DISCUSSION PAPER SERIES, 3-13.

Pinzón, M. (2003). Medición de eficiencia técnica relativa en hospitales públicos de baja complejidad mediante la metodología DATA Envelopment Enalysis(DEA). Bogotá: Universidad Javeriana .

Post, T., \& Spronk, J. (1999). Performance benchmarking using interactive data envelopment analysis. European Journal of Operational Research, 115-472.

Rawls, J. (1971). Teoría de la Justicia. FCE.

Real Academia Española. (4 de 12 de 2016). Asociación de Academias de la Lengua Española . Obtenido de http://dle.rae.es/?id=UH8mXZv

Rodríguez R., L. (2008). Derecho Administrativo General y Colombiano. Bogotá - Colombia: Editorial Temis S.A.

Rousseau, J. (1755). Discurso sobre el origen de la desigualdad entre los hombres. (E. d. Sociales, Ed.) Obras escogidas de Rosseau.

Salud Capital. (25 de 07 de 2017). Salud Capital. Obtenido de http://saludcapital.gov.co/Documents/Red\%20Prestadora\%20de\%20Servicios\%20de\% 20Salud\%20-\%20Bogota\%20-\%202015-2016.pdf

Secretaría Distrital de Salud. (2015). Diagnóstico sectorial de salud. Bogotá D.C.

Secretaría Distrital de Salud. (2015). Informe de seguimiento al programa de saneamiento fiscal y financiero I Semestre 2014. Bogotá D.C.

Sentencia T-121/15, Expediente T-4.574.405 (Corte Constitucional 26 de marzo de 2015).

Stigler, G. J. (1961). The Economics of Information. The Journal of Political Economy, 213-225.

Varian, H. R. (1999). Microecnomía Intermedia, un enfoque actual. Barcelona: Alfaomega.

Vickrey, W. (1939). Averagin of income-tax purpuses. Journal of Political Economy, 379-397.

Vickrey, W. (1948). Economics of public finance. American Economic Review, 172-175. 
Wiesner, E. (1998). La Efectividad de las Políticas Públicas en Colombia: Un análisis neoinstitucional. Bogotá D.C.: Tercer mundo editores; Departamento Nacional de Planeación. 


\section{ANEXOS}

Anexo 1 Tabla de salida perspectiva financiera I Nivel.

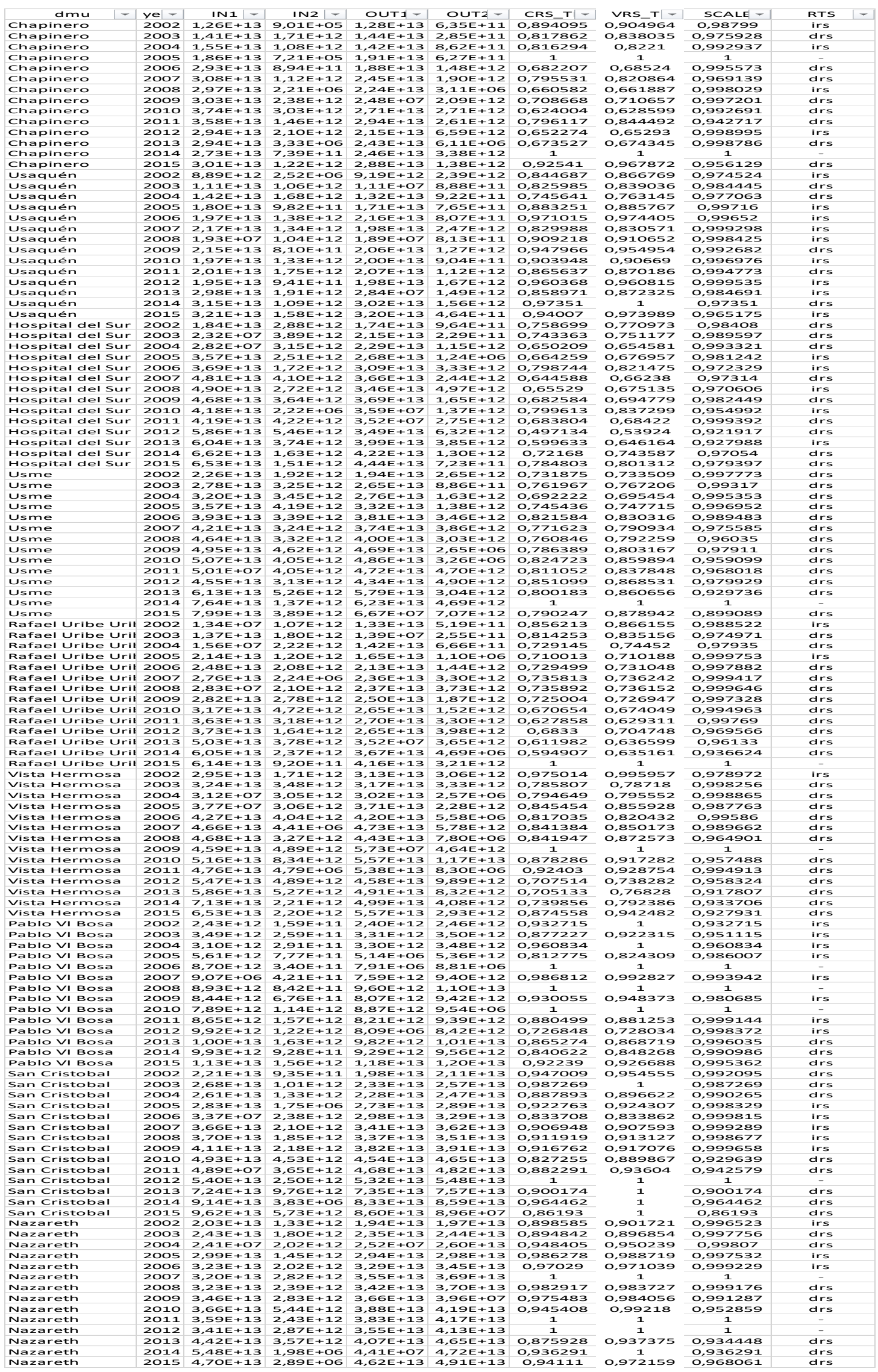


Anexo 2 Tabla de salidas perspectiva científica I Nivel

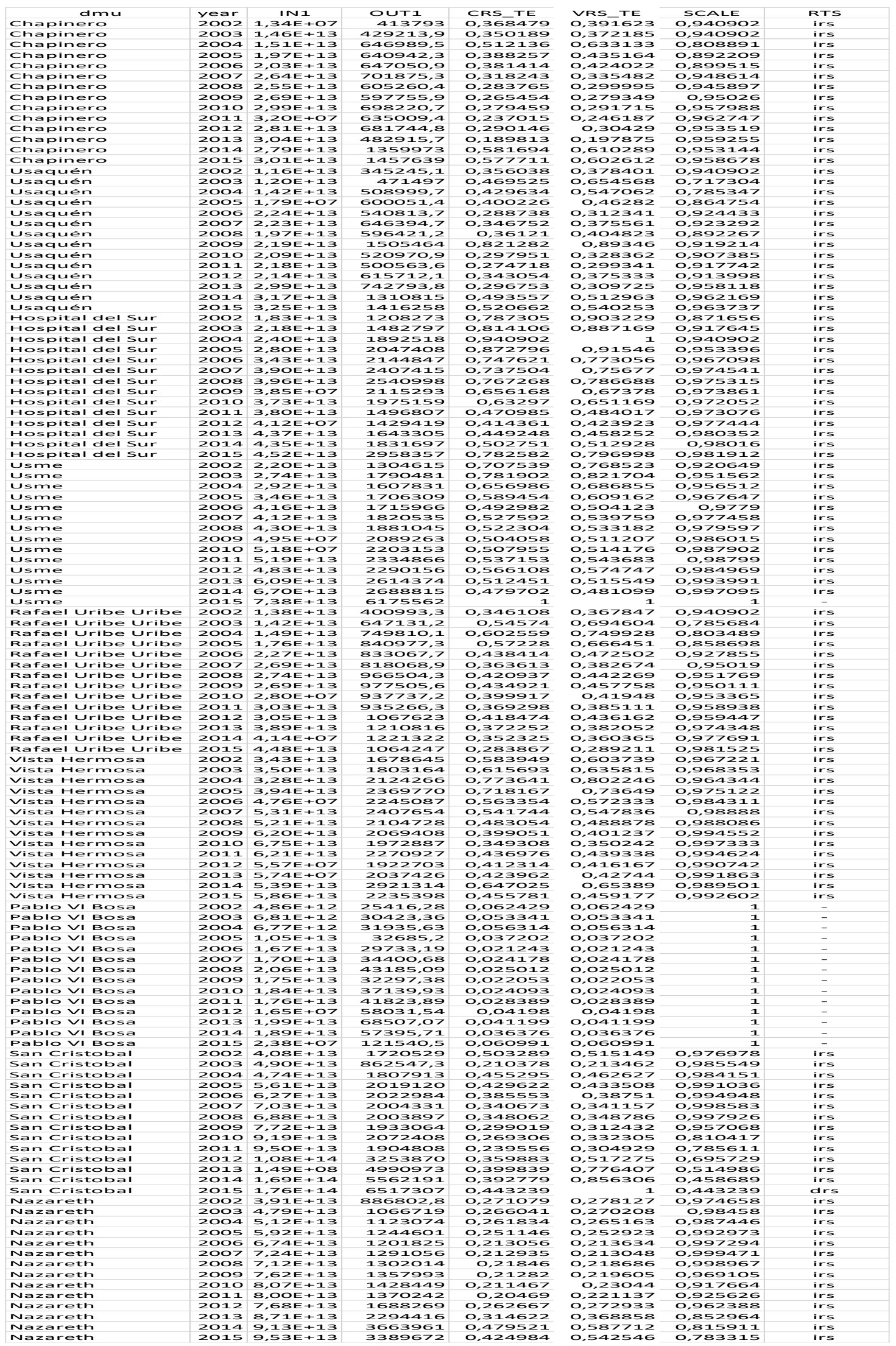




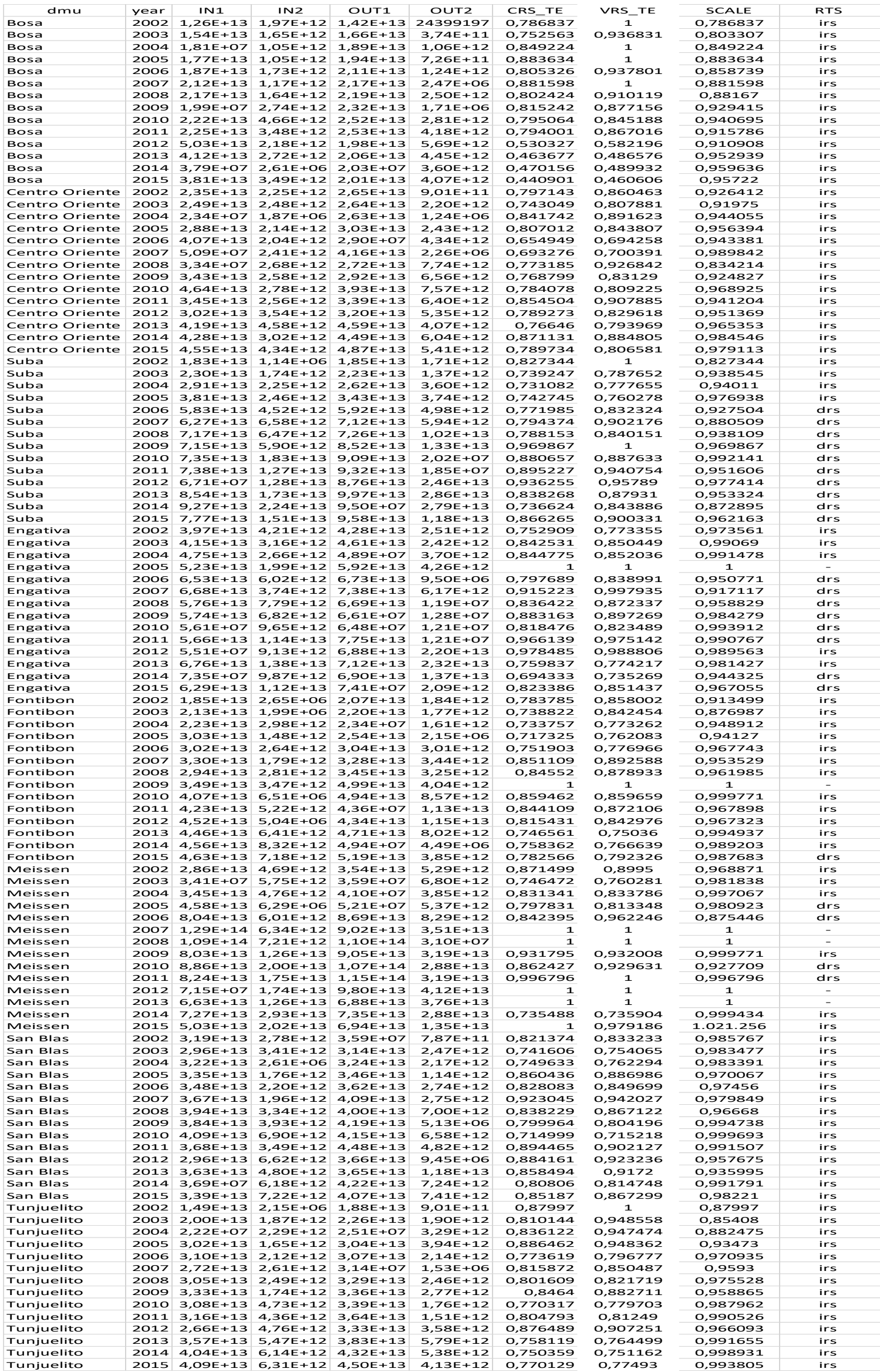


Anexo 4 Salida perspectiva científica II Nivel

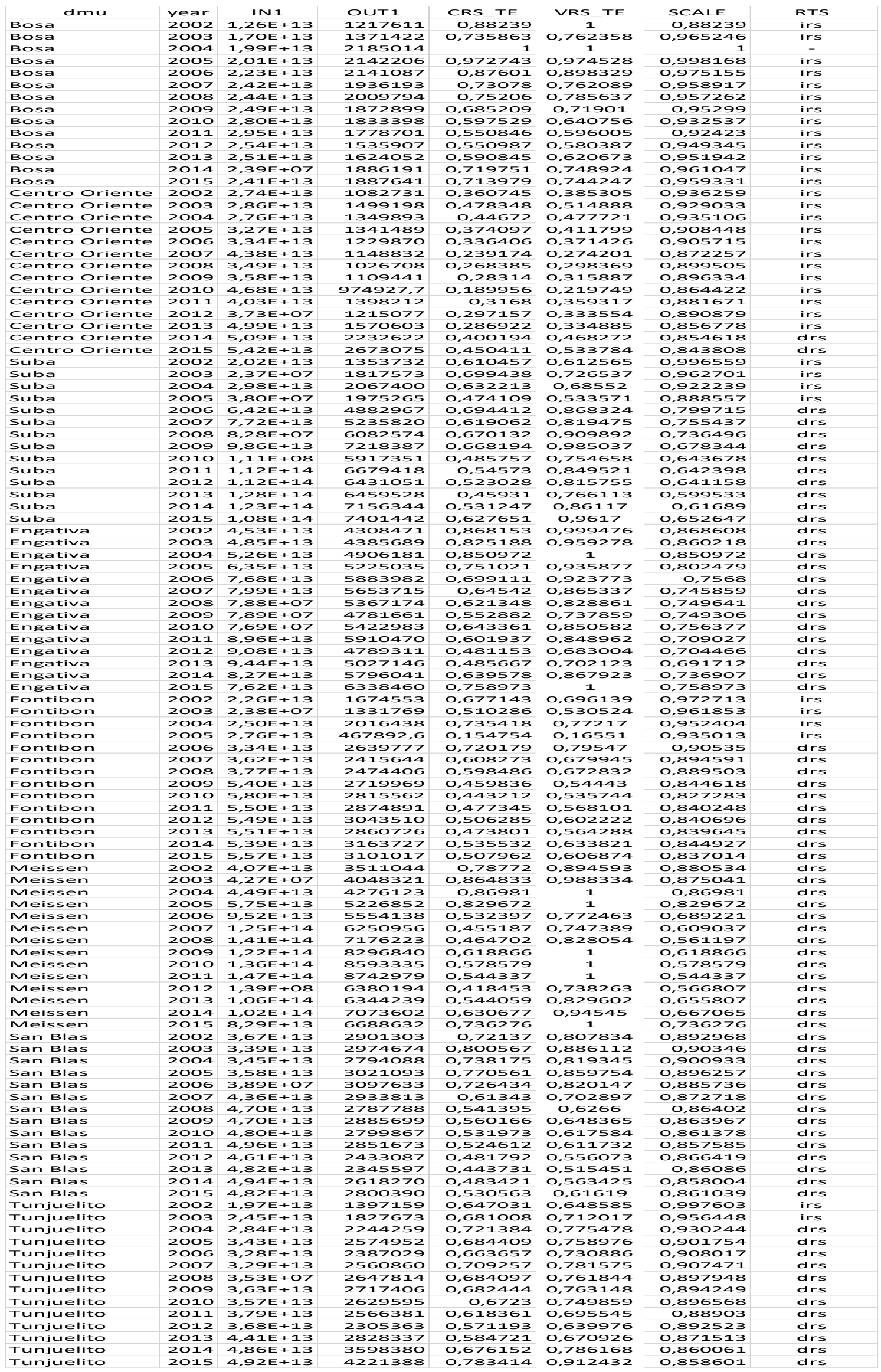




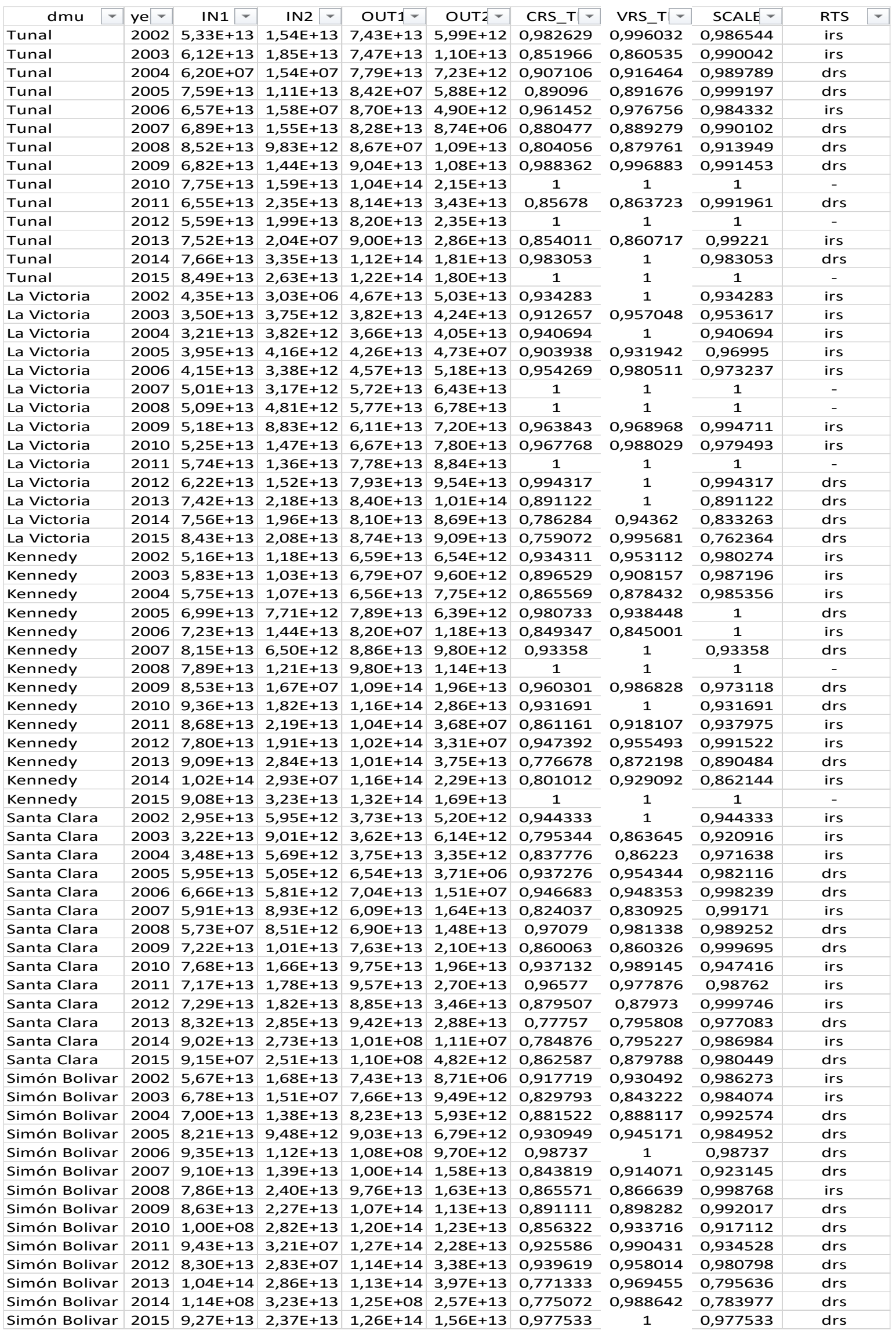




\begin{tabular}{|c|c|c|c|c|c|c|c|}
\hline $\mathrm{dmu}$ & year & IN1 & OUT1 & CRS_TE & VRS_TE & SCALE & RTS \\
\hline Tunal & 2002 & $8,03 E+13$ & 14172027 & 1 & 1 & 1 & - \\
\hline Tunal & 2003 & $8,58 E+13$ & 13763587 & 0,909599 & 0,937353 & 0,97039 & irs \\
\hline Tunal & 2004 & $8,51 E+07$ & 12231054 & 0,814572 & 0,83651 & 0,973775 & irs \\
\hline Tunal & 2005 & $9,01 E+13$ & 13093180 & 0,823753 & 0,867662 & 0,949395 & irs \\
\hline Tunal & 2006 & $9,19 E+13$ & 13104455 & 0,808242 & 0,858733 & 0,941203 & irs \\
\hline Tunal & 2007 & $9,15 E+13$ & 13337221 & 0,825935 & 0,875991 & 0,942859 & irs \\
\hline Tunal & 2008 & $9,76 E+07$ & 13701574 & 0,795576 & 0,867305 & 0,917298 & irs \\
\hline Tunal & 2009 & $1,01 E+14$ & 14249327 & 0,797762 & 0,882928 & 0,903542 & drs \\
\hline Tunal & 2010 & $1,26 \mathrm{E}+14$ & 13511138 & 0,609124 & 0,732659 & 0,831388 & irs \\
\hline Tunal & 2011 & $1,16 E+14$ & 13639010 & 0,668326 & 0,779556 & 0,857317 & irs \\
\hline Tunal & 2012 & $1,06 E+14$ & 12632500 & 0,678472 & 0,763642 & 0,88847 & irs \\
\hline Tunal & 2013 & $1,19 E+14$ & 14009250 & 0,669557 & 0,788337 & 0,849328 & irs \\
\hline Tunal & 2014 & $1,30 E+14$ & 14727411 & 0,643645 & 0,782774 & 0,822261 & drs \\
\hline Tunal & 2015 & $1,40 E+14$ & 17481717 & 0,706357 & 0,882469 & 0,800433 & drs \\
\hline La Victoria & 2002 & $9,70 E+13$ & 3601601 & 0,210353 & 0,228756 & 0,919554 & irs \\
\hline La Victoria & 2003 & $8,06 E+13$ & 4465852 & 0,31395 & 0,314494 & 0,998271 & irs \\
\hline La Victoria & 2004 & $7,71 E+07$ & 4723627 & 0,347181 & 0,348532 & 0,996126 & irs \\
\hline La Victoria & 2005 & $8,99 E+13$ & 5047612 & 0,318262 & 0,334906 & 0,950303 & irs \\
\hline La Victoria & 2006 & $9,75 E+13$ & 5568444 & 0,32379 & 0,352772 & 0,917845 & irs \\
\hline La Victoria & 2007 & $1,22 \mathrm{E}+14$ & 8300451 & 0,387302 & 0,460074 & 0,841826 & irs \\
\hline La Victoria & 2008 & $1,25 E+14$ & 8712370 & 0,393611 & 0,473079 & 0,832019 & irs \\
\hline La Victoria & 2009 & $1,33 E+08$ & 9354146 & 0,398394 & 0,488893 & 0,81489 & irs \\
\hline La Victoria & 2010 & $1,45 E+14$ & 9986484 & 0,39121 & 0,501989 & 0,77932 & irs \\
\hline La Victoria & 2011 & $1,66 E+14$ & 10758711 & 0,366825 & 0,540806 & 0,678293 & irs \\
\hline La Victoria & 2012 & $1,75 E+14$ & 11390944 & 0,369583 & 0,572587 & 0,645461 & irs \\
\hline La Victoria & 2013 & $1,85 E+14$ & 11936239 & 0,365179 & 0,599997 & 0,608634 & irs \\
\hline La Victoria & 2014 & $1,68 E+14$ & 12150102 & 0,409985 & 0,610747 & 0,671283 & irs \\
\hline La Victoria & 2015 & $1,78 E+14$ & 12343617 & 0,392259 & 0,620475 & 0,632192 & irs \\
\hline Kennedy & 2002 & $7,24 \mathrm{E}+07$ & 9045498 & 0,708027 & 0,715303 & 0,989827 & irs \\
\hline Kennedy & 2003 & $7,75 E+13$ & 9543104 & 0,697849 & 0,700217 & 0,996617 & irs \\
\hline Kennedy & 2004 & $7,33 E+13$ & 5675996 & 0,438839 & 0,442783 & 0,991092 & irs \\
\hline Kennedy & 2005 & $8,53 E+13$ & 12975266 & 0,861771 & 0,886077 & 0,972568 & irs \\
\hline Kennedy & 2006 & $9,38 E+13$ & 14433450 & 0,872383 & 0,935001 & 0,933029 & drs \\
\hline Kennedy & 2007 & $9,84 E+13$ & 14435049 & 0,83115 & 0,909274 & 0,914081 & drs \\
\hline Kennedy & 2008 & $1,09 E+14$ & 15686943 & 0,812885 & 0,927986 & 0,875967 & drs \\
\hline Kennedy & 2009 & $1,28 E+14$ & 17266187 & 0,762377 & 0,923823 & 0,825242 & drs \\
\hline Kennedy & 2010 & $1,45 E+14$ & 16236993 & 0,636695 & 0,816182 & 0,78009 & drs \\
\hline Kennedy & 2011 & $1,4 \mathrm{OE}+14$ & 15211884 & 0,614433 & 0,767714 & 0,800341 & drs \\
\hline Kennedy & 2012 & $1,35 E+14$ & 15374703 & 0,645421 & 0,795982 & 0,810849 & drs \\
\hline Kennedy & 2013 & $1,39 E+14$ & 17861220 & 0,730047 & 0,908552 & 0,803529 & drs \\
\hline Kennedy & 2014 & $1,39 E+14$ & 17743562 & 0,724485 & 0,901945 & 0,803248 & drs \\
\hline Kennedy & 2015 & $1,49 E+14$ & 17036399 & 0,649596 & 0,856366 & 0,758549 & drs \\
\hline Santa Clara & 2002 & $4,25 E+13$ & 4099153 & 0,54703 & 0,59651 & 0,917051 & irs \\
\hline Santa Clara & 2003 & $4,23 E+13$ & 4956064 & 0,663964 & 0,724564 & 0,916364 & irs \\
\hline Santa Clara & 2004 & $4,09 E+13$ & 6560566 & 0,910096 & 1 & 0,910096 & irs \\
\hline Santa Clara & 2005 & $6,91 E+13$ & 9002558 & 0,738697 & 0,750067 & 0,984841 & irs \\
\hline Santa Clara & 2006 & $8,55 E+13$ & 8814701 & 0,58434 & 0,601333 & 0,971742 & irs \\
\hline Santa Clara & 2007 & $7,73 E+13$ & 8669979 & 0,635699 & 0,638022 & 0,996359 & irs \\
\hline Santa Clara & 2008 & $8,38 E+13$ & 8833868 & 0,597743 & 0,609425 & 0,98083 & irs \\
\hline Santa Clara & 2009 & $9,74 E+13$ & 9638392 & 0,561106 & 0,611028 & 0,918297 & irs \\
\hline Santa Clara & 2010 & $1,17 E+14$ & 10761147 & 0,520569 & 0,610156 & 0,853172 & irs \\
\hline Santa Clara & 2011 & $1,23 E+14$ & 10979600 & 0,507068 & 0,604613 & 0,838666 & irs \\
\hline Santa Clara & 2012 & $1,23 E+08$ & 11397202 & 0,524688 & 0,626345 & 0,837698 & irs \\
\hline Santa Clara & 2013 & $1,23 E+14$ & 13228766 & 0,609635 & 0,727477 & 0,838012 & irs \\
\hline Santa Clara & 2014 & $1,12 \mathrm{E}+14$ & 12981234 & 0,655699 & 0,756014 & 0,867311 & irs \\
\hline Santa Clara & 2015 & $1,15 E+14$ & 14737221 & 0,727545 & 0,846235 & 0,859744 & drs \\
\hline Simón Bolivar & 2002 & $8,30 E+13$ & 12183588 & 0,832274 & 0,844857 & 0,985107 & irs \\
\hline Simón Bolivar & 2003 & $8,60 E+13$ & 12823303 & 0,844672 & 0,871737 & 0,968953 & irs \\
\hline Simón Bolivar & 2004 & $8,82 E+13$ & 12347670 & 0,79328 & 0,827886 & 0,958201 & irs \\
\hline Simón Bolivar & 2005 & $9,71 E+13$ & 12878926 & 0,751509 & 0,81757 & 0,919198 & irs \\
\hline Simón Bolivar & 2006 & $1,18 E+14$ & 13173739 & 0,633662 & 0,744297 & 0,851356 & irs \\
\hline Simón Bolivar & 2007 & $1,16 E+14$ & 13648844 & 0,666641 & 0,778544 & 0,856266 & irs \\
\hline Simón Bolivar & 2008 & $1,14 \mathrm{E}+14$ & 13476617 & 0,670476 & 0,777562 & 0,86228 & irs \\
\hline Simón Bolivar & 2009 & $1,18 E+08$ & 13466457 & 0,646218 & 0,759713 & 0,850607 & irs \\
\hline Simón Bolivar & 2010 & $1,32 E+14$ & 13989307 & 0,60179 & 0,735936 & 0,817721 & irs \\
\hline Simón Bolivar & 2011 & $1,50 E+14$ & 15571353 & 0,59014 & 0,782723 & 0,753958 & drs \\
\hline Simón Bolivar & 2012 & $1,47 E+14$ & 14478669 & 0,556745 & 0,727797 & 0,764973 & drs \\
\hline Simón Bolivar & 2013 & $1,52 E+08$ & 13842487 & 0,515759 & 0,695818 & 0,741227 & irs \\
\hline Simón Bolivar & 2014 & $1,51 \mathrm{E}+14$ & 14305355 & 0,537465 & 0,719085 & 0,747429 & drs \\
\hline Simón Bolivar & 2015 & $1,41 E+14$ & 19893829 & 0,798744 & 1 & 0,798744 & drs \\
\hline
\end{tabular}

